\title{
$\alpha$-Alkylation of Ketones with Alkenes Enabled by Photo- Induced Activation of Silyl enol ethers in the Presence of a Small Amount of Water
}

\author{
Tsubasa Hirata, Yoshihiro Ogasawara, Yasuhiro Yamashita*, Shū Kobayashi* \\ Department of Chemistry, School of Science, The University of Tokyo, Hongo, Bunkyo-ku, Tokyo, Japan,
} 113-0033

\section{Supporting Information}

Table of contents

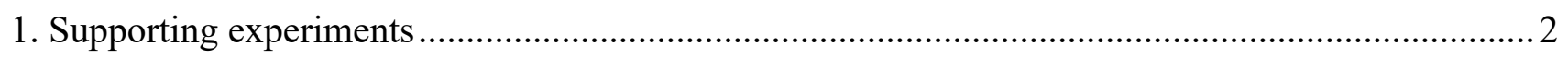

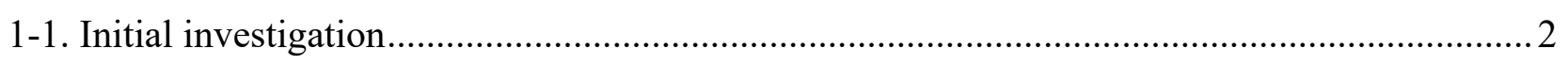

1-2. Optimization (photocatalyst, additive, solvent) ….......................................................... 3



1-4. Comparison of the reactivities between $(Z)$ - and $(E)$-isomers ........................................... 5

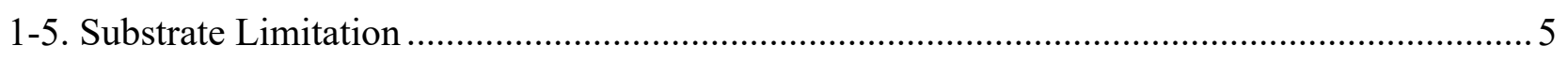

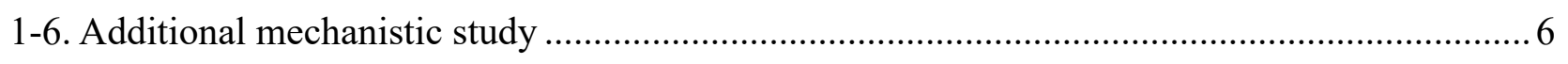

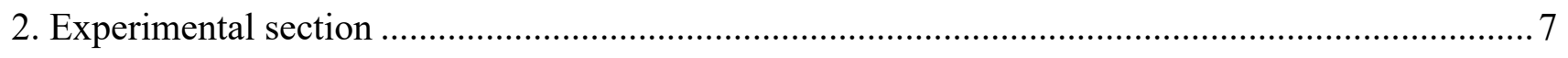

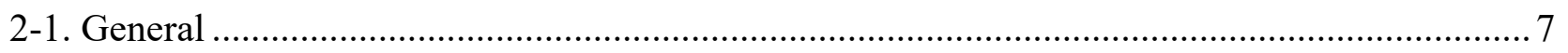

2-2. Photoreactor system utilized in described research...................................................... 8

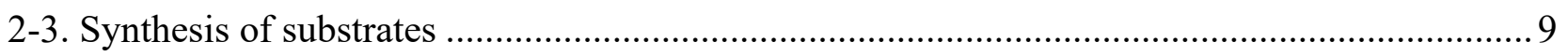





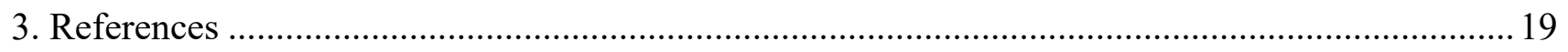






\section{Supporting experiments \\ 1-1. Initial investigation}

Initial investigation was conducted to find appropriate photocatalysts for the desired reaction (Table S1). We first chose DCA for the photocatalyst because it was known that photo-activated DCA oxidized silyl enol ethers to afford the corresponding radical cations. However, side-adduct $\mathbf{4}$ derived from employed alkene 1a and methanol was obtained without formation of the desired adduct 3aa (entry 1). Similarly, DCN afforded the side-adduct 4 under UV irradiation (entry 2). Judging from reduction potentials of them, the side-reaction was caused by oxidation of the alkene 1a. To suppress the undesired oxidation, the photocatalyst was change to 4CzIPN, which has lower reduction potential. Consequently, the desired adduct 3aa was obtained without formation of the side-adduct 4 .

Table S1. Effect of photocatalyst structures

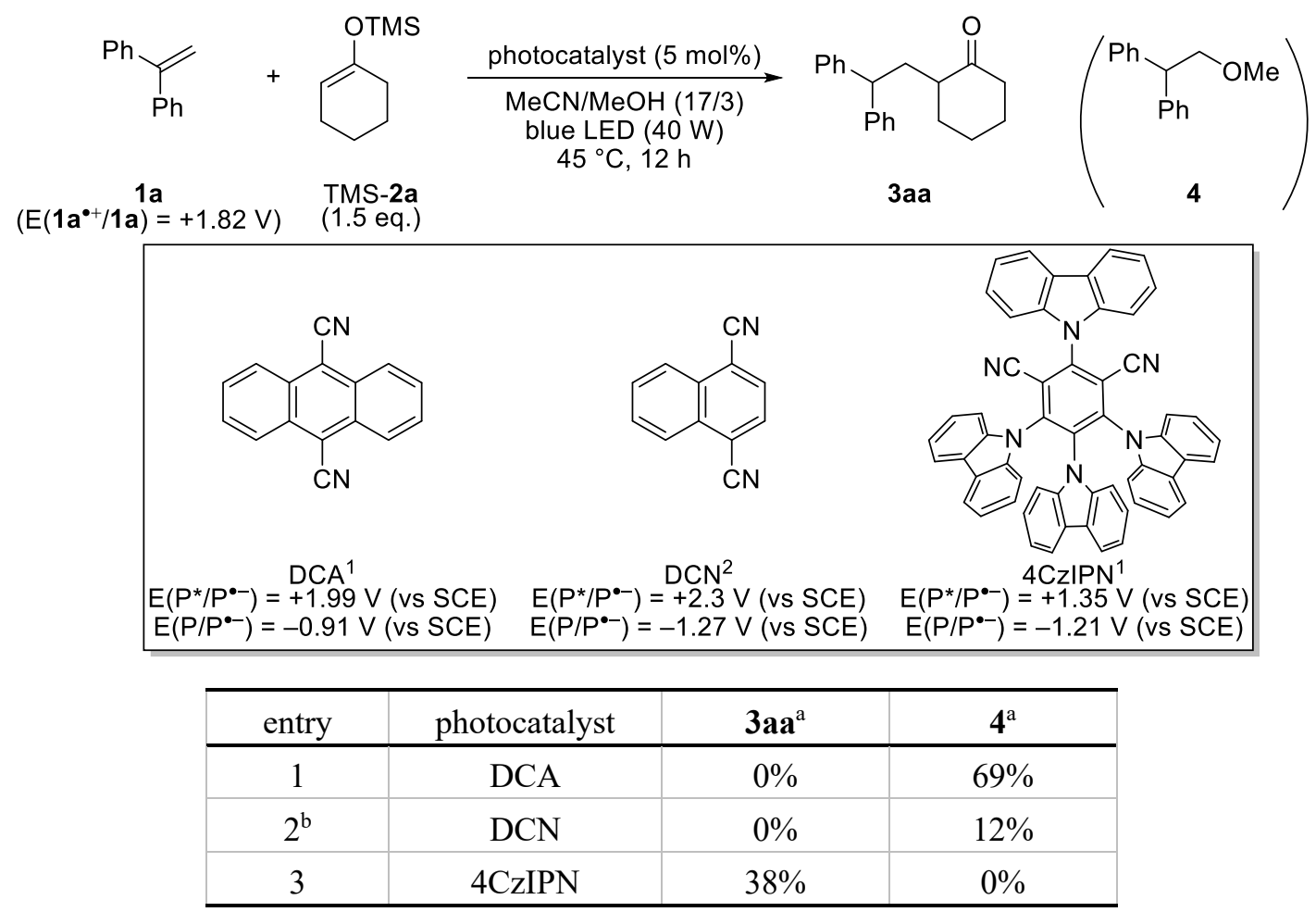

a The yields were determined by ${ }^{1} \mathrm{H}$ NMR analysis (internal standard: $\mathrm{CH}_{2} \mathrm{Br}_{2}$ ). ${ }^{\mathrm{b}}$ UV-light was used instead of blue LED. 


\section{1-2. Optimization (photocatalyst, additive, solvent)}

As shown in the main manuscript, the reaction using 1.2 equivalents of water proceeded smoothly under blue light irradiation conditions (Table 1, entry 7). In this context, we found that the desired product was obtained in 77\% yield within 2 hours in the presence of only $1 \mathrm{~mol} \%$ of $4 \mathrm{CzIPN}$ (Table S2, entry 1). Photocatalysts, additives, and solvents were further investigated to realize more efficient reaction system. Although several photocatalysts such as 4DPAIPN or 4CzTPN were firstly tested, the desired product was not obtained at all (entries 2, 3). Next, methanol or isopropyl alcohol was examined as an additive instead of water, but the desired product 3aa was obtained in lower yields (entries 4, 5). Although a variety of solvents were also tested, the yields were not improved (entries 69). We next prolonged the reaction time up to 6 hours, and the yield was improved up to $86 \%$ (entry 10). Eventually, the reaction proceeded in excellent yield within 2 hours in the presence of $2 \mathrm{~mol} \%$ of the photocatalyst 4CzIPN (entry 11).

Table S2. Optimization of reactions conditions
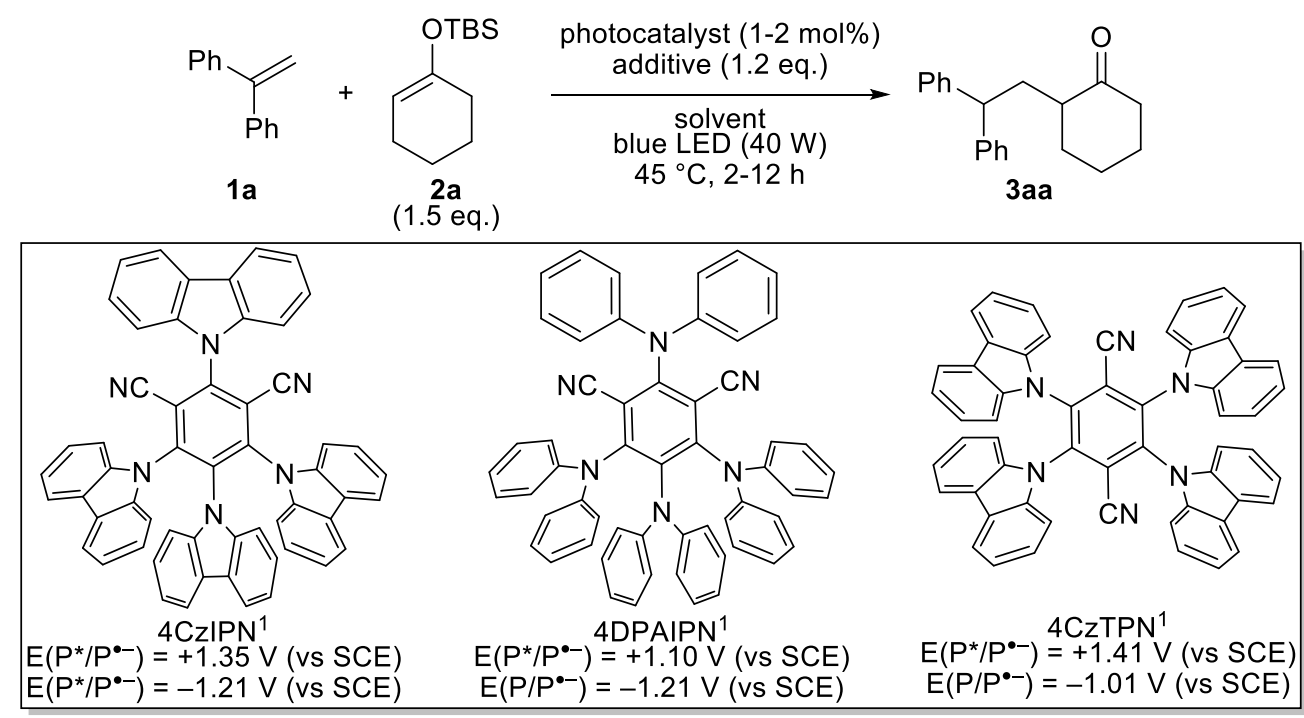

\begin{tabular}{|c|c|c|c|c|c|}
\hline entry & photocatalyst & additive & time $(\mathrm{h})$ & solvent & yield $(\%)^{\mathrm{a}}$ \\
\hline 1 & 4CzIPN (1 mol\%) & $\mathrm{H}_{2} \mathrm{O}$ (1.2 eq.) & 2 & $\mathrm{MeCN}$ & 77 \\
\hline 2 & 4DPAIPN (1 mol\%) & $\mathrm{H}_{2} \mathrm{O}$ (1.2 eq.) & 2 & $\mathrm{MeCN}$ & 0 \\
\hline 3 & 4CzTPN (1 mol\%) & $\mathrm{H}_{2} \mathrm{O}$ (1.2 eq.) & 2 & $\mathrm{MeCN}$ & 0 \\
\hline 4 & 4CzIPN (1 mol\%) & $\mathrm{MeOH}$ (1.2 eq.) & 2 & $\mathrm{MeCN}$ & 73 \\
\hline 5 & 4CzIPN (1 mol\%) & ${ }^{i} \mathrm{PrOH}$ (1.2 eq.) & 2 & $\mathrm{MeCN}$ & 67 \\
\hline 6 & 4CzIPN (1 mol\%) & $\mathrm{H}_{2} \mathrm{O}$ (1.2 eq.) & 2 & $\mathrm{MeOH}$ & 0 \\
\hline 7 & 4CzIPN (1 mol\%) & $\mathrm{H}_{2} \mathrm{O}$ (1.2 eq.) & 2 & THF & 0 \\
\hline 8 & 4CzIPN (1 mol\%) & $\mathrm{H}_{2} \mathrm{O}$ (1.2 eq.) & 2 & acetone & 25 \\
\hline 9 & 4CzIPN (1 mol\%) & $\mathrm{H}_{2} \mathrm{O}$ (1.2 eq.) & 2 & DMF & 13 \\
\hline 10 & 4CzIPN (1 mol\%) & $\mathrm{H}_{2} \mathrm{O}$ (1.2 eq.) & 6 & $\mathrm{MeCN}$ & 86 \\
\hline 11 & 4CzIPN (2 mol\%) & $\mathrm{H}_{2} \mathrm{O}$ (1.2 eq.) & 2 & $\mathrm{MeCN}$ & $92(90)^{\mathrm{b}}$ \\
\hline
\end{tabular}

a The yields were determined by ${ }^{1} \mathrm{H}$ NMR analysis (internal standard: $\mathrm{CH}_{2} \mathrm{Br}_{2}$ ). ${ }^{\mathrm{b}}$ The yield shown in parentheses is based on the isolated product. 


\section{1-3. Optimization for styrene}

For expansion of the substrate scope to styrene derivatives, the reaction of styrene was further optimized (Table S3). First, the desired product was not obtained at all although the reaction was carried out under the optimized conditions (entry 1). Reduction of radical B by the reduced photocatalyst $\left(4 \mathrm{CzIPN}^{-*}\right.$ ) seemed to be more difficult than that of radical A judging from the reduction potentials. To address this issue, benzenethiol $(\mathrm{PhSH})$ was added as a HAT catalyst, and the desired product 4 ha was obtained in moderate yield (entry 2). By using $5 \mathrm{~mol} \%$ of the photocatalyst, the reaction proceeded in good yield (entry 3). Next, several thiols were examined, but the yield was not improved eventually (entries 4, 5). Finally, the desired product was obtained in high yield by using 50 mol\% of PhSH. On the other hand, the conditions shown in entry 3 were chosen as the standard conditions in the substrate scope investigation because $50 \mathrm{~mol} \%$ of $\mathrm{PhSH}$ seemed to be too much amount. But, this result implies that the yields can be improved if necessary.

Table S3. Optimization of HAT catalyst structures

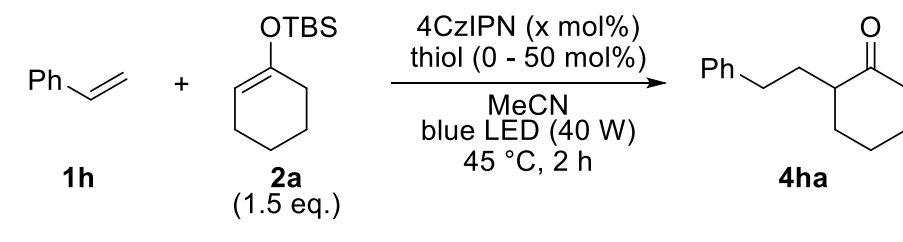

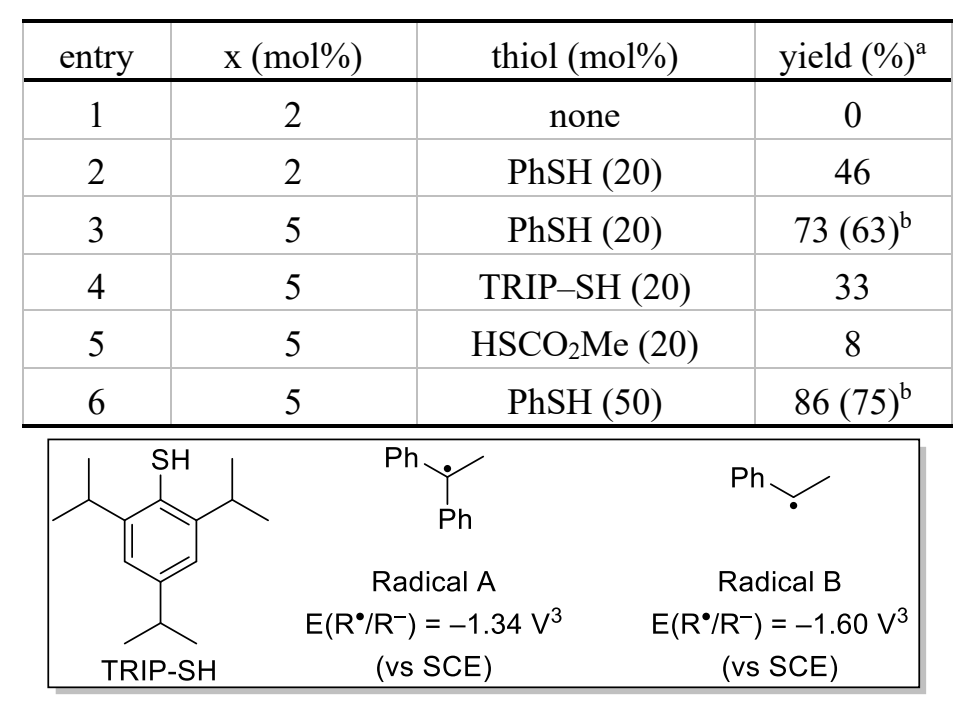

a The yields were determined by ${ }^{1} \mathrm{H}$ NMR analysis (internal standard: $\mathrm{CH}_{2} \mathrm{Br}_{2}$ ). ${ }^{\mathrm{b}}$ The yields shown in parentheses are based on the isolated product. 


\section{1-4. Comparison of the reactivities between $(Z)$ - and $(E)$-isomers}

Silyl enol ethers sometimes have $(Z)$ - or $(E)$-forms. We were interested in the difference of their reactivities between those forms, and the reactivities were compared by using a mixture of the isomers (Scheme S1). When the (Z)-isomer was the main component, the reaction proceeded in good yield. On the other hand, the desired product was obtained in moderate yield when the main component was the $(E)$-isomer. Those results implied that $(Z)$-isomer was more reactive than the other. But, the yield of (E)-isomer was not so low, and it seemed to be employable as a starting material.

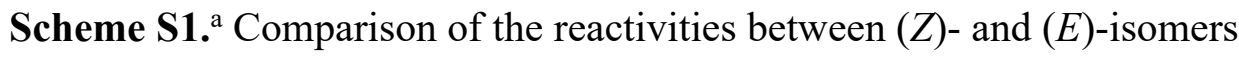<smiles>[CH2+]CC(=C[CH+]C=C(c1ccccc1)c1ccccc1)OC</smiles>

$1 \mathrm{a}$

(Z)-TMS-2f<smiles>C/C=C(/CC)O[Na]</smiles>

(E)-TMS-2f

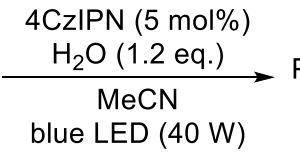

$45^{\circ} \mathrm{C}, 12 \mathrm{~h}$

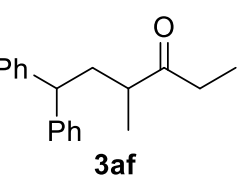

$Z / E=90 / 10: 73 \%$ yield $Z / E=33 / 67: 55 \%$ yield

a The yields were determined by ${ }^{1} \mathrm{H}$ NMR analysis (internal standard: $\mathrm{CH}_{2} \mathrm{Br}_{2}$ ).

\section{1-5. Substrate Limitation}

Silyl enol ethers derived deriving from methyl ketones, such as acetophenone and tert-butyl methyl ketone, were tested (Scheme S2). But, the reactions did not proceed at all. We assumed that it was caused by instability of primally $\alpha$-carbonyl radicals.

Scheme S2. Substrate Limitation
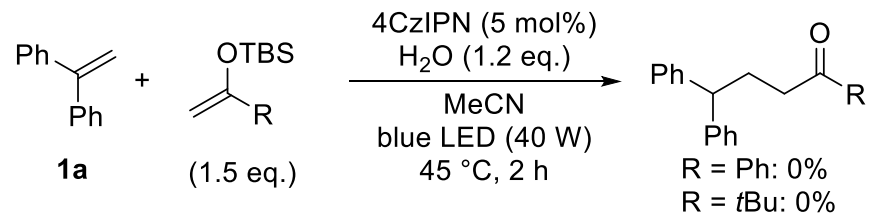


\section{1-6. Additional mechanistic study}

To get an insight of the reaction mechanism, a radical trapping experiment was conducted (Scheme S3). The reaction was carried out in the presence of TEMPO, and the desired product 3aa was not obtained at all. Instead, 2a-OTMP was obtained, ${ }^{4}$ which could be formed by the reaction of the generated $\alpha$-carbonyl radical with TEMPO. This result suggested that the reactions proceeded in radical addition mechanism.

Scheme S3. Radical trapping experiment

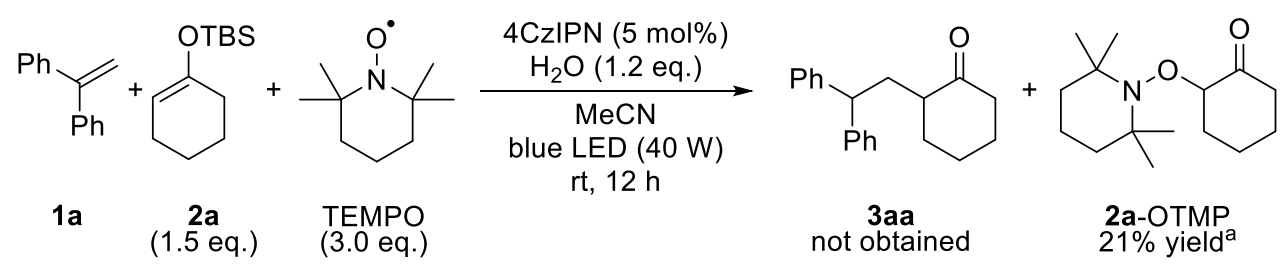

${ }^{a}$ The yield was determined by ${ }^{1} \mathrm{H}$ NMR analysis based on 2a (internal standard: 1,3,5trimethoxybenzene, $12.8 \mathrm{mg}$ ).

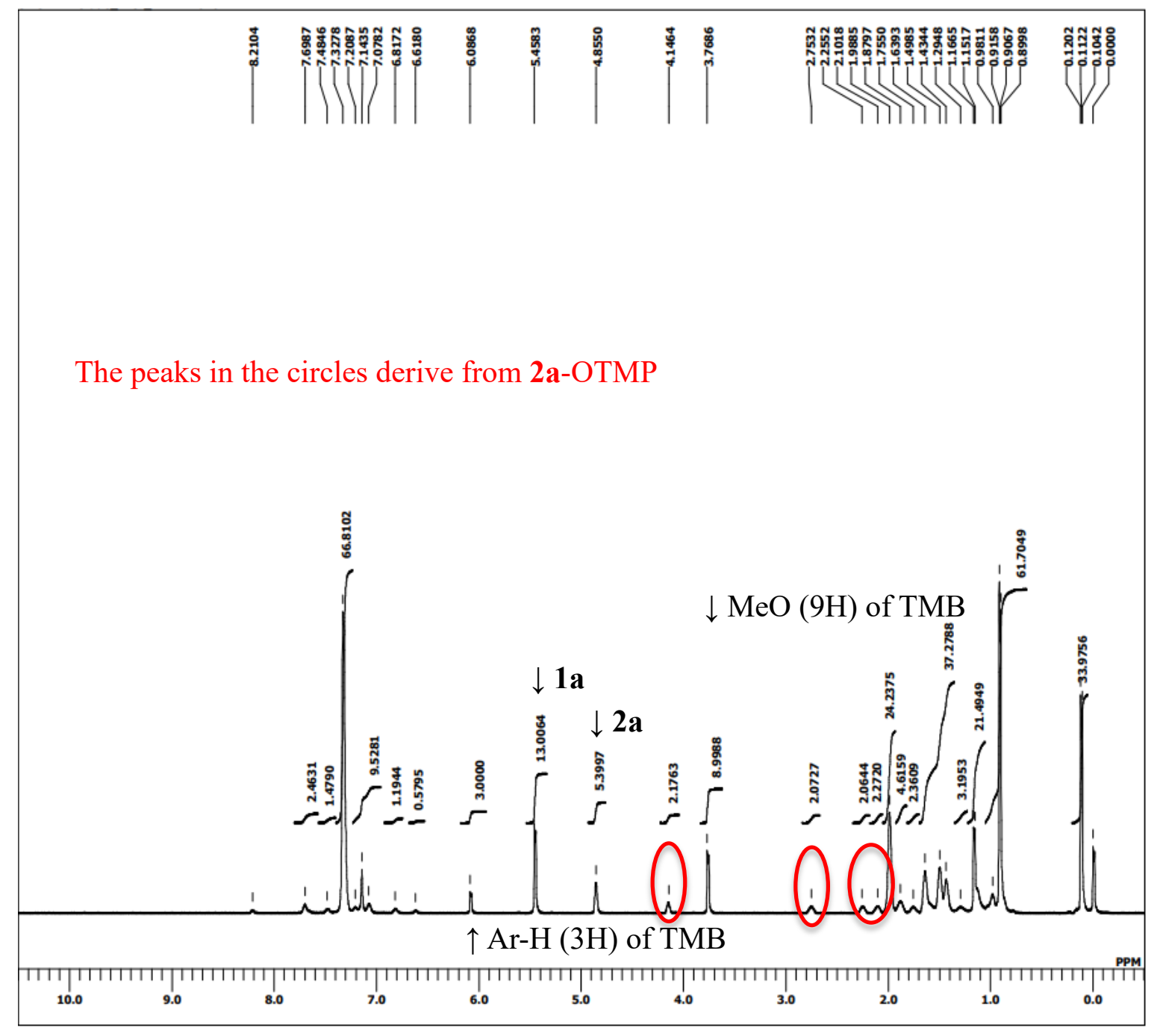

${ }^{1} \mathrm{H}$ NMR of the crude sample 


\section{Experimental section}

\section{2-1. General}

Melting points were measured with AS ONE Melting Temperature Mesurement Device (ATM02). ${ }^{1} \mathrm{H},{ }^{13} \mathrm{C}$, and ${ }^{19} \mathrm{FNMR}$ spectra were recorded on JEOL JNM-ECA500 and JNM-ECX600 spectrometers in $\mathrm{CDCl}_{3}$ unless otherwise noted. Tetramethylsilane (TMS) served as internal standard $(\delta=0)$ for ${ }^{1} \mathrm{H} \mathrm{NMR}$, and $\mathrm{CDCl}_{3}$ served as internal standard $(\delta=77.0)$ for ${ }^{13} \mathrm{C}$ NMR. Benzotrifluoride (BTF) served as internal standard $(\delta=-63.72)$ for ${ }^{19} \mathrm{~F}$ NMR. IR spectra were measured on JASCO FT/IR-610 spectrometer. DART mass spectra (TOF) were recorded on JEOL JMS-T100TD mass spectrometer. X-ray crystal structures were analyzed on a Rigaku R-AXIS RAPID diffractometer using multi-layer mirror monochromated Mo-K $\alpha$ radiation. Preparative thin-layer chromatography (PTLC) was carried out using Wakogel B-5F. Kessil LED Aquarium Light A160WE Tuna Blue was used as a blue light source. 200W Hg lamp was employed as UV-light source, and the reactions under UV irradiation were carried out in quartz tube. Alkenes $\left(1 \mathrm{a},{ }^{5} 1 \mathrm{~b},{ }^{5} 1 \mathrm{c},{ }^{6} 1 \mathrm{~d},{ }^{5} 1 \mathrm{e},{ }^{5} 1 \mathrm{f},{ }^{5} 1 \mathrm{~g},{ }^{5} 1 \mathrm{k},{ }^{7} 1 \mathrm{l},{ }^{5} 1 \mathrm{~m},{ }^{5} 1 \mathrm{n},{ }^{8}\right.$ $\mathbf{1 p},{ }^{5} \mathbf{1 q},{ }^{5} \mathbf{1} \mathbf{r}^{8}$ ), silyl enol ethers $\left(\mathbf{2 a},{ }^{9} \mathbf{2 b},{ }^{9} \mathbf{2 c},{ }^{9} \mathbf{2 d},{ }^{10} \mathbf{2} \mathbf{f},{ }^{11,12,13} \mathbf{2} \mathbf{g}^{11}\right.$ ), photocatalysts (DCA, ${ }^{14}$ DCN, ${ }^{15}$ $4 \mathrm{CzIPN},{ }^{2} 4 \mathrm{DPAIPN},{ }^{2} 4 \mathrm{CzTPN}^{2}$ ) were synthesized according to the cited literatures. The other starting materials were commercially available and used after distillation. 


\section{2-2. Photoreactor system utilized in described research}

The reaction tubes were irradiated by Kessil LED Aquarium Light A160WE Tuna Blue (highest blue and intensity setting, The distance between the tubes and the LED: $\sim 2 \mathrm{~cm}$ ). A simple cooling fan was equipped for cooling. It was measured that the equilibrium temperature of the reaction mixture was $45^{\circ} \mathrm{C}$ with a standard alcohol thermometer.

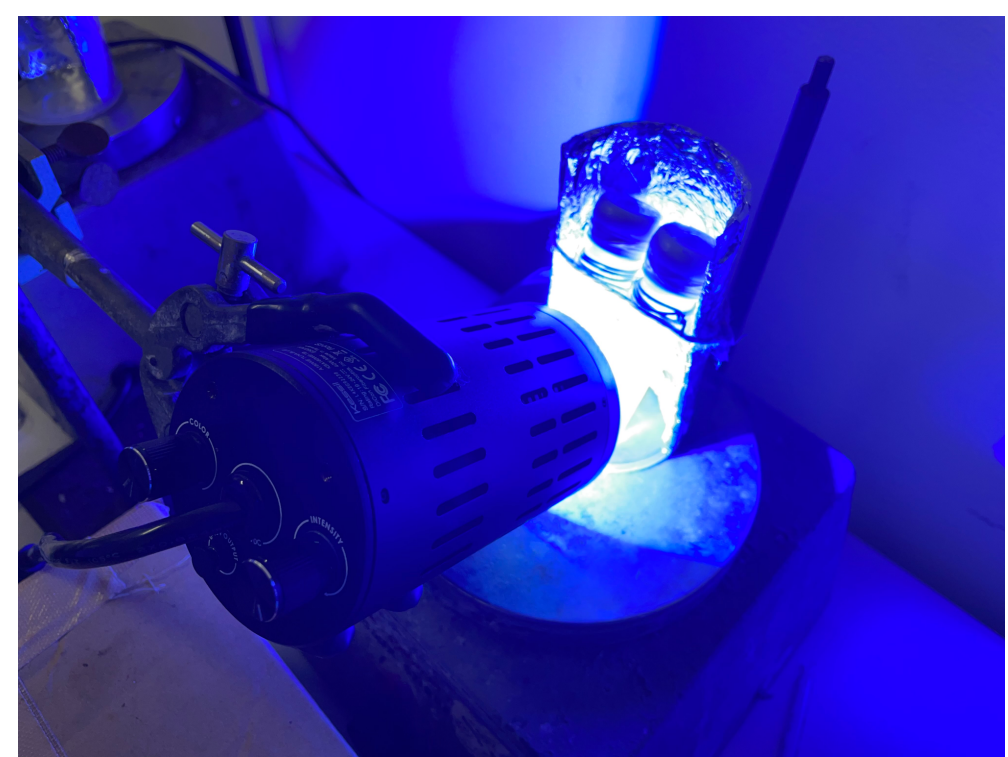

Figure S1. Photo-reactor



Figure S2. The outside appearance of the photo-reactor 


\section{2-3. Synthesis of substrates}

Synthesis of tert-butyldimethyl((3-methylcyclohex-1-en-1-yl)oxy)silane (2e)

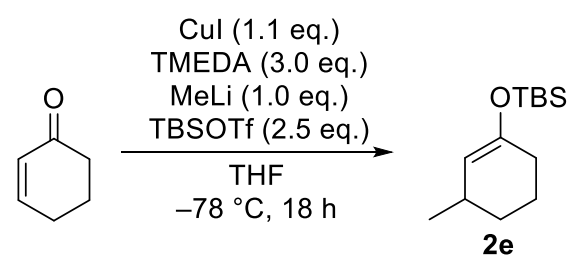

The title compound (2e) was synthesized according to a literature. ${ }^{16}$ Copper (I) iodide $(6.29 \mathrm{~g}$, $33.0 \mathrm{mmol})$ and TMEDA $(13.5 \mathrm{~mL}, 90.0 \mathrm{mmol})$ were added to a clean dry, three-necked $300 \mathrm{~mL}$ flask equipped with a magnetic stirrer and an addition funnel. The apparatus was then flushed with argon and $50 \mathrm{~mL}$ of dry THF was added. After the contents were stirred at room temperature for 30 minutes, the flask was cooled to $-78{ }^{\circ} \mathrm{C}$ and methyl lithium (3.1 $\mathrm{M}$ in diethoxymethane, $9.7 \mathrm{~mL}, 30.0 \mathrm{mmol}$ ) was added followed by stirring at $-78{ }^{\circ} \mathrm{C}$ for $20 \mathrm{~min}$. Then, cyclohex-2-enone $(2.88 \mathrm{~g}, 30.0 \mathrm{mmol})$ in $20 \mathrm{~mL}$ of dry THF was added dropwise for 30 minutes followed by tert-butyldimethylsilyl trifluoromethanesulfonate $(17.2 \mathrm{~mL}, 75.0 \mathrm{mmol})$ in $20 \mathrm{~mL}$ of dry THF was added, and the reaction mixture was stirred at $-78{ }^{\circ} \mathrm{C}$ for 18 hours. The reaction was quenched by saturated $\mathrm{NaHCO}_{3}$ aqueous solution and the obtained mixture was extracted with hexane $(100 \mathrm{~mL} \times 3)$. The combined organic layer was washed with brine $(\times 3)$ and dried over anhydrous $\mathrm{Na}_{2} \mathrm{SO}_{4}$. After filtration and concentration under reduced pressure, the obtained crude product was purified by short column chromatography (hexane/Et $\left.{ }_{3} \mathrm{~N}=95 / 5\right)$ on silica gel. Then, the residue was distilled to afford the silyl enol ether $2 \mathrm{e}$ (3.08 g, $45 \%$ yield).

tert-butyldimethyl((3-methylcyclohex-1-en-1-yl)oxy)silane (2e); Colorless liquid; ${ }^{1} \mathrm{H}$ NMR (500 $\left.\mathrm{MHz} \mathrm{CDCl}_{3}\right) \delta: 4.74(\mathrm{~s}, 1 \mathrm{H}), 2.24-2.22(\mathrm{~m}, 1 \mathrm{H}), 2.03-1.91(\mathrm{~m}, 2 \mathrm{H}), 1.76-1.74(\mathrm{~m}, 1 \mathrm{H}), 1.70-1.67(\mathrm{~m}$, $1 \mathrm{H}), 1.57-1.53(\mathrm{~m}, 1 \mathrm{H}), 1.08-1.01(\mathrm{~m}, 1 \mathrm{H}), 0.94(\mathrm{~d}, 3 \mathrm{H}, J=6.80 \mathrm{~Hz}), 0.92(\mathrm{~s}, 9 \mathrm{H}), 0.12(\mathrm{~s}, 6 \mathrm{H}) ;{ }^{13} \mathrm{C}$ NMR (150 MHz, $\left.\mathrm{CDCl}_{3}\right) \delta: 150.2,111.1,31.2,29.8,29.5,25.7,22.5,21.8,18.0,-4.4,-4.5$; IR (neat, $\mathrm{cm}^{-1}$ ); 2954, 2929, 2859, 1194, 1181; HRMS (DART) calcd for $\mathrm{C}_{13} \mathrm{H}_{27} \mathrm{OSi}[\mathrm{M}+\mathrm{H}]^{+} 227.1831$, found 227.1829. 


\section{2-4. Substrate scope}

\section{General procedure for the photo-induced reactions}



4CzIPN (7.9 mg, $10.0 \mu \mathrm{mol})$, alkene $(0.500 \mathrm{mmol})$, and silyl enol ether $(0.750 \mathrm{mmol})$ were placed in an $8 \mathrm{~mL}$ pyrex tube (Diameter: $14 \mathrm{~mm}$ ), and the tube was sealed with a septum. The tube was evacuated to remove air and refilled with argon via a needle. Acetonitrile $(5.0 \mathrm{~mL})$ and pure water $(10.8 \mu \mathrm{L})$ were then added. When benzenethiol was used, it $(10.2 \mu \mathrm{L}, 0.100 \mathrm{mmol})$ was added through the septum. A vent needle was attached, and the reaction mixture was degassed by sparging with argon under sonication for $15 \mathrm{~min}$. The reaction mixture was stirred at room temperature under blue LED irradiation for $12 \mathrm{~h}$. Subsequently, $1 \mathrm{~N}$ aq. $\mathrm{HCl}(1.0 \mathrm{~mL})$ was added, and the mixture was stirred for additional $30 \mathrm{~min}$. The mixture was basified with saturated $\mathrm{NaHCO}_{3}$ and extracted with dichloromethane $(10 \mathrm{~mL} \times 3)$. The combined organic layer was dried over anhydrous $\mathrm{Na}_{2} \mathrm{SO}_{4}$. After filtration and concentration under reduced pressure, the crude mixture was purified by PTLC to afford the desired product 3 .

In the double scale reaction (Table 1, entry 10 in the main manuscript), a $15 \mathrm{~mL}$ pyrex tube (Diameter: $14 \mathrm{~mm}$ ) was used as a reaction container. Following the general procedure, the reaction was carried out using 4CzIPN (15.8 mg, $20.0 \mu \mathrm{mol})$, 1a $(180.3 \mathrm{mg}, 1.000 \mathrm{mmol})$, silyl enol ether (318.6 mg, $1.500 \mathrm{mmol})$, Acetonitrile $(10.0 \mathrm{~mL})$, and pure water $(21.6 \mu \mathrm{L}, 1.20 \mathrm{mmol})$ were employed. The reaction container was placed between two light sources for more effective irradiation, and the mixture were stirred for $24 \mathrm{~h}$ under blue light irradiation. The desired product 3aa was obtained in $80 \%$ yield (221.4 mg, Table 1, entry 10$)$.

2-(2,2-Diphenylethyl)cyclohexan-1-one (3aa); $90 \%$ yield (131.6 mg, Table 1, entry 9); The product O was purified by PTLC (hexane/acetone =9/1); Colorless solid; Mp: 85-87 ${ }^{\circ} \mathrm{C}$; ${ }^{1} \mathrm{H}$ NMR $\left(600 \mathrm{MHz} \mathrm{CDCl}_{3}\right) \delta: 7.28-7.21(\mathrm{~m}, 8 \mathrm{H}), 7.18-7.15(\mathrm{~m}, 2 \mathrm{H}), 4.06(\mathrm{t}, 1 \mathrm{H}, J=7.90 \mathrm{~Hz})$, 2.67-2.62 (m, 1H), $2.36(\mathrm{~m}, 1 \mathrm{H}), 2.22-2.11(\mathrm{~m}, 3 \mathrm{H}), 2.01(\mathrm{~m}, 1 \mathrm{H}), 1.84-1.79(\mathrm{~m}, 2 \mathrm{H})$, $1.64(\mathrm{~m}, 1 \mathrm{H}), 1.54(\mathrm{~m}, 1 \mathrm{H}), 1.43-1.37(\mathrm{~m}, 1 \mathrm{H}) ;{ }^{13} \mathrm{C} \mathrm{NMR}\left(150 \mathrm{MHz}, \mathrm{CDCl}_{3}\right) \delta: 213.1,144.7,144.6$, 128.5, 127.9, 127.8, 126.2, 126.2, 48.3, 48.1, 42.2, 35.3, 34.3, 28.0, 25.0; IR (neat, $\mathrm{cm}^{-1}$ ) 1695, 1492, 1450, 1137, 747, 704, 694, 593, 531; HRMS (DART) calcd for $\mathrm{C}_{20} \mathrm{H}_{23} \mathrm{O}[\mathrm{M}+\mathrm{H}]^{+} 279.1749$, found 279.1735 .

2-(2-Phenyl-2-(p-tolyl)ethyl)cyclohexan-1-one (3ba); 90\% yield (133.3 mg); The obtained crude o mixture was roughly purified by PTLC (hexane/acetone $=9 / 1$ ), and further PTLC (hexane/ethyl acetate $=9 / 1$ ) was conducted to afford the pure product.; Colorless solid; Mp: $61-63{ }^{\circ} \mathrm{C}$; ${ }^{1} \mathrm{H}$ NMR $\left(600 \mathrm{MHz}, \mathrm{CDCl}_{3}\right.$, As a mixture of diastereomers) $\delta: 7.23(\mathrm{td}, 4 \mathrm{H}, J=14.61,6.64 \mathrm{~Hz}), 7.16-7.10(\mathrm{~m}, 3 \mathrm{H}), 7.07$ (d, 2H, $J=8.25 \mathrm{~Hz}), 4.02(\mathrm{td}, 1 \mathrm{H}, J=$ 7.90, 3.89 Hz), 2.64-2.60 (m, 1H), $2.35(\mathrm{~d}, 1 \mathrm{H}, J=13.06 \mathrm{~Hz}), 2.29(\mathrm{~d}, 3 \mathrm{H}, J=2.75 \mathrm{~Hz}), 2.18-2.15(\mathrm{~m}$, $3 \mathrm{H}), 2.00-1.99(\mathrm{~m}, 1 \mathrm{H}), 1.80-1.79(\mathrm{~m}, 2 \mathrm{H}), 1.64-1.61(\mathrm{~m}, 1 \mathrm{H}), 1.55-1.53(\mathrm{~m}, 1 \mathrm{H}), 1.40-1.38(\mathrm{~m}, 1 \mathrm{H})$;

${ }^{13} \mathrm{C}$ NMR (150 MHz, $\mathrm{CDCl}_{3}$, As a mixture of diastereomers) $\delta: 212.9,144.9,144.9,141.6,141.5$, 
135.5, 135.5, 129.1, 128.3, 127.7, 127.7, 127.6, 127.5, 126.0 (two peaks), 48.2, 48.2, 47.6 (two peaks), 42.1, 35.3, 35.3, 34.3, 34.2, 27.9, 27.9, 24.9, 20.9; IR (neat, $\mathrm{cm}^{-1}$ ) 1702, 1492, 1447, 811, 721, 698; HRMS (DART) calcd for $\mathrm{C}_{21} \mathrm{H}_{25} \mathrm{O}[\mathrm{M}+\mathrm{H}]^{+} 293.1905$, found; 293.1895 .

2-(2-Phenyl-2-(o-tolyl)ethyl)cyclohexan-1-one (3ca); 73\% yield $(107.7 \mathrm{mg})$; The product was purified by PTLC (hexane/acetone =9/1); Colorless solid; Mp: 61-63 ${ }^{\circ} \mathrm{C} ;{ }^{1} \mathrm{H}$ NMR $\left(500 \mathrm{MHz}, \mathrm{CDCl}_{3}\right.$, As a mixture of diastereomers) $\delta: 7.32-7.07$ (m, 9H), 4.32-4.26 $(\mathrm{m}, 1 \mathrm{H}), 2.61-2.55(\mathrm{~m}, 1 \mathrm{H}), 2.38-2.35(\mathrm{~m}, 1 \mathrm{H}), 2.26-2.12(\mathrm{~m}, 6 \mathrm{H}), 2.01-1.99(\mathrm{~m}, 1 \mathrm{H})$, $1.82-1.77(\mathrm{~m}, 2 \mathrm{H}), 1.68-1.53(\mathrm{~m}, 2 \mathrm{H}), 1.44-1.40(\mathrm{~m}, 1 \mathrm{H}) ;{ }^{13} \mathrm{C}$ NMR $\left(150 \mathrm{MHz}, \mathrm{CDCl}_{3}\right.$, As a mixture of diastereomers) $\delta: 213.1,213.0,144.4,144.2,142.3,142.2,136.5,136.4,130.5,128.3,128.2,128.0$, 126.5 (two peaks), 126.0, 125.9, 48.2, 48.2, 43.7, 43.6, 42.2, 35.8, 35.8, 34.6, 34.5, 28.0, 25.1, 25.0, 19.9, 19.8; IR (neat, $\mathrm{cm}^{-1}$ ) 1699, 1447, 750, 727, 700; HRMS (DART) calcd for $\mathrm{C}_{21} \mathrm{H}_{25} \mathrm{O}[\mathrm{M}+\mathrm{H}]^{+}$ 293.1905, found; 293.1913.

2-(2-(4-Methoxyphenyl)-2-phenylethyl)cyclohexan-1-one (3da); 89\% yield $(138.7 \mathrm{mg})$; The ${ }^{\mathrm{MeO}} \mathrm{O}$ obtained crude mixture was purified by PTLC (hexane/ethyl acetate $=9 / 1$ ). Colorless oil; ${ }^{1} \mathrm{H}$ NMR ( $500 \mathrm{MHz}, \mathrm{CDCl}_{3}$, As a mixture of diastereomers) $\delta: 7.26-$

$7.21(\mathrm{~m}, 4 \mathrm{H}), 7.17-7.12(\mathrm{~m}, 3 \mathrm{H}), 6.81(\mathrm{dd}, 2 \mathrm{H}, J=8.50,1.70 \mathrm{~Hz}), 4.01$ (td, $1 \mathrm{H}, J$ $=7.94,5.10 \mathrm{~Hz}), 3.77(\mathrm{~d}, 3 \mathrm{H}, J=2.83 \mathrm{~Hz}), 2.64-2.58(\mathrm{~m}, 1 \mathrm{H}), 2.37-2.35(\mathrm{~m}, 1 \mathrm{H}), 2.19-2.15(\mathrm{~m}, 3 \mathrm{H})$, 2.02-1.99 (m, 1H), 1.81-1.76 (m, 2H), 1.62-1.58 (m, 2H), 1.42-1.37 (m, 1H); ${ }^{13} \mathrm{C}$ NMR (150 MHz, $\mathrm{CDCl}_{3}$, As a mixture of diastereomers) $\delta: 213.0,157.9,145.1,145.0,136.7,136.7,128.8,128.6,128.4$, 127.7, 127.6, 126.0, 126.0, 113.8, 55.1 (two peaks), 48.3, 48.2, 47.2, 47.2, 42.1, 35.5, 35.4, 34.3, 34.2, 27.9, 27.9, 24.9; IR (neat, $\mathrm{cm}^{-1}$ ); 1705, 1609, 1510, 1448, 1247, 1177, 1034, 700; HRMS (DART) calcd for $\mathrm{C}_{21} \mathrm{H}_{25} \mathrm{O}_{2}[\mathrm{M}+\mathrm{H}]^{+}$309.1855, found; 309.1848 .

2-(2-(4-Bromophenyl)-2-phenylethyl)cyclohexan-1-one (3ea); 69\% yield (123.6 mg); The obtained<smiles>O=C1CCCC(CC(c2ccc(Br)cc2)c2ccc(Br)cc2)C1</smiles>
crude mixture was purified by PTLC (hexane/ethyl acetate $=9 / 1$ ). Colorless oil; ${ }^{1} \mathrm{H}$ NMR (500 MHz, $\mathrm{CDCl}_{3}$, As a mixture of diastereomers) $\delta: 7.40-7.37(\mathrm{~m}, 2 \mathrm{H})$, 7.29-7.26 (m, 2H), 7.21-7.16 (m, 3H), $7.10(\mathrm{dd}, 2 \mathrm{H}, J=10.20,8.50 \mathrm{~Hz}), 4.04(\mathrm{t}$, $1 \mathrm{H}, J=7.94 \mathrm{~Hz}), 2.64-2.55(\mathrm{~m}, 1 \mathrm{H}), 2.38-2.35(\mathrm{~m}, 1 \mathrm{H}), 2.23-2.09(\mathrm{~m}, 3 \mathrm{H}), 2.04-$ $2.01(\mathrm{~m}, 1 \mathrm{H}), 1.83-1.74(\mathrm{~m}, 2 \mathrm{H}), 1.62-1.57(\mathrm{~m}, 2 \mathrm{H}), 1.44-1.36(\mathrm{~m}, 1 \mathrm{H}) ;{ }^{13} \mathrm{C} \mathrm{NMR}\left(150 \mathrm{MHz}, \mathrm{CDCl}_{3}\right.$, As a mixture of diastereomers) $\delta: 212.8,143.9,143.9,143.8,143.7,131.5,131.4,129.6,129.5,128.6$, 128.5, 127.8, 127.6, 126.4, 126.3, 119.9 (two peaks), 48.2, 48.1, 47.6, 47.5, 42.2, 42.1, 35.2 (two peaks), 34.4, 34.3, 27.9, 27.9, 25.0, 24.9; IR (neat, $\mathrm{cm}^{-1}$ ) 1695, 1491, 1451, 1073, 1010, 745, 704, 695; HRMS (DART) calcd for $\mathrm{C}_{20} \mathrm{H}_{22} \mathrm{BrO}[\mathrm{M}+\mathrm{H}]^{+} 357.0854$, found; 357.0862 .

2-(2,2-Bis(4-methoxyphenyl)ethyl)cyclohexan-1-one (3fa); 87\% yield (150.2 mg); The obtained<smiles>COc1ccc(C(CC2CCCCC2=O)c2ccc(OC)cc2)cc1</smiles>
crude mixture was roughly purified by PTLC (hexane/ethyl acetate $=4 / 1$ ), and further PTLC (hexane/acetone $=7 / 1$ ) was conducted to afford the pure product. Colorless oil; ${ }^{1} \mathrm{H}$ NMR $\left(500 \mathrm{MHz}, \mathrm{CDCl}_{3}\right) \delta: 7.12$ (t, $\left.4 \mathrm{H}, J=8.50 \mathrm{~Hz}\right), 6.80$ (dd, $4 \mathrm{H}, J=8.50,1.70 \mathrm{~Hz}), 3.96(\mathrm{t}, 1 \mathrm{H}, J=7.94 \mathrm{~Hz}), 3.76(\mathrm{~d}, 6 \mathrm{H}, J=2.27 \mathrm{~Hz})$, 2.60-2.54 (m, 1H), 2.36 (dt, $1 \mathrm{H}, J=13.23,3.12 \mathrm{~Hz}), 2.23-2.11(\mathrm{~m}, 3 \mathrm{H}), 2.01-$ $1.99(\mathrm{~m}, 1 \mathrm{H}), 1.83-1.79(\mathrm{~m}, 1 \mathrm{H}), 1.77-1.71(\mathrm{~m}, 1 \mathrm{H}), 1.66-1.53(\mathrm{~m}, 2 \mathrm{H}), 1.39$ 
(ddd, $1 \mathrm{H}, J=24.09,11.90,3.40 \mathrm{~Hz}) ;{ }^{13} \mathrm{C} \mathrm{NMR}\left(125 \mathrm{MHz}, \mathrm{CDCl}_{3}\right) \delta: 213.1,157.8,137.2,137.1,128.7$, 128.6, 128.6, 128.5, 113.8, 113.7, 55.2, 55.1, 48.3, 46.4, 46.3, 42.1, 35.6, 34.2, 27.9, 25.0; IR (neat, $\mathrm{cm}^{-1}$ ) 1705, 1508, 1242, 1175, 1033, 820; HRMS (DART) calcd for $\mathrm{C}_{22} \mathrm{H}_{27} \mathrm{O}_{3}[\mathrm{M}+\mathrm{H}]^{+} 339.1960$, found; 339.1947.

2-(2,2-Bis(4-fluorophenyl)ethyl)cyclohexan-1-one (3ga); 91\% yield (143.0 mg); The obtained crude<smiles>O=C1CCCCC1CC(c1ccc(F)cc1)c1ccc(F)cc1</smiles>
mixture was purified by PTLC (hexane/ethyl acetate $=9 / 1$ ). pale yellow oil; ${ }^{1} \mathrm{H}$ $\operatorname{NMR}\left(500 \mathrm{MHz}, \mathrm{CDCl}_{3}\right) \delta: 7.18-7.12(\mathrm{~m}, 4 \mathrm{H}), 6.96(\mathrm{td}, 4 \mathrm{H}, J=8.50,3.40 \mathrm{~Hz})$, $4.05(\mathrm{t}, 1 \mathrm{H}, J=8.22 \mathrm{~Hz}), 2.60-2.54(\mathrm{~m}, 1 \mathrm{H}), 2.38-2.36(\mathrm{~m}, 1 \mathrm{H}), 2.20(\mathrm{td}, 1 \mathrm{H}, J=$ $12.90,5.86 \mathrm{~Hz}), 2.10-2.04(\mathrm{~m}, 3 \mathrm{H}), 1.84-1.81(\mathrm{~m}, 1 \mathrm{H}), 1.77-1.72(\mathrm{~m}, 1 \mathrm{H}), 1.66-$ $1.53(\mathrm{~m}, 2 \mathrm{H}), 1.44-1.39(\mathrm{~m}, 1 \mathrm{H}) ;{ }^{13} \mathrm{C} \mathrm{NMR}\left(150 \mathrm{MHz}, \mathrm{CDCl}_{3}\right) \delta: 212.8,161.3(\mathrm{~d}$, $\left.J_{\mathrm{C}-\mathrm{F}}=244.45 \mathrm{~Hz}\right), 161.3\left(\mathrm{~d}, J_{\mathrm{C}-\mathrm{F}}=244.45 \mathrm{~Hz}\right), 140.2\left(\mathrm{~d}, J_{\mathrm{C}-\mathrm{F}}=3.71 \mathrm{~Hz}\right), 140.1$ $\left(\mathrm{d}, J_{\mathrm{C}-\mathrm{F}}=3.70 \mathrm{~Hz}\right), 129.1\left(\mathrm{~d}, J_{\mathrm{C}-\mathrm{F}}=7.41 \mathrm{~Hz}\right), 129.0\left(\mathrm{~d}, J_{\mathrm{C}-\mathrm{F}}=7.41 \mathrm{~Hz}\right), 115.3\left(\mathrm{~d}, J_{\mathrm{C}-\mathrm{F}}=22.23 \mathrm{~Hz}\right)$, $115.2\left(\mathrm{~d}, J_{\mathrm{C}-\mathrm{F}}=20.37 \mathrm{~Hz}\right), 48.1,46.5,42.1,35.7,34.4,27.9,25.0 ;{ }^{19} \mathrm{~F}$ NMR $\left(565 \mathrm{MHz}, \mathrm{CDCl}_{3}\right) \delta$ : $-117.82,-117.91$; IR (neat, $\mathrm{cm}^{-1}$ ) 1702, 1510, 1222, 811, 721, 698; HRMS (DART) calcd for $\mathrm{C}_{20} \mathrm{H}_{21} \mathrm{~F}_{2} \mathrm{O}[\mathrm{M}+\mathrm{H}]^{+}$315.1555, found ; 315.1561 .

2-(2-Phenylethyl)cyclohexan-1-one (3ha); 75\% yield (133.3 mg); The product was purified by PTLC O (hexane/acetone $=9 / 1)$; The structure was confirmed by comparison with data of ${ }^{1} \mathrm{H}$ and ${ }^{13} \mathrm{C}$ NMR shown in a literature. ${ }^{17}{ }^{1} \mathrm{H} \mathrm{NMR}\left(600 \mathrm{MHz}, \mathrm{CDCl}_{3}\right) \delta$ : 7.28-7.27 (m, 2H), 7.19-7.18 (m, 3H), $2.63(\mathrm{t}, 2 \mathrm{H}, J=6.53 \mathrm{~Hz}), 2.41-2.39(\mathrm{~m}, 1 \mathrm{H}), 2.30-2.29(\mathrm{~m}, 2 \mathrm{H})$, 2.16-2.11 (m, 2H), 2.04-2.03 (m, 1H), 1.87-1.85 (m, 1H), 1.72-1.59 (m, 2H), 1.53-1.40 (m, 2H); ${ }^{13} \mathrm{C}$ NMR (150 MHz, $\left.\mathrm{CDCl}_{3}\right) \delta: 213.2,142.2,128.4,128.3,125.8,49.9,42.1,34.1,33.2,31.2,28.1,24.9$; MS (DART) $\mathrm{m} / \mathrm{z}=203$.

2-(2-(4-Tolyl)ethyl)cyclohexan-1-one (3ia); 76\% yield $(87.9 \mathrm{mg})$; The product was purified by PTLC o (hexane/acetone $=9 / 1$ ); The structure was confirmed by comparison with data of ${ }^{1} \mathrm{H}$ and ${ }^{13} \mathrm{C}$ NMR shown in a literature. ${ }^{18}{ }^{1} \mathrm{H} \mathrm{NMR}\left(600 \mathrm{MHz}, \mathrm{CDCl}_{3}\right) \delta:$ 7.07-7.07 $(\mathrm{m}, 4 \mathrm{H}), 2.59(\mathrm{t}, 2 \mathrm{H}, J=7.90 \mathrm{~Hz}), 2.40-2.38(\mathrm{~m}, 1 \mathrm{H}), 2.30-2.27(\mathrm{~m}, 5 \mathrm{H}), 2.13-$ $2.10(\mathrm{~m}, 2 \mathrm{H}), 2.03-2.01(\mathrm{~m}, 1 \mathrm{H}), 1.86-1.84(\mathrm{~m}, 1 \mathrm{H}), 1.71-1.61(\mathrm{~m}, 2 \mathrm{H}), 1.47-1.43(\mathrm{~m}, 2 \mathrm{H}) ;{ }^{13} \mathrm{C}$ NMR $\left(150 \mathrm{MHz}, \mathrm{CDCl}_{3}\right) \delta: 213.2,139.1,135.2,129.0,128.3,49.8,42.1,34.0,32.7,31.3,28.1,24.9,21.0$; MS (DART) $\mathrm{m} / \mathrm{z}=217$.

2-(2-(4-tert-Butylphenyl)ethyl)cyclohexan-1-one (3ja); 81\% yield (110.2 $\mathrm{mg})$; The product was<smiles>CC(C)(C)c1ccc(CCC2CCCCC2=O)cc1</smiles>
purified by PTLC (hexane/acetone $=9 / 1)$; Colorless oil; ${ }^{1} \mathrm{H}$ NMR $(600 \mathrm{MHz}$, $\left.\mathrm{CDCl}_{3}\right) \delta: 7.30(\mathrm{~d}, 2 \mathrm{H}, J=8.25 \mathrm{~Hz}), 7.12(\mathrm{~d}, 2 \mathrm{H}, J=8.25 \mathrm{~Hz}), 2.59(\mathrm{t}, 2 \mathrm{H}, J=$ $6.53 \mathrm{~Hz}), 2.40-2.39(\mathrm{~m}, 1 \mathrm{H}), 2.32-2.27(\mathrm{~m}, 2 \mathrm{H}), 2.16-2.10(\mathrm{~m}, 2 \mathrm{H}), 2.04-2.03$ $(\mathrm{m}, 1 \mathrm{H}), 1.86-1.86(\mathrm{~m}, 1 \mathrm{H}), 1.73-1.62(\mathrm{~m}, 2 \mathrm{H}), 1.52-1.41(\mathrm{~m}, 2 \mathrm{H}), 1.31(\mathrm{~s}, 9 \mathrm{H})$;

${ }^{13} \mathrm{C} \mathrm{NMR}\left(150 \mathrm{MHz}, \mathrm{CDCl}_{3}\right) \delta:$ 213.3, 148.5, 139.1, 128.0, 125.2, 49.9, 42.1, 34.3, 34.0, 32.6, 31.4, 31.2, 28.0, 24.9; IR (neat, $\mathrm{cm}^{-1}$ ) 1709, 1515, 1448, 1364, 1128, 834, 560; HRMS (DART) calcd for $\mathrm{C}_{18} \mathrm{H}_{27} \mathrm{O}[\mathrm{M}+\mathrm{H}]^{+}$259.2062, found 259.2063. 
2-(2-([1,1'-Biphenyl]-4-yl)ethyl)cyclohexan-1-one (3ka); 62\% yield (87.2 mg); The product was Ph) purified by PTLC (hexane/acetone $=9 / 1$ ); Colorless solid; Mp: 71-73 ${ }^{\circ} \mathrm{C} ;{ }^{1} \mathrm{H}$ NMR $\left(600 \mathrm{MHz}, \mathrm{CDCl}_{3}\right) \delta: 7.58(\mathrm{~d}, 2 \mathrm{H}, J=6.87 \mathrm{~Hz}), 7.51(\mathrm{~d}, 2 \mathrm{H}, J=8.25$ $\mathrm{Hz}), 7.42(\mathrm{t}, 2 \mathrm{H}, J=7.56 \mathrm{~Hz}), 7.32(\mathrm{t}, 1 \mathrm{H}, J=7.22 \mathrm{~Hz}), 7.26-7.25(\mathrm{~m}, 2 \mathrm{H}), 2.71-$ $2.63(\mathrm{~m}, 2 \mathrm{H}), 2.42-2.39(\mathrm{~m}, 1 \mathrm{H}), 2.33-2.29(\mathrm{~m}, 2 \mathrm{H}), 2.19-2.16(\mathrm{~m}, 2 \mathrm{H}), 2.06-$ $2.05(\mathrm{~m}, 1 \mathrm{H}), 1.88-1.87(\mathrm{~m}, 1 \mathrm{H}), 1.74-1.63(\mathrm{~m}, 2 \mathrm{H}), 1.55-1.53(\mathrm{~m}, 1 \mathrm{H}), 1.47-1.43(\mathrm{~m}, 1 \mathrm{H}) ;{ }^{13} \mathrm{C} \mathrm{NMR}$ $\left(150 \mathrm{MHz}, \mathrm{CDCl}_{3}\right) \delta: 213.2,141.1,138.8,128.8,128.7,127.1,127.0$ (two peaks), 49.8 (two peaks), 42.2, 34.0 (two peaks), 32.9, 31.2, 28.1, 25.0; IR (neat, $\mathrm{cm}^{-1}$ ) 1698, 1488, 1447, 1128, 821, 758, 721, 690, 598, 573, 546, 516, 488; HRMS (DART) calcd for $\mathrm{C}_{20} \mathrm{H}_{23} \mathrm{O}[\mathrm{M}+\mathrm{H}]^{+}$279.1749, found 279.1757.

2-(2-(4-Methoxyphenyl)ethyl)cyclohexan-1-one (3la); 90\% yield (104.4 $\mathrm{mg})$; The product was

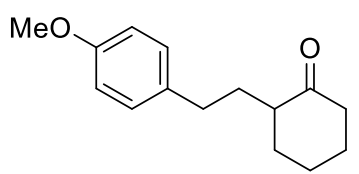
purified by PTLC (hexane/acetone $=9 / 1)$; Colorless oil; ${ }^{1} \mathrm{H}$ NMR $(600 \mathrm{MHz}$, $\left.\mathrm{CDCl}_{3}\right) \delta: 7.09(\mathrm{~d}, 2 \mathrm{H}, J=8.25 \mathrm{~Hz}), 6.82(\mathrm{~d}, 2 \mathrm{H}, J=8.25 \mathrm{~Hz}), 3.78(\mathrm{~s}, 3 \mathrm{H})$, 2.58-2.55 (m, 2H), 2.40-2.38 (m, $1 \mathrm{H}), 2.31-2.25(\mathrm{~m}, 2 \mathrm{H}), 2.14-2.07(\mathrm{~m}, 2 \mathrm{H})$, 2.05-2.02 (m, 1H), 1.86-1.84 (m, 1H), 1.70-1.61 (m, 2H), 1.49-1.39 (m, 2H);


28.0, 24.9; IR (neat, $\mathrm{cm}^{-1}$ ); 1705, 1511, 1448, 1242, 1177, 1034, 815, 518; HRMS (DART) calcd for $\mathrm{C}_{15} \mathrm{H}_{21} \mathrm{O}_{2}[\mathrm{M}+\mathrm{H}]^{+}$233.1542, found 233.1540.

2-(2-(3-Methoxyphenyl)ethyl)cyclohexan-1-one (3ma); 64\% yield $(79.1 \mathrm{mg})$; The product was o purified by PTLC (hexane/acetone =9/1); Colorless oil; ${ }^{1} \mathrm{H} \mathrm{NMR} \mathrm{(600} \mathrm{MHz,}$ $\left.\mathrm{CDCl}_{3}\right) \delta: 7.17(\mathrm{t}, 1 \mathrm{H}, J=7.90 \mathrm{~Hz}), 7.13(\mathrm{~d}, 1 \mathrm{H}, J=7.56 \mathrm{~Hz}), 6.88(\mathrm{t}, 1 \mathrm{H}, J=$ $7.56 \mathrm{~Hz}), 6.83(\mathrm{~d}, 1 \mathrm{H}, J=8.25 \mathrm{~Hz}), 3.81(\mathrm{~s}, 3 \mathrm{H}), 2.67-2.57(\mathrm{~m}, 2 \mathrm{H}), 2.41-2.39$ $(\mathrm{m}, 1 \mathrm{H}), 2.30-2.28(\mathrm{~m}, 2 \mathrm{H}), 2.20-2.15(\mathrm{~m}, 1 \mathrm{H}), 2.12-2.06(\mathrm{~m}, 1 \mathrm{H}), 2.03-2.02(\mathrm{~m}, 1 \mathrm{H}), 1.86-1.84(\mathrm{~m}$, $1 \mathrm{H}), 1.74-1.61(\mathrm{~m}, 2 \mathrm{H}), 1.50-1.40(\mathrm{~m}, 2 \mathrm{H}) ;{ }^{13} \mathrm{C} \mathrm{NMR}\left(150 \mathrm{MHz}, \mathrm{CDCl}_{3}\right) \delta: 213.5,157.4,130.6,129.8$, 127.0, 120.4, 110.2, 55.2, 50.2, 42.0, 34.0, 29.6, 28.2, 27.5, 24.8; IR (neat, $\mathrm{cm}^{-1}$ ) 1708, 1584, 1488, 1451, 1260, 1152, 1048, 783, 697; HRMS (DART) calcd for $\mathrm{C}_{15} \mathrm{H}_{21} \mathrm{O}_{2}[\mathrm{M}+\mathrm{H}]^{+}$233.1542, found 233.1546.

2-(2-(2-Methoxyphenyl)ethyl)cyclohexan-1-one (3na); 68\% yield (90.4 mg); The product was o purified by PTLC (hexane/acetone = 9/1); Colorless oil; ${ }^{1} \mathrm{H} \mathrm{NMR}\left(600 \mathrm{MHz}, \mathrm{CDCl}_{3}\right)$ $\delta: 7.19(\mathrm{t}, 1 \mathrm{H}, J=7.90 \mathrm{~Hz}), 6.77(\mathrm{~d}, 1 \mathrm{H}, J=7.56 \mathrm{~Hz}), 6.73(\mathrm{~d}, 2 \mathrm{H}, J=6.19 \mathrm{~Hz}), 3.79$ $(\mathrm{s}, 3 \mathrm{H}), 2.65-2.57(\mathrm{~m}, 2 \mathrm{H}), 2.40-2.39(\mathrm{~m}, 1 \mathrm{H}), 2.31-2.26(\mathrm{~m}, 2 \mathrm{H}), 2.15-2.12(\mathrm{~m}, 2 \mathrm{H})$, 2.04-2.02 (m, 1H), 1.86-1.85 (m, 1H), 1.72-1.61 (m, 2H), 1.52-1.39 (m, 2H); ${ }^{13} \mathrm{C}$ NMR (150 MHz, $\left.\mathrm{CDCl}_{3}\right) \delta: 213.1,159.6,143.8,129.2,120.8,114.1,111.1,55.1,49.8,42.1,34.0,33.2,31.0,28.0,24.9$; IR (neat, $\mathrm{cm}^{-1}$ ) 1708, 1599, 1494, 1242, 1120, 1030, 754; HRMS (DART) calcd for $\mathrm{C}_{15} \mathrm{H}_{21} \mathrm{O}_{2}[\mathrm{M}+$ $\mathrm{H}]^{+} 233.1542$, found 233.1535 .

2-(2-(4-Chlorophenyl)ethyl)cyclohexan-1-one (3oa); 78\% yield (97.8 mg); The product was purified<smiles>O=C1CCCCC1CCc1ccc(Cl)cc1</smiles>
by PTLC (hexane/acetone $=9 / 1)$; Colorless oil; ${ }^{1} \mathrm{H}$ NMR $\left(600 \mathrm{MHz}, \mathrm{CDCl}_{3}\right) \delta$ : $7.23(\mathrm{~d}, 2 \mathrm{H}, J=8.25 \mathrm{~Hz}), 7.11(\mathrm{~d}, 2 \mathrm{H}, J=8.25 \mathrm{~Hz}), 2.61-2.58(\mathrm{~m}, 2 \mathrm{H}), 2.41-2.38$ $(\mathrm{m}, 1 \mathrm{H}), 2.29-2.25(\mathrm{~m}, 2 \mathrm{H}), 2.15-2.03(\mathrm{~m}, 3 \mathrm{H}), 1.86-1.86(\mathrm{~m}, 1 \mathrm{H}), 1.69-1.63(\mathrm{~m}$, 2H), 1.49-1.38 (m, 2H); ${ }^{13} \mathrm{C}$ NMR (150 MHz, $\left.\mathrm{CDCl}_{3}\right) \delta: 212.9,140.6,131.4$, 
129.7, 128.4, 49.7, 42.1, 34.1, 32.6, 31.1, 28.0, 25.0; IR (neat, $\mathrm{cm}^{-1}$ ) 1708, 1492, 1448, 1130, 1090, $1015,835,805$; HRMS (DART) calcd for $\mathrm{C}_{14} \mathrm{H}_{18} \mathrm{ClO}[\mathrm{M}+\mathrm{H}]^{+}$237.1046, found 237.1044.

2-(2-(4-Acetylphenyl)ethyl)cyclohexan-1-one (3pa); 56\% yield (72.9 mg); The product was purified

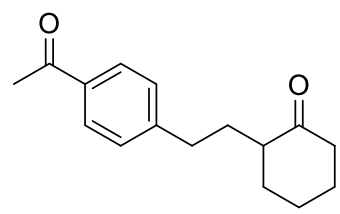
by PTLC (hexane/acetone = 9/1); Colorless solid; Mp: $71-73{ }^{\circ} \mathrm{C} ;{ }^{1} \mathrm{H}$ NMR $(600$ $\left.\mathrm{MHz}, \mathrm{CDCl}_{3}\right) \delta: 7.88(\mathrm{~d}, 2 \mathrm{H}, J=8.25 \mathrm{~Hz}), 7.28-7.27(\mathrm{~m}, 2 \mathrm{H}), 2.74-2.64(\mathrm{~m}, 2 \mathrm{H})$, $2.58(\mathrm{~s}, 3 \mathrm{H}), 2.42-2.39(\mathrm{~m}, 1 \mathrm{H}), 2.31-2.27(\mathrm{~m}, 2 \mathrm{H}), 2.17-2.12(\mathrm{~m}, 2 \mathrm{H}), 2.07-2.06$ $(\mathrm{m}, 1 \mathrm{H}), 1.88-1.87(\mathrm{~m}, 1 \mathrm{H}), 1.69-1.64(\mathrm{~m}, 2 \mathrm{H}), 1.53-1.50(\mathrm{~m}, 1 \mathrm{H}), 1.45-1.42(\mathrm{~m}$, $1 \mathrm{H}) ;{ }^{13} \mathrm{C} \mathrm{NMR}\left(150 \mathrm{MHz}, \mathrm{CDCl}_{3}\right.$ ) $\delta: 212.8,197.8,148.1,135.1,128.6$ (two peaks), 128.5, 126.7, 49.8, 42.2, 34.1, 33.3, 30.9, 28.0, 26.5, 25.0; IR (neat, $\mathrm{cm}^{-1}$ ) 1698, 1672, 1604, 1411, 1355, 1267, 1128, 950, 851, 843, 824, 717, 690, 603, 588, 573; HRMS (DART) calcd for $\mathrm{C}_{16} \mathrm{H}_{21} \mathrm{O}_{2}[\mathrm{M}+\mathrm{H}]^{+} 245.1542$, found 245.1535 .

2-(2-(4-Cyanophenyl)ethyl)cyclohexan-1-one (3qa); 65\% yield (71.5 mg); The product was purified

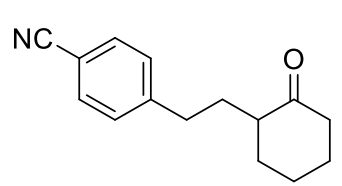
by PTLC (hexane/acetone $=9 / 1)$; Colorless oil; ${ }^{1} \mathrm{H}$ NMR $\left(600 \mathrm{MHz}, \mathrm{CDCl}_{3}\right) \delta$ : $7.56(\mathrm{~d}, 2 \mathrm{H}, J=7.56 \mathrm{~Hz}), 7.29-7.28(\mathrm{~m}, 2 \mathrm{H}), 2.74-2.63(\mathrm{~m}, 2 \mathrm{H}), 2.41-2.40(\mathrm{~m}$, $1 \mathrm{H}), 2.31-2.28(\mathrm{~m}, 2 \mathrm{H}), 2.14-2.07(\mathrm{~m}, 3 \mathrm{H}), 1.88-1.87(\mathrm{~m}, 1 \mathrm{H}), 1.69-1.64(\mathrm{~m}$, $2 \mathrm{H}), 1.50-1.43(\mathrm{~m}, 2 \mathrm{H}) ;{ }^{13} \mathrm{C}$ NMR $\left(150 \mathrm{MHz}, \mathrm{CDCl}_{3}\right) \delta: 212.6,148.0,132.2$, 129.1, 119.1, 109.7, 49.8, 42.2, 34.2, 33.5, 30.9, 28.0, 25.0; IR (neat, $\mathrm{cm}^{-1}$ ) 1704, 1608, 1504, 1448, 1128, 844, 818, 554; HRMS (DART) calcd for $\mathrm{C}_{15} \mathrm{H}_{18} \mathrm{NO}[\mathrm{M}+\mathrm{H}]^{+} 228.1388$, found 228.1398.

2-(2-(2-Naphthyl)ethyl)cyclohexan-1-one (3ra); $42 \%$ yield $(53.5 \mathrm{mg})$; The product was purified by PTLC (hexane/acetone $=9 / 1)$; Colorless solid; Mp: $61-63{ }^{\circ} \mathrm{C} ;{ }^{1} \mathrm{H}$ NMR $(600$ $\left.\mathrm{MHz}, \mathrm{CDCl}_{3}\right)$ 8: 7.80-7.75 (m, 3H), 7.61 (s, 1H), 7.46-7.40 (m, 2H), 7.34 (d, 1H, $J=7.56 \mathrm{~Hz}), 2.84-2.76(\mathrm{~m}, 2 \mathrm{H}), 2.41-2.40(\mathrm{~m}, 1 \mathrm{H}), 2.34-2.16(\mathrm{~m}, 4 \mathrm{H}), 2.05-$ $2.03(\mathrm{~m}, 1 \mathrm{H}), 1.87-1.85(\mathrm{~m}, 1 \mathrm{H}), 1.74-1.56(\mathrm{~m}, 3 \mathrm{H}), 1.46-1.44(\mathrm{~m}, 1 \mathrm{H}) ;{ }^{13} \mathrm{C} \mathrm{NMR}\left(150 \mathrm{MHz}, \mathrm{CDCl}_{3}\right)$ 8: 213.2, 139.7, 133.6, 132.0, 127.9, 127.6, 127.4, 127.3, 126.3, 125.9, 125.1, 49.8, 42.2, 34.1, 33.3, 31.0, 28.1, 25.0; IR (neat, $\mathrm{cm}^{-1}$ ) 1704, 1505, 1445, 1202, 1125, 910, 861, 815, 748, 476; HRMS (DART) calcd for $\mathrm{C}_{18} \mathrm{H}_{21} \mathrm{O}[\mathrm{M}+\mathrm{H}]^{+}$253.1592, found 253.1585.

2-(2,3-Dihydro-1H-inden-2-yl)cyclohexan-1-one (3sa); $82 \%$ yield $(89.8 \mathrm{mg})$; The product was

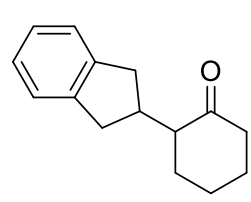
purified by PTLC (hexane/acetone $=9 / 1$ ); The structure was confirmed by comparison with data of $1 \mathrm{H}$ and ${ }^{13} \mathrm{C}$ NMR shown in a literature. ${ }^{19}{ }^{1} \mathrm{H}$ NMR $(600 \mathrm{MHz}$, $\left.\mathrm{CDCl}_{3}\right) \delta:$ 7.18-7.17 (m, 2H), 7.12-7.11 (m, 2H), $3.23(\mathrm{dd}, 1 \mathrm{H}, J=8.02,4.01 \mathrm{~Hz})$, $3.03(\mathrm{dd}, 1 \mathrm{H}, J=7.79,3.89 \mathrm{~Hz}), 2.76(\mathrm{td}, 1 \mathrm{H}, J=17.18,8.94 \mathrm{~Hz}), 2.64(\mathrm{dd}, 1 \mathrm{H}, J=$ 15.46, 9.28 Hz), 2.54 (dd, $1 \mathrm{H}, J=15.81,8.94 \mathrm{~Hz}), 2.46-2.40(\mathrm{~m}, 2 \mathrm{H}), 2.35-2.33(\mathrm{~m}, 1 \mathrm{H}), 2.16-2.14$ $(\mathrm{m}, 1 \mathrm{H}), 2.05-2.03(\mathrm{~m}, 1 \mathrm{H}), 1.90-1.89(\mathrm{~m}, 1 \mathrm{H}), 1.77-1.65(\mathrm{~m}, 2 \mathrm{H}), 1.55-1.50(\mathrm{~m}, 1 \mathrm{H}) ;{ }^{13} \mathrm{C}$ NMR $(150$ $\left.\mathrm{MHz}, \mathrm{CDCl}_{3}\right) \delta: 213.2,143.6,142.7,126.2,126.0,124.3,124.2,56.3,42.3,39.4,38.2,36.7,32.2$, 28.3, 24.7; MS (DART) $\mathrm{m} / \mathrm{z}=215$. 
2-Cyclopentylmethylcyclohexan-1-one (3ta); 78\% yield $(71.3 \mathrm{mg})$; The product was purified by O PTLC (hexane/acetone $=9 / 1)$; Colorless liquid; ${ }^{1} \mathrm{H}$ NMR $\left(600 \mathrm{MHz}, \mathrm{CDCl}_{3}\right) \delta: 2.37-$ $2.31(\mathrm{~m}, 3 \mathrm{H}), 2.13-2.11(\mathrm{~m}, 1 \mathrm{H}), 2.02-2.00(\mathrm{~m}, 1 \mathrm{H}), 1.85-1.36(\mathrm{~m}, 12 \mathrm{H}), 1.21-1.20(\mathrm{~m}$, 1H), 1.06-1.03 (m, 2H); $\left.{ }^{13} \mathrm{C} \mathrm{NMR} \mathrm{(150} \mathrm{MHz,} \mathrm{CDCl}_{3}\right) \delta: 213.8,49.8,41.9,37.4,35.6$, 34.1 (two peaks), 33.0, 32.4, 28.1, 25.0, 24.7; IR (neat, $\mathrm{cm}^{-1}$ ) 1708, 1448, 1127, 734, 521; HRMS (DART) calcd for $\mathrm{C}_{12} \mathrm{H}_{21} \mathrm{O}[\mathrm{M}+\mathrm{H}]^{+}$181.1592, found 181.1591 .

2-Cyclohexylmethylcyclohexan-1-one (3ua); 56\% yield $(54.4 \mathrm{mg})$; The product was purified by O PTLC (hexane/acetone $=9 / 1$ ); The structure was confirmed by comparison with data of ${ }^{1} \mathrm{H}$ and ${ }^{13} \mathrm{C}$ NMR shown in a literature. ${ }^{20}{ }^{1} \mathrm{H}$ NMR (600 MHz, $\left.\mathrm{CDCl}_{3}\right) \delta: 2.40-2.27$ $(\mathrm{m}, 3 \mathrm{H}), 2.08-2.01(\mathrm{~m}, 2 \mathrm{H}), 1.85-1.84(\mathrm{~m}, 1 \mathrm{H}), 1.73-1.58(\mathrm{~m}, 8 \mathrm{H}), 1.39-1.33(\mathrm{~m}, 1 \mathrm{H})$, $1.25-1.10(\mathrm{~m}, 4 \mathrm{H}), 1.06-1.01(\mathrm{~m}, 1 \mathrm{H}), 0.87-0.83(\mathrm{~m}, 2 \mathrm{H}) ;{ }^{13} \mathrm{C} \mathrm{NMR}\left(150 \mathrm{MHz}, \mathrm{CDCl}_{3}\right) \delta: 213.9,47.8$, 42.0, 36.9, 34.8, 34.2, 33.8, 33.0, 28.1, 26.6, 26.3 (two peaks), 24.8; MS (DART) m/z = 195.

2-(2,2-Diphenylethyl)cyclopentan-1-one (3ab); 92\% yield (126.2 mg); The obtained crude mixture $O$ was purified by PTLC (hexane/ethyl acetate $=9 / 1$ ). Colorless solid; Mp: $111-114{ }^{\circ} \mathrm{C}$;

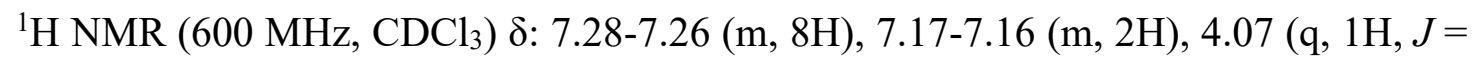
$5.27 \mathrm{~Hz}), 2.68-2.63(\mathrm{~m}, 1 \mathrm{H}), 2.27(\mathrm{q}, 1 \mathrm{H}, J=8.94 \mathrm{~Hz}), 2.19-2.18(\mathrm{~m}, 1 \mathrm{H}), 2.13-2.07$ (m, 1H), 1.98-1.97 (m, 1H), 1.89-1.86 (m, 2H), 1.68-1.65 (m, 1H), 1.53-1.50 (m, 1H); ${ }^{3} \mathrm{C}$ NMR (150 $\left.\mathrm{MHz}_{2} \mathrm{CDCl}_{3}\right) \delta: 221.0,144.8,143.5,128.5,128.4,127.8,127.6,126.3,126.1,48.9,47.2,37.9,35.8$, 29.9, 20.5; IR (neat, $\mathrm{cm}^{-1}$ ) 1725, 1492, 1451, 1157, 705, 697; HRMS (DART) calcd for $\mathrm{C}_{19} \mathrm{H}_{21} \mathrm{O}$ [M $+\mathrm{H}]^{+}$265.1592, found; 265.1584 .

2-(2,2-Diphenylethyl)cycloheptan-1-one (3ac); 96\% yield (139.5 mg); The obtained crude mixture

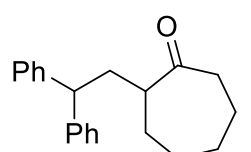
was purified by PTLC (hexane/ethyl acetate $=9 / 1$ ). Colorless solid; Mp: $54-57{ }^{\circ} \mathrm{C} ;{ }^{1} \mathrm{H}$ $\operatorname{NMR}\left(600 \mathrm{MHz}, \mathrm{CDCl}_{3}\right)$ 8: 7.27-7.23 (m, 8H), 7.17-7.16 (m, 2H), $3.97(\mathrm{t}, 1 \mathrm{H}, J=$ $7.90 \mathrm{~Hz}), 2.53-2.49(\mathrm{~m}, 1 \mathrm{H}), 2.45-2.41(\mathrm{~m}, 2 \mathrm{H}), 2.34-2.32(\mathrm{~m}, 1 \mathrm{H}), 1.99-1.95(\mathrm{~m}, 1 \mathrm{H})$, $1.84-1.78(\mathrm{~m}, 4 \mathrm{H}), 1.47-1.45(\mathrm{~m}, 1 \mathrm{H}), 1.38(\mathrm{q}, 1 \mathrm{H}, J=11.23 \mathrm{~Hz}), 1.30(\mathrm{q}, 2 \mathrm{H}, J=11.23 \mathrm{~Hz}) ;{ }^{13} \mathrm{C}$ NMR (150 MHz, $\left.\mathrm{CDCl}_{3}\right) \delta: 215.7,144.4,144.4,128.4,128.3,127.9,127.7,126.1,126.1,49.4,48.2$, 42.8, 37.7, 31.4, 29.0, 28.3, 24.1; IR (neat, $\mathrm{cm}^{-1}$ ) 1698, 1494, 1451, 1031, 744, 697; HRMS (DART) calcd for $\mathrm{C}_{21} \mathrm{H}_{25} \mathrm{O}[\mathrm{M}+\mathrm{H}]^{+}$293.1905, found; 293.1920 .

2-(2,2-Diphenylethyl)-4-methylcyclohexan-1-one (3ad); 90\% yield (133.7 $\mathrm{mg})$; The product was<smiles>CC1CCC(=O)C(Cc2ccccc2)C1</smiles>
purified by PTLC (hexane/acetone = 9/1); Colorless solid; Mp: 67-69 ${ }^{\circ} \mathrm{C}$; ${ }^{1} \mathrm{H}$ NMR $\left(600 \mathrm{MHz}, \mathrm{CDCl}_{3}\right.$, As a mixture of diastereomers) $\delta: 7.26-7.22(\mathrm{~m}$, major $8 \mathrm{H}$, minor $8 \mathrm{H}), 7.18-7.16(\mathrm{~m}$, major $2 \mathrm{H}$, minor $2 \mathrm{H}), 4.08(\mathrm{t}$, minor $1 \mathrm{H}, J=7.90 \mathrm{~Hz}), 3.96(\mathrm{t}$, major $1 \mathrm{H}, J=7.90 \mathrm{~Hz}), 2.67-2.62(\mathrm{~m}$, minor $1 \mathrm{H}), 2.57-2.53(\mathrm{~m}$, major $1 \mathrm{H}), 2.39-2.22(\mathrm{~m}$, major $3 \mathrm{H}$, minor $2 \mathrm{H}), 2.18-2.17(\mathrm{~m}$, minor $1 \mathrm{H}), 2.12-1.91(\mathrm{~m}$, major $3 \mathrm{H}$, minor $2 \mathrm{H}), 1.84-1.82(\mathrm{~m}$, minor $1 \mathrm{H}), 1.77-1.71(\mathrm{~m}$, major $2 \mathrm{H}$, minor $1 \mathrm{H}), 1.56-1.55(\mathrm{~m}$, major $1 \mathrm{H}), 1.38-1.31(\mathrm{~m}$, minor $1 \mathrm{H})$, 1.13 (q, minor $1 \mathrm{H}, J=12.37 \mathrm{~Hz}$ ), 0.98-0.97 (m, major $3 \mathrm{H}$, minor $3 \mathrm{H}) ;{ }^{13} \mathrm{C} \mathrm{NMR}\left(150 \mathrm{MHz}, \mathrm{CDCl}_{3}\right.$, As a mixture of diastereomers) $\delta: 214.1,213.0,144.7,144.6,144.3$ (two peaks), 128.4, 127.8, 127.7, 126.2, 126.1, 48.1, 47.9, 47.0, 45.1, 42.6, 41.5, 39.6, 37.8, 36.3, 35.8, 35.1, 33.9, 31.7, 26.6, 21.2, 19.5; IR (neat, $\mathrm{cm}^{-1}$ ) 1695, 1492, 1451, 1135, 1030, 744, 704, 694, 533; HRMS (DART) calcd for $\mathrm{C}_{21} \mathrm{H}_{25} \mathrm{O}$ 
$[\mathrm{M}+\mathrm{H}]^{+}$293.1905, found; 293.1915.

2-(2,2-Diphenylethyl)-3-methylcyclohexan-1-one (3ae); 58\% yield (87.6 mg); The product was O purified by PTLC (hexane/acetone $=9 / 1$ ); The desired product was obtained in high diastereoselectivity ( $>99 / 1)$, and the major related configuration was determined by Xray single crystal structure analysis (See section 2-2-5). Colorless solid; Mp: 102$104{ }^{\circ} \mathrm{C} ;{ }^{1} \mathrm{H} \mathrm{NMR} \quad\left(600 \mathrm{MHz}, \mathrm{CDCl}_{3}\right) \delta: 7.30-7.25(\mathrm{~m}, 6 \mathrm{H}), 7.20(\mathrm{~d}, 3 \mathrm{H}, J=7.56 \mathrm{~Hz}), 7.15-7.14(\mathrm{~m}$, $1 \mathrm{H}), 4.12(\mathrm{q}, 1 \mathrm{H}, J=5.27 \mathrm{~Hz}), 2.47-2.42(\mathrm{~m}, 1 \mathrm{H}), 2.36-2.34(\mathrm{~m}, 1 \mathrm{H}), 2.19(\mathrm{td}, 1 \mathrm{H}, J=12.72,5.50$ Hz), 2.12-2.08 (m, 1H), 1.98-1.95 (m, 1H), 1.89 (t, 1H, $J=9.62 \mathrm{~Hz}), 1.80$ (dd, 1H, $J=13.75,2.75$ $\mathrm{Hz}), 1.35(\mathrm{dt}, 1 \mathrm{H}, J=28.18,7.22 \mathrm{~Hz}) 1.03(\mathrm{~d}, 3 \mathrm{H}, J=6.19 \mathrm{~Hz}) ;{ }^{13} \mathrm{C} \mathrm{NMR}\left(150 \mathrm{MHz}, \mathrm{CDCl}_{3}\right) \delta: 213.0$, 145.6, 143.9, 128.5, 128.3 (two peaks), 127.6, 126.2, 125.9, 55.2, 49.1, 41.9, 40.0, 33.7, 33.0, 26.2, 20.6; IR (neat, $\mathrm{cm}^{-1}$ ) 1697, 1492, 1448, 1120, 751, 698, 551; HRMS (DART) calcd for $\mathrm{C}_{21} \mathrm{H}_{25} \mathrm{O}[\mathrm{M}+$ $\mathrm{H}]^{+}$293.1905, found; 293.1917.

4-Methyl-6,6-diphenylhexan-3-one (3af); 71\% yield (98.3 mg); The product was purified by PTLC

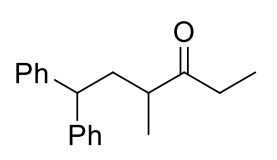
(hexane/acetone =9/1); Colorless oil; ${ }^{1} \mathrm{H}$ NMR $\left(500 \mathrm{MHz}, \mathrm{CDCl}_{3}\right) \delta$ : 7.29-7.16 (m, $10 \mathrm{H}), 3.91(\mathrm{t}, 1 \mathrm{H}, J=7.94 \mathrm{~Hz}), 2.47-2.38(\mathrm{~m}, 3 \mathrm{H}), 2.24-2.20(\mathrm{~m}, 1 \mathrm{H}), 2.01-1.95(\mathrm{~m}$, $1 \mathrm{H}), 1.10(\mathrm{~d}, 3 \mathrm{H}, J=6.80 \mathrm{~Hz}), 0.97(\mathrm{t}, 3 \mathrm{H}, J=7.37 \mathrm{~Hz}) ;{ }^{13} \mathrm{C} \mathrm{NMR}\left(150 \mathrm{MHz}, \mathrm{CDCl}_{3}\right)$ $\delta: 214.9,144.2,128.5,128.5,127.8$ (two peaks), 126.3, 126.2, 48.8, 43.7, 38.6, 34.5, 17.2, 7.7; IR (neat, $\mathrm{cm}^{-1}$ ) 1709, 1494, 1451, 1375, 1104, 1030, 974, 748, 698; HRMS (DART) calcd for $\mathrm{C}_{19} \mathrm{H}_{23} \mathrm{O}$ $[\mathrm{M}+\mathrm{H}]^{+}$267.1749, found; 267.1761 .

2-Methyl-1,4,4-triphenylbutan-1-one (3ag); 79\% yield (124.7 mg); The product was purified by<smiles>CC(CC(c1ccccc1)c1ccccc1)C(=O)c1ccccc1</smiles>
PTLC (hexane/acetone =9/1); Colorless oil; ${ }^{1} \mathrm{H} \mathrm{NMR}\left(600 \mathrm{MHz}, \mathrm{CDCl}_{3}\right) \delta: 7.67(\mathrm{~d}$, $2 \mathrm{H}, J=7.56 \mathrm{~Hz}), 7.51(\mathrm{t}, 1 \mathrm{H}, J=7.56 \mathrm{~Hz}), 7.37(\mathrm{t}, 2 \mathrm{H}, J=7.56 \mathrm{~Hz}), 7.29-7.25(\mathrm{~m}$, $6 \mathrm{H}), 7.21-7.16(\mathrm{~m}, 4 \mathrm{H}), 3.99(\mathrm{t}, 1 \mathrm{H}, J=7.90 \mathrm{~Hz}), 3.34-3.33(\mathrm{~m}, 1 \mathrm{H}), 2.63-2.58(\mathrm{~m}$, 1H), 2.14-2.09 (m, 1H), $1.22(\mathrm{~d}, 3 \mathrm{H}, J=6.87 \mathrm{~Hz}) ;{ }^{13} \mathrm{C} \mathrm{NMR}\left(150 \mathrm{MHz}, \mathrm{CDCl}_{3}\right) \delta: 204.1,144.3,144.2$, 136.4, 132.8, 128.5, 128.2, 127.9, 126.3 (two peaks), 48.7, 39.4, 38.3, 17.5; IR (neat, cm ${ }^{-1}$ ) 1679, 1494, 1448, 1230, 971, 750, 700; HRMS (DART) calcd for $\mathrm{C}_{23} \mathrm{H}_{23} \mathrm{O}[\mathrm{M}+\mathrm{H}]^{+} 315.1749$, found; 315.1755 . 


\section{2-5. Determination of related configuration of 3ae}

3ae was dissolved in chloroform $(0.4 \mathrm{~mL})$, and the whole was put at room temperature under hexane vapor atmosphere. After 3 days, small colorless crystals appeared and were collected by filtration. Then, X-ray single crystal structure analysis was conducted using the obtained crystal (Figures S3, S4). It was found that the configuration of the major diastereomer was anti. The analytical data was available on website of The Cambridge Crystallographic Data Centre (CCDC 2080743).
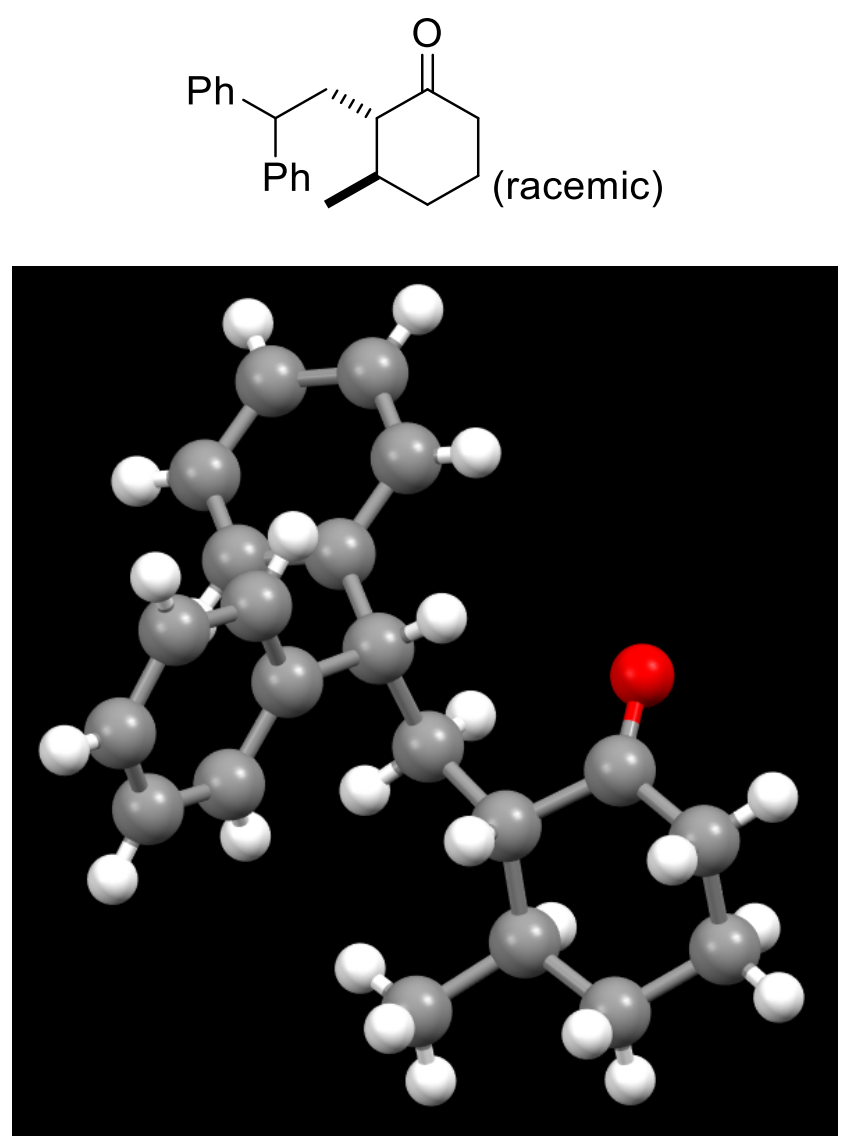

Figure S3. X-ray structure of 3ae 


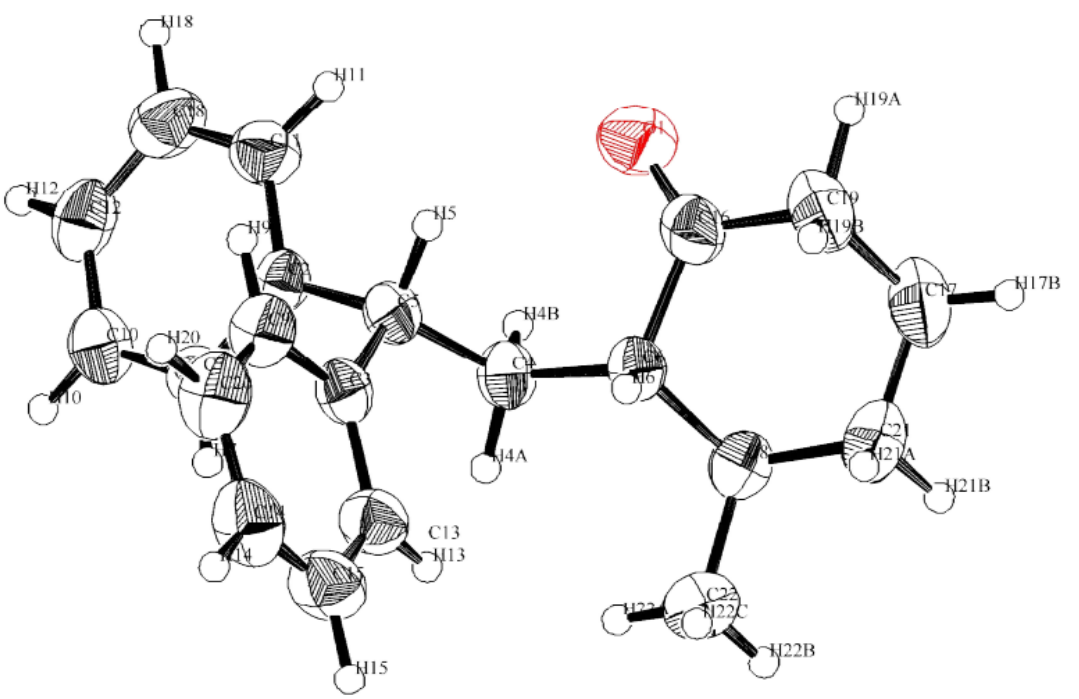

Figure S4. X-ray structure (ORTEP, probability: 50\%) of 3ae 


\section{References}

[1] Luo, J.; Zhang, J. Donor-Acceptor Fluorophores for Visible-Light-Promoted Organic Synthesis: Photoredox/Ni Dual Catalytic C(sp3)-C(sp2) Cross-Coupling, ACS Catal. 2016, 6, 873-877.

[2] Romero, N. A.; Nicewicz, D. A. Organic Photoredox Catalysis, Chem. Rev. 2016, 116, 1007510166.

[3] Wayner, D. D. M.; McPhee, D. J.; Griller, D. Oxidation and reduction potentials of transient free radicals, J. Am. Chem. Soc. 1988, 110, 132-137.

[4] Sibi, M. P.; Hasegawa, M. Organocatalysis in Radical Chemistry. Enantioselective $\alpha-$ Oxyamination of Aldehydes J. Am. Chem. Soc. 2007, 129, 4124-4125.

[5] Bas, S.; Yamashita, Y.; Kobayashi, S. Development of Brønsted Base-Photocatalyst Hybrid Systems for Highly Efficient C-C Bond Formation Reactions of Malonates with Styrenes, ACS Catal. 2020, 10, 10546-10550.

[6] Stein, T.; Peréz, M.; Dobrovetsky, R.; Winkelhaus, D.; Caputo, C. B.; Stephan, D. W. Electrophilic Fluorophosphonium Cations in Frustrated Lewis Pair Hydrogen Activation and Catalytic Hydrogenation of Olefins, Angew. Chem. Int. Ed. 2015, 54, 10178-10182.

[7] Tang, M.; Han, S.; Huang, S.; Huang, S.; Xie, L.-G. Carbosulfenylation of Alkenes with Organozinc Reagents and Dimethyl(methylthio)sulfonium Trifluoromethanesulfonate, Org. Lett. 2020, 22, 9729-9734.

[8] Okita, T.; Asahara, K. K.; Muto, K.; Yamaguchi, J. Palladium-Catalyzed Mizoroki-Heck Reaction of Nitroarenes and Styrene Derivatives, Org. Lett. 2020, 22, 3205-3208.

[9] Clark, R. D.; Untch, K. G. [2 + 2] Cycloaddition of ethyl propiolate and silyl enol ethers, J. Org. Chem. 1979, 44, 248-253.

[10]Xie, W.; Kim, D.; Chang, S. Copper-Catalyzed Formal Dehydrogenative Coupling of Carbonyls with Polyfluoroarenes Leading to $\beta-\mathrm{C}-\mathrm{H}$ Arylation, J. Am. Soc. Chem. 2020, 142, 20588-20593.

[11] Orban, J.; Turner, J. V.; Twitchin, B. Preparation of tert-butyldimethylsilyl (TBDMS) enol ethers using potassium hydride in the presence of TBDMS chloride Tetrahedron Lett. 1984, 25, 50995102.

[12]Esumi, N.; Suzuki, K.; Nishimoto, Y.; Yasuda, M. Synthesis of 1,4-Dicarbonyl Compounds from Silyl Enol Ethers and Bromocarbonyls, Catalyzed by an Organic Dye under Visible-Light Irradiation with Perfect Selectivity for the Halide Moiety over the Carbonyl Group, Org. Lett. 2016, 18, 5704-5707.

[13]Tandiary, M. A.; Asano, M.; Hattori, T.; Takehira, S.; Masui, Y.; Onaka, M. Unprecedented alkylation of silicon enolates with alcohols via carbenium ion formations catalyzed by tin hydroxide-embedded montmorillonite, Tetrahedron Lett. 2017, 58, 1925-1928.

[14]Li, J.-R.; Tao, Y.; Yu, Q.; Bu, X.-H. A pcu-type metal-organic framework with spindle $\left[\mathrm{Zn}_{7}(\mathrm{OH})_{8}\right]^{6+}$ cluster as secondary building units Chem. Commun. 2007, 1527-1529.

[15]Çakmak, O.; Kahveci, I.; Demirtaş, Í.; Hökelek, T.; Smith, K. Bromination of Tetralin. Short and Efficient Synthesis of 1,4-Dibromonaphthalene, Collect. Czech. Chem. Commun. 2000, 65, 17911804.

[16]Johnson, C. R.; Marren, T. J. Trimethylsilyl chloride/tetramethylethylenediamine facilitated additions of organocopper reagents $(\mathrm{RCu})$ to enones, Tetrahedron Lett. 1987, 28, 27-30.

[17] Soorukram, D.; Knochel, P. Copper - Catalyzed Preparation of Ketones Bearing a Stereogenic Center in $\alpha$ Position, Angew. Chem. Int. Ed. 2006, 45, 3686-3689. 
[18]Nithiy, N.; Orellana, A. Palladium-Catalyzed Cross-Coupling of Benzyl Chlorides with Cyclopropanol-Derived Ketone Homoenolates, Org. Lett. 2014, 16, 5854-5857.

[19]Bornowski, E. C.; Hinds, E. M.; White, D. R.; Nakamura, Y.; Wolfe, J. P. Pd-Catalyzed Alkene Difunctionalization Reactions of Enolates for the Synthesis of Substituted Bicyclic Cyclopentanes, Org. Process Res. Dev. 2019, 23, 1610-1630.

[20]Wei, Y.; Rao, B.; Cong, X.; Zeng, X. Highly Selective Hydrogenation of Aromatic Ketones and Phenols Enabled by Cyclic (Amino)(alkyl)carbene Rhodium Complexes, J. Am. Chem. Soc. 2015, $137,9250-9253$. 


\section{NMR charts}

${ }^{1} \mathrm{H} \mathrm{NMR}$ of DCA $\left(500 \mathrm{MHz}, \mathrm{CDCl}_{3}\right)$<smiles>N#Cc1c2ccccc2c(C#N)c2ccccc12</smiles>

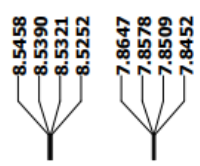

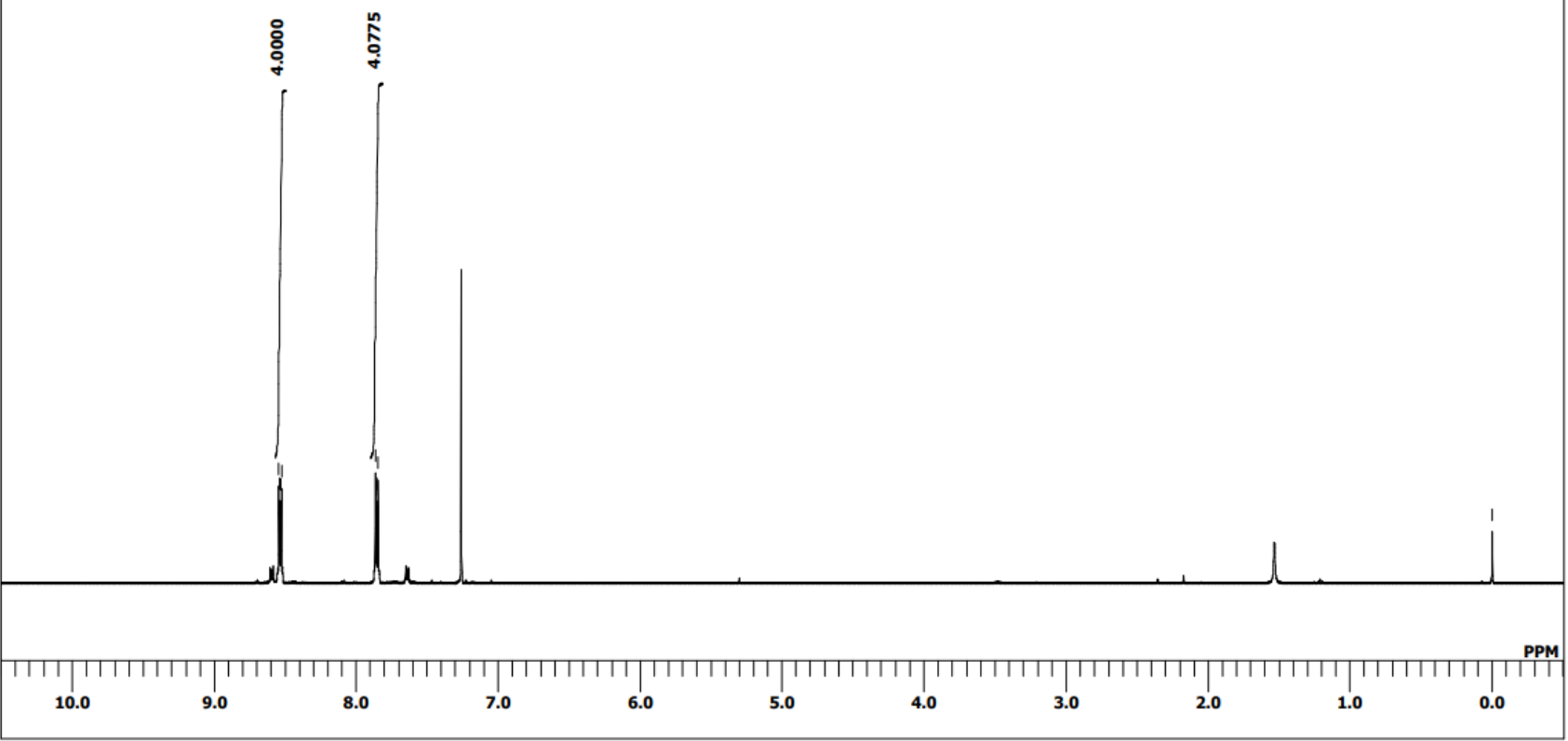


${ }^{1} \mathrm{H}$ NMR of DCN $\left(600 \mathrm{MHz}, \mathrm{CDCl}_{3}\right)$

CN




${ }^{1} \mathrm{H}$ NMR of $4 \mathrm{CzIPN}\left(600 \mathrm{MHz}, \mathrm{CDCl}_{3}\right)$

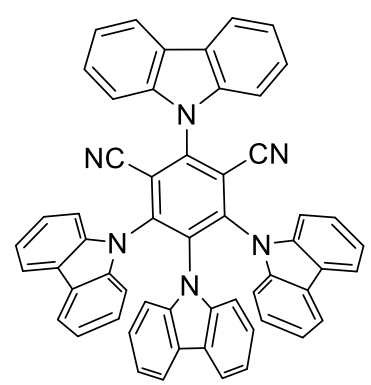



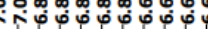

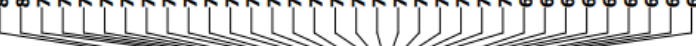

( רוा

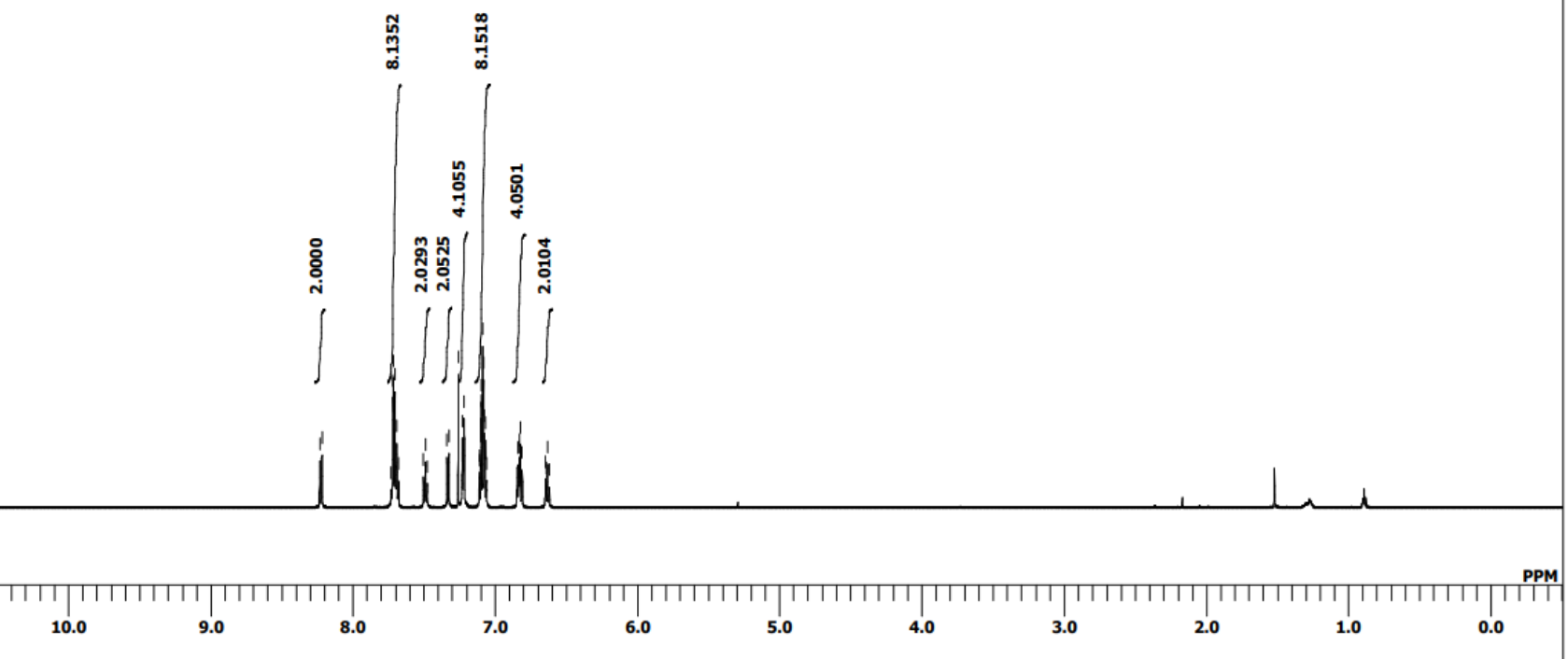


${ }^{1} \mathrm{H}$ NMR of 4DPAIPN $\left(600 \mathrm{MHz}, \mathrm{CDCl}_{3}\right)$

${ }^{1} \mathrm{H}$ NMR of 4CzTPN $\left(600 \mathrm{MHz}, \mathrm{CDCl}_{3}\right)$
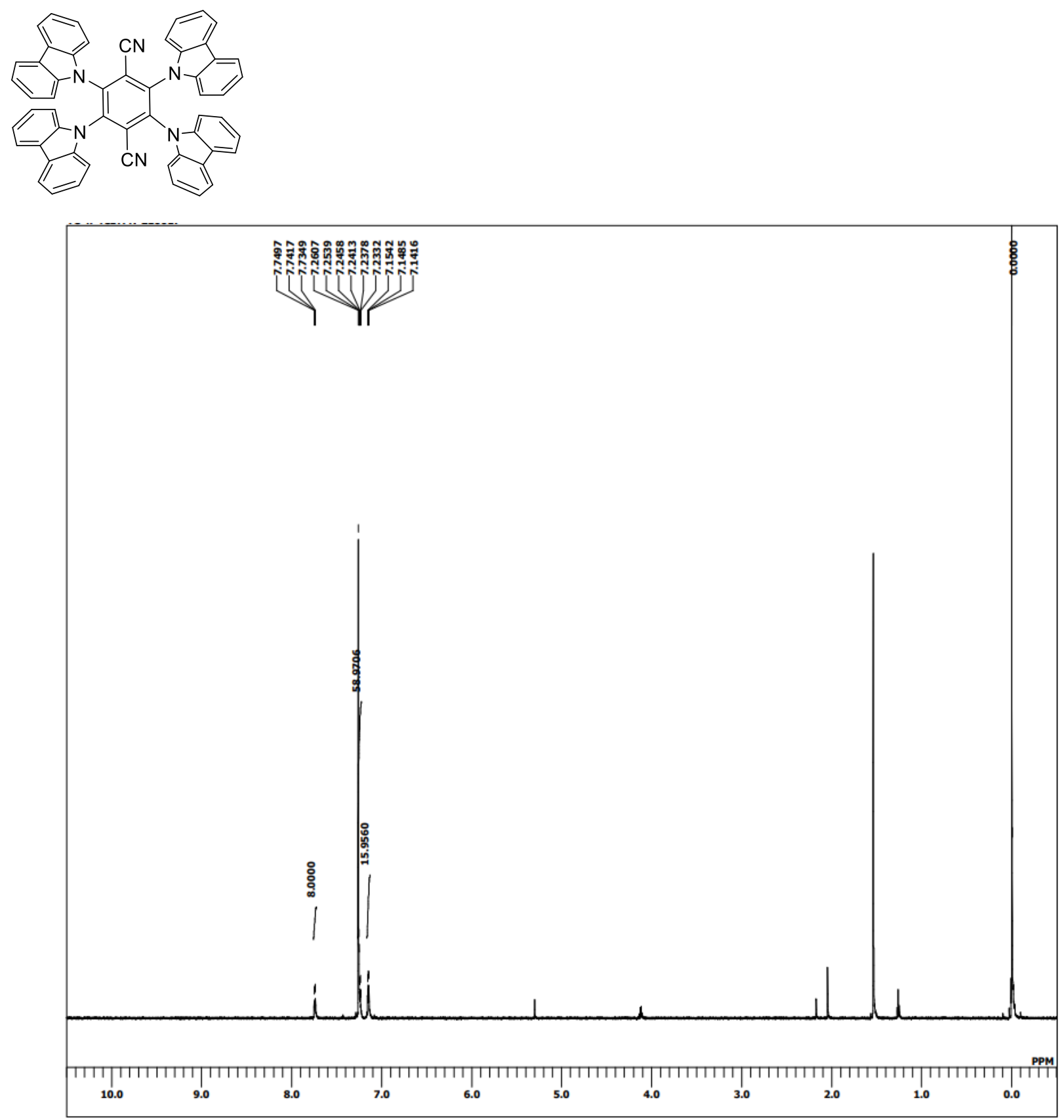
${ }^{1} \mathrm{H}$ NMR of $1 \mathbf{a}\left(500 \mathrm{MHz}, \mathrm{CDCl}_{3}\right)$

4

${ }^{1} \mathrm{H}$ NMR of $\mathbf{1 b}\left(500 \mathrm{MHz}, \mathrm{CDCl}_{3}\right)$<smiles>C=C(c1ccccc1)c1ccc(C)cc1</smiles>




${ }^{1} \mathrm{H}$ NMR of $1 \mathrm{c}\left(500 \mathrm{MHz}, \mathrm{CDCl}_{3}\right)$<smiles>C=C(c1ccccc1)c1ccccc1C</smiles>

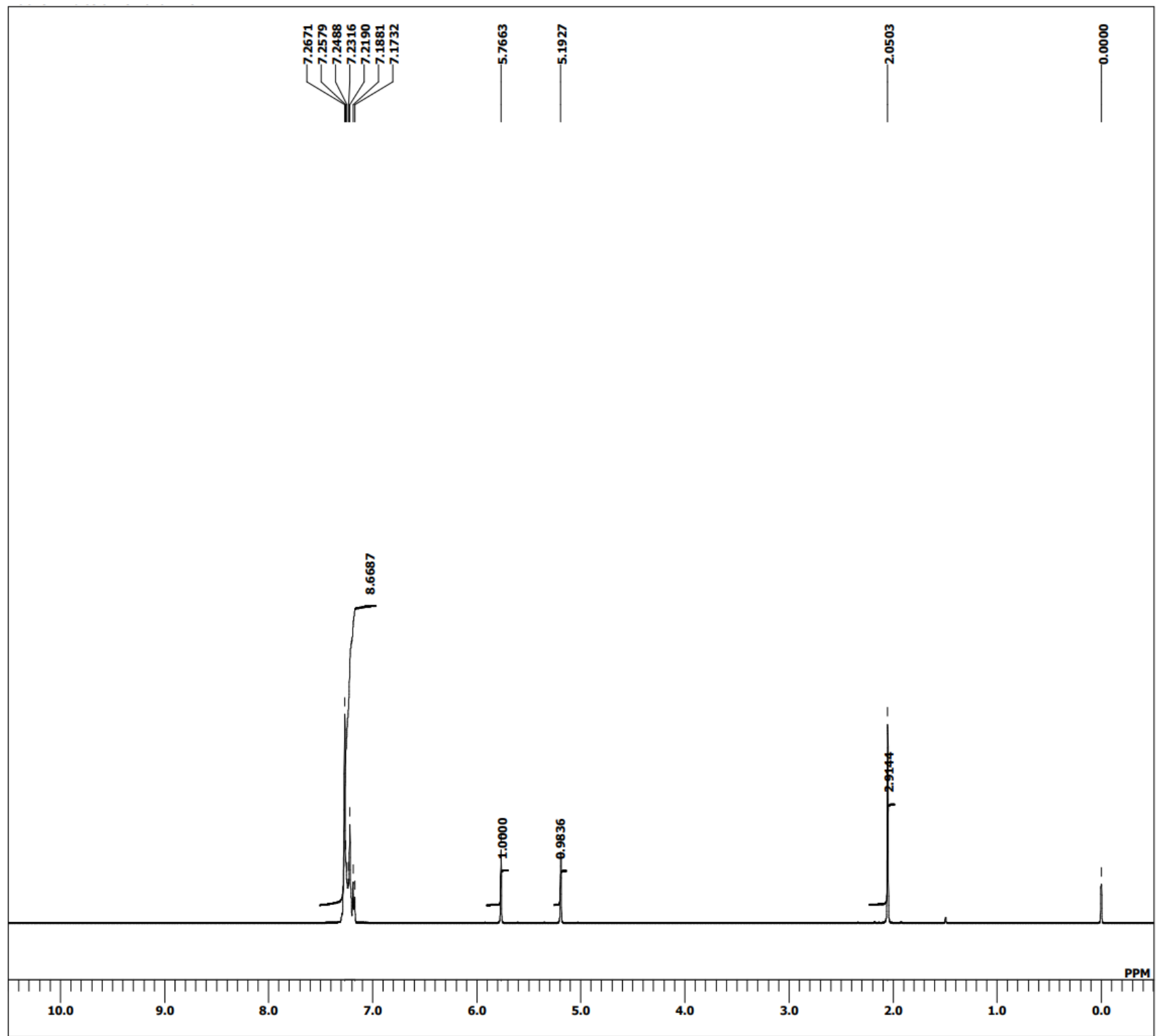


${ }^{1} \mathrm{H}$ NMR of $\mathbf{1 d}\left(500 \mathrm{MHz}, \mathrm{CDCl}_{3}\right)$

$\mathrm{MeO}$<smiles>C=C(c1ccccc1)c1ccccc1</smiles>

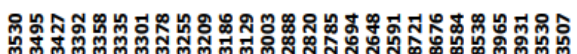

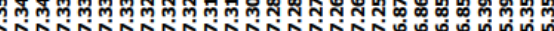




${ }^{1} \mathrm{H}$ NMR of 1 e $\left(500 \mathrm{MHz}, \mathrm{CDCl}_{3}\right)$
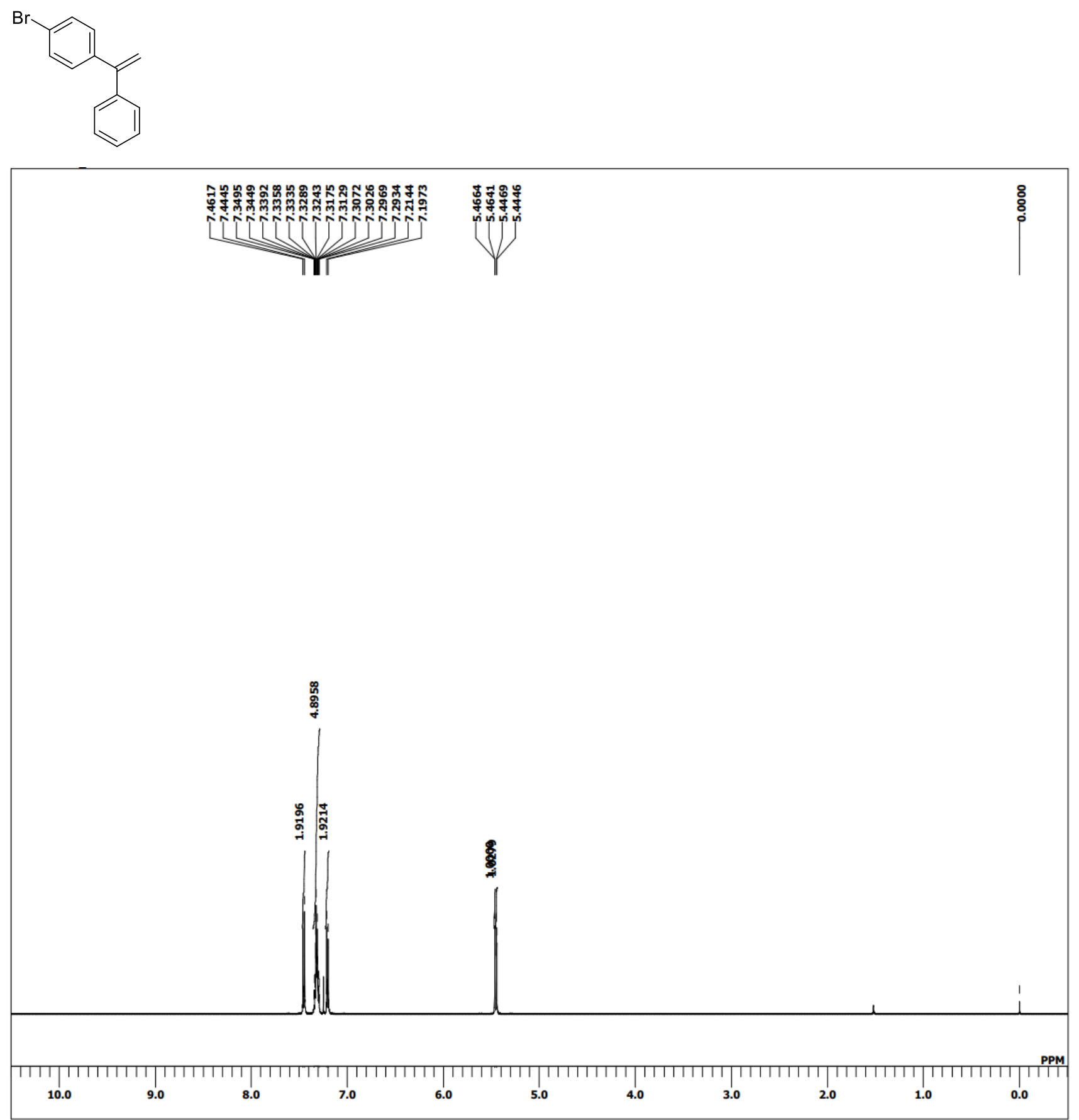
${ }^{1} \mathrm{H} \mathrm{NMR}$ of $\mathbf{1 f}\left(600 \mathrm{MHz}, \mathrm{CDCl}_{3}\right)$
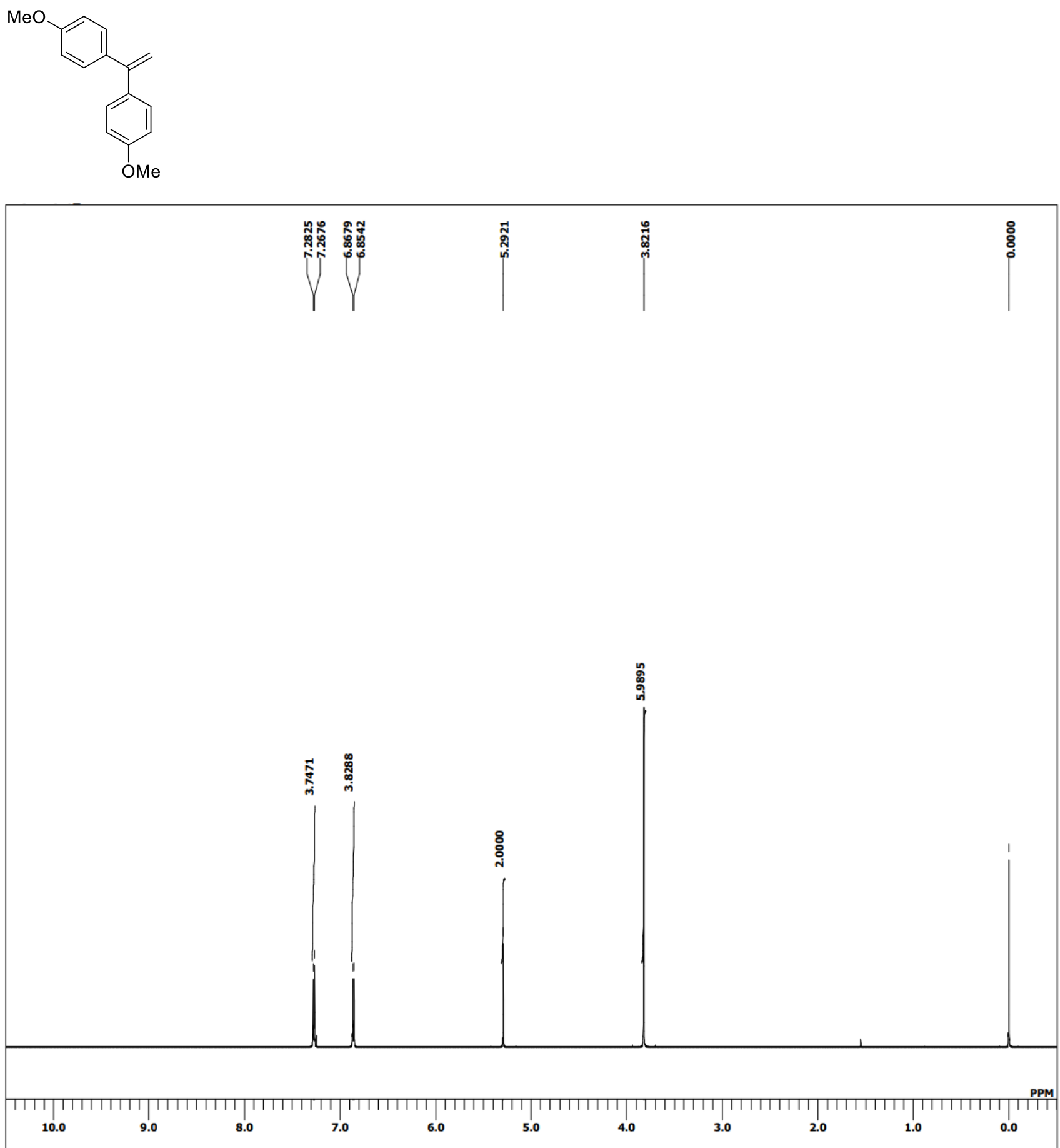
${ }^{1} \mathrm{H}$ NMR of 1 g $\left(600 \mathrm{MHz}, \mathrm{CDCl}_{3}\right)$

${ }^{1} \mathrm{H}$ NMR of $1 \mathbf{k}\left(500 \mathrm{MHz}, \mathrm{CDCl}_{3}\right)$

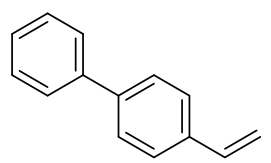

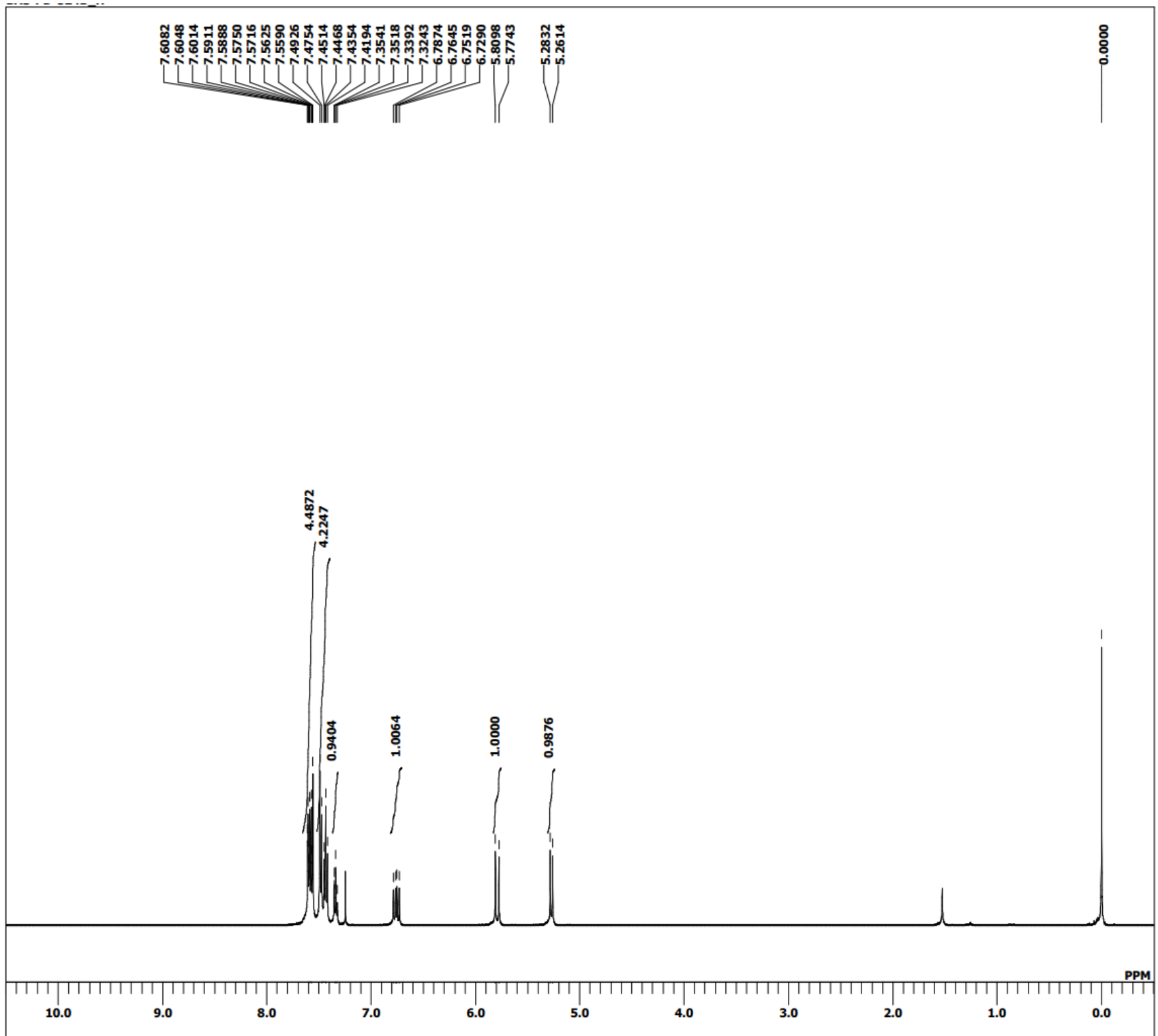


${ }^{1} \mathrm{H}$ NMR of $11\left(500 \mathrm{MHz}, \mathrm{CDCl}_{3}\right)$

$\mathrm{MeO}$<smiles>C=Cc1ccccc1</smiles>

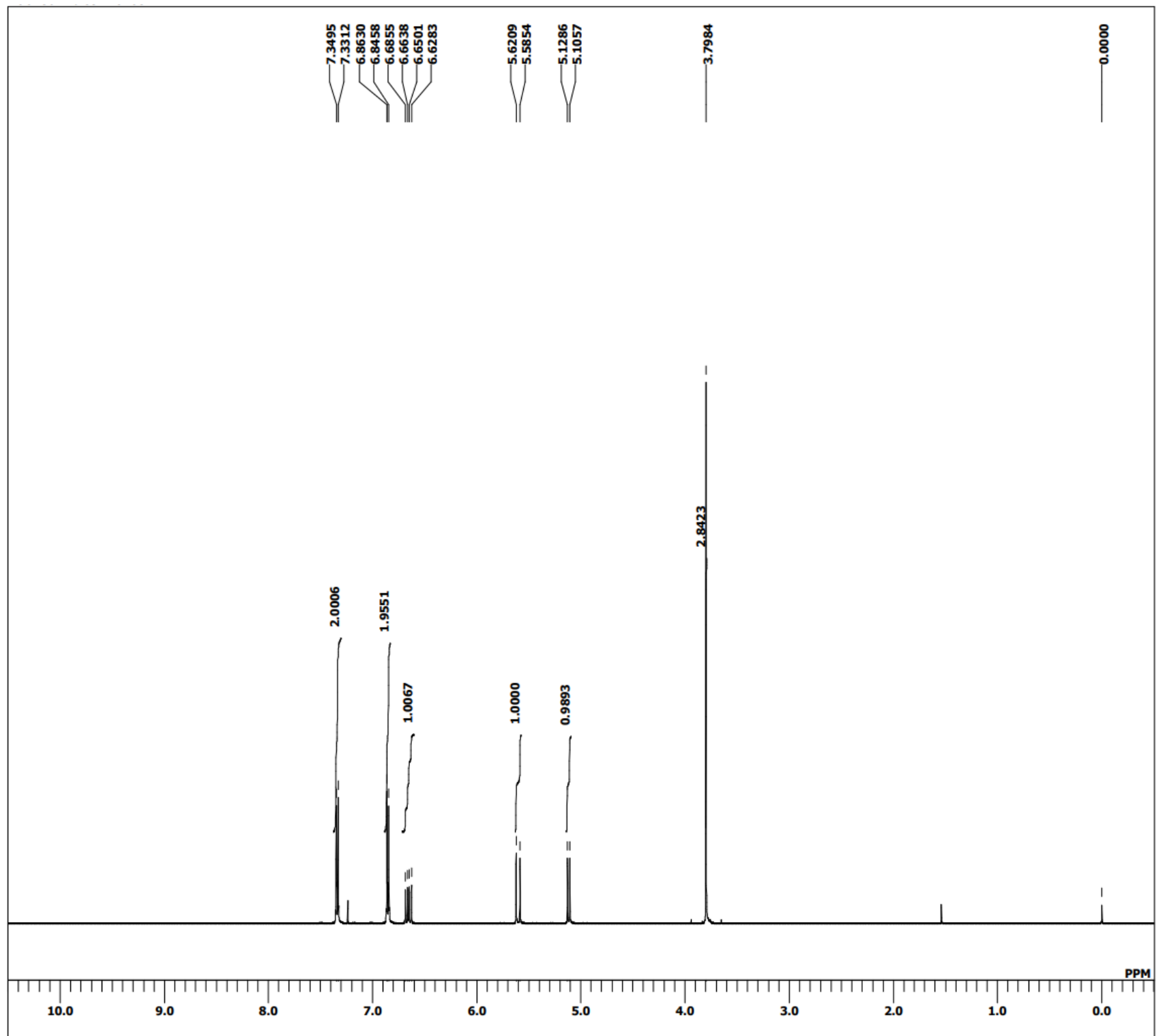


${ }^{1} \mathrm{H}$ NMR of 1 m $\left(600 \mathrm{MHz}, \mathrm{CDCl}_{3}\right)$

MeO

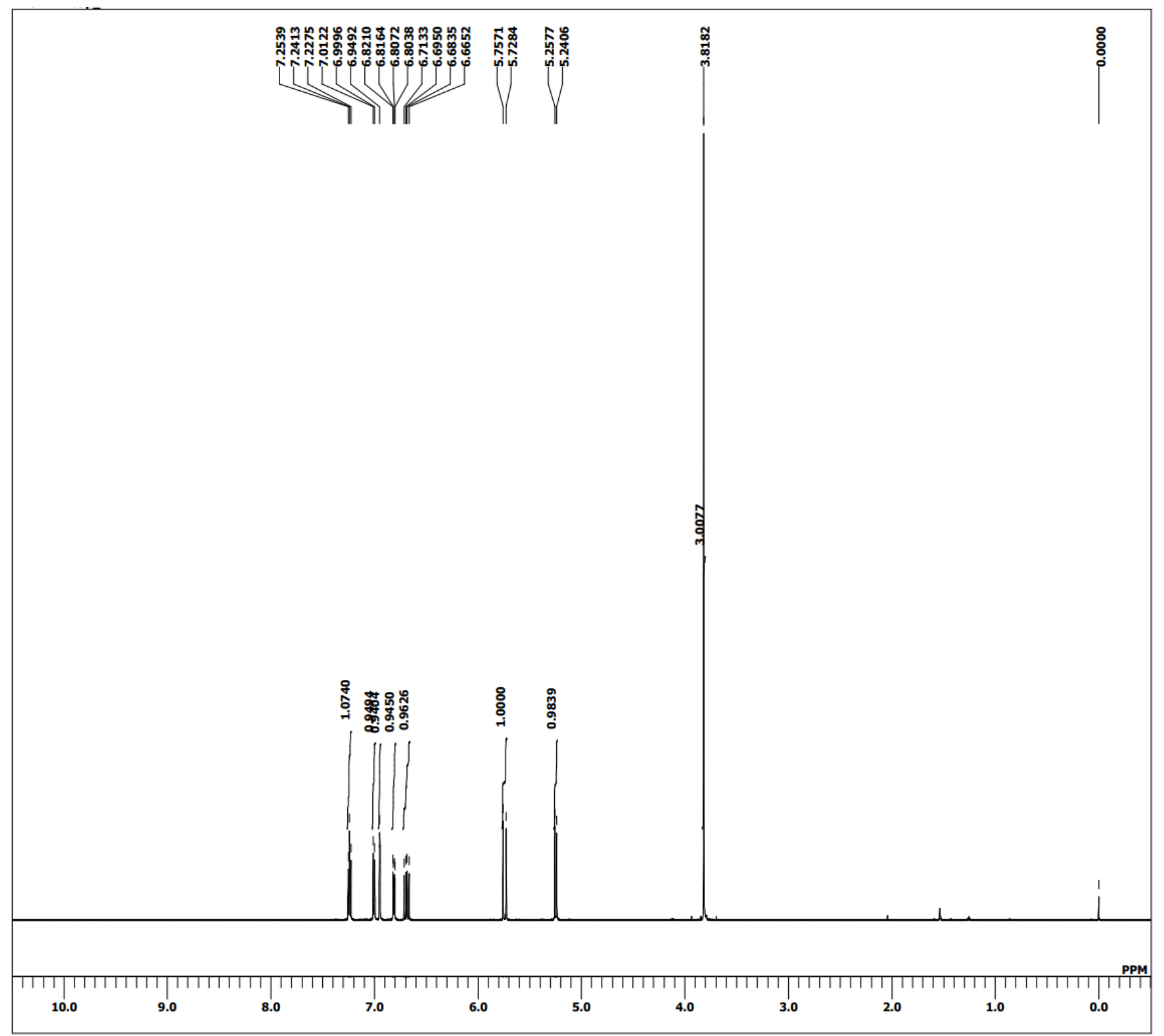


${ }^{1} \mathrm{H}$ NMR of 1 n $\left(600 \mathrm{MHz}, \mathrm{CDCl}_{3}\right)$

$\overbrace{}^{\mathrm{OMe}}$

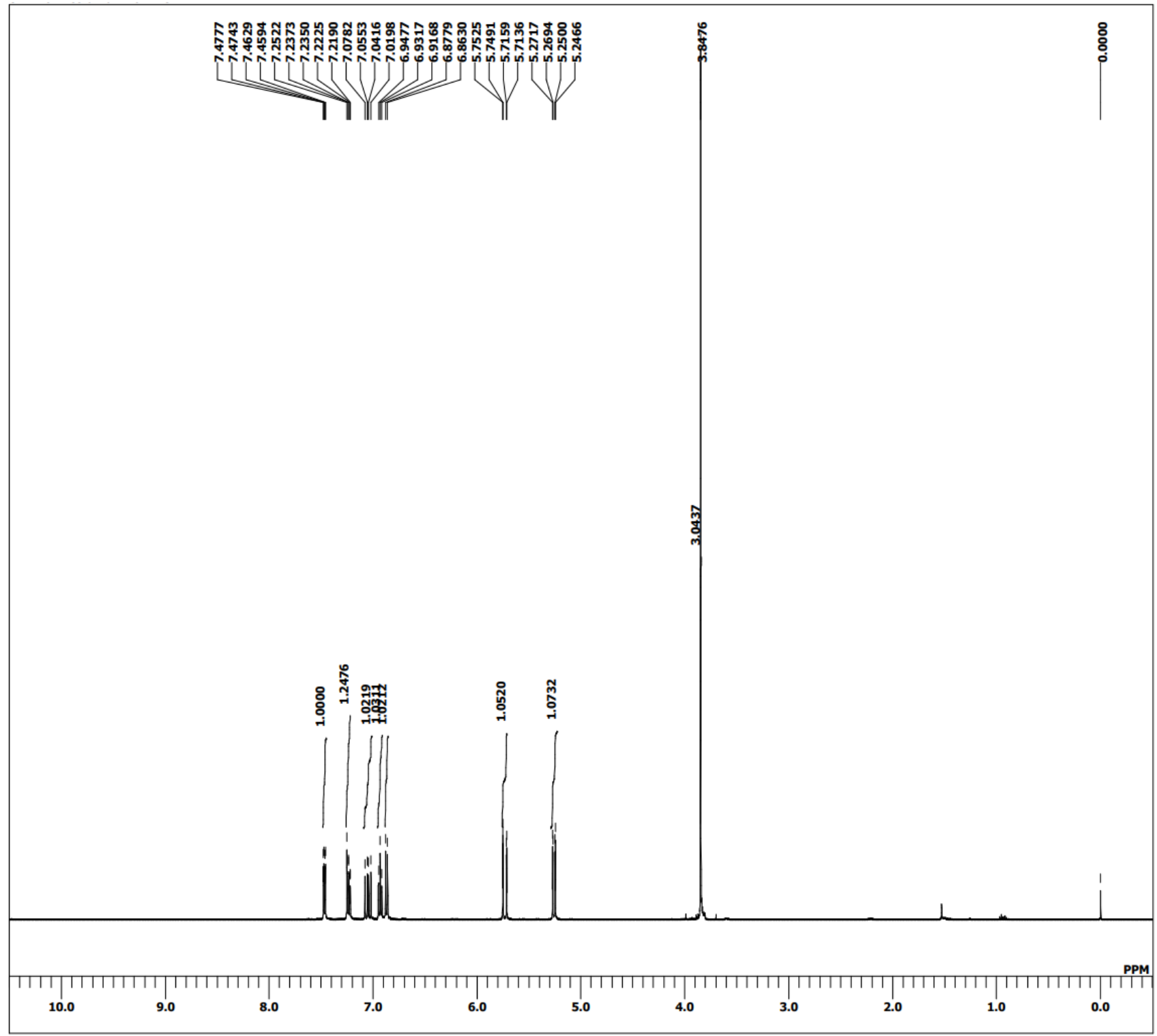


${ }^{1} \mathrm{H}$ NMR of $1 p\left(600 \mathrm{MHz}, \mathrm{CDCl}_{3}\right)$

${ }^{1} \mathrm{H}$ NMR of 1q $\left(600 \mathrm{MHz}, \mathrm{CDCl}_{3}\right)$

NC

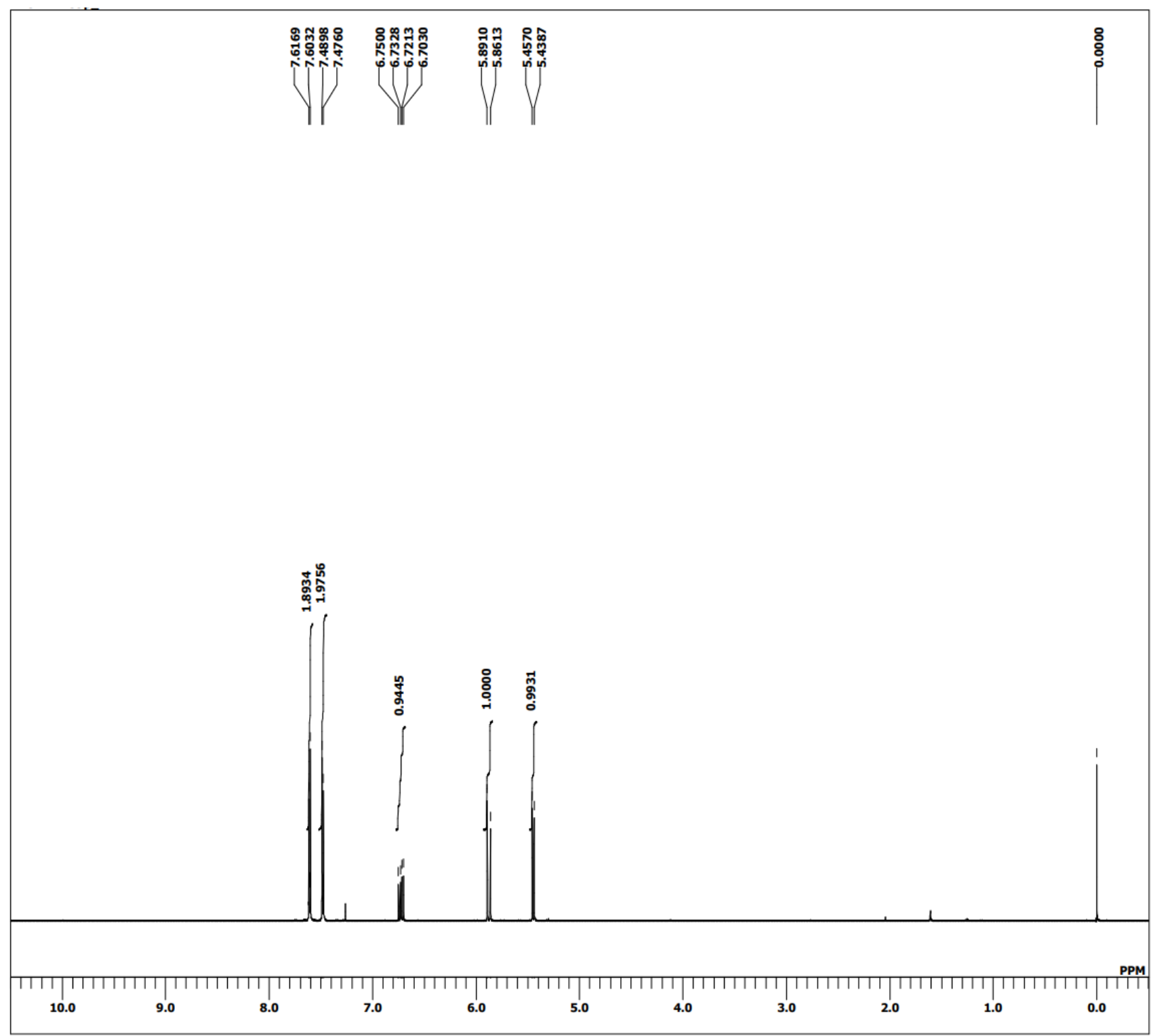


${ }^{1} \mathrm{H} \mathrm{NMR}$ of $1 \mathbf{r}\left(600 \mathrm{MHz}, \mathrm{CDCl}_{3}\right)$




${ }^{1} \mathrm{H}$ NMR of $\mathbf{2 a}\left(600 \mathrm{MHz}, \mathrm{CDCl}_{3}\right)$

OTBS

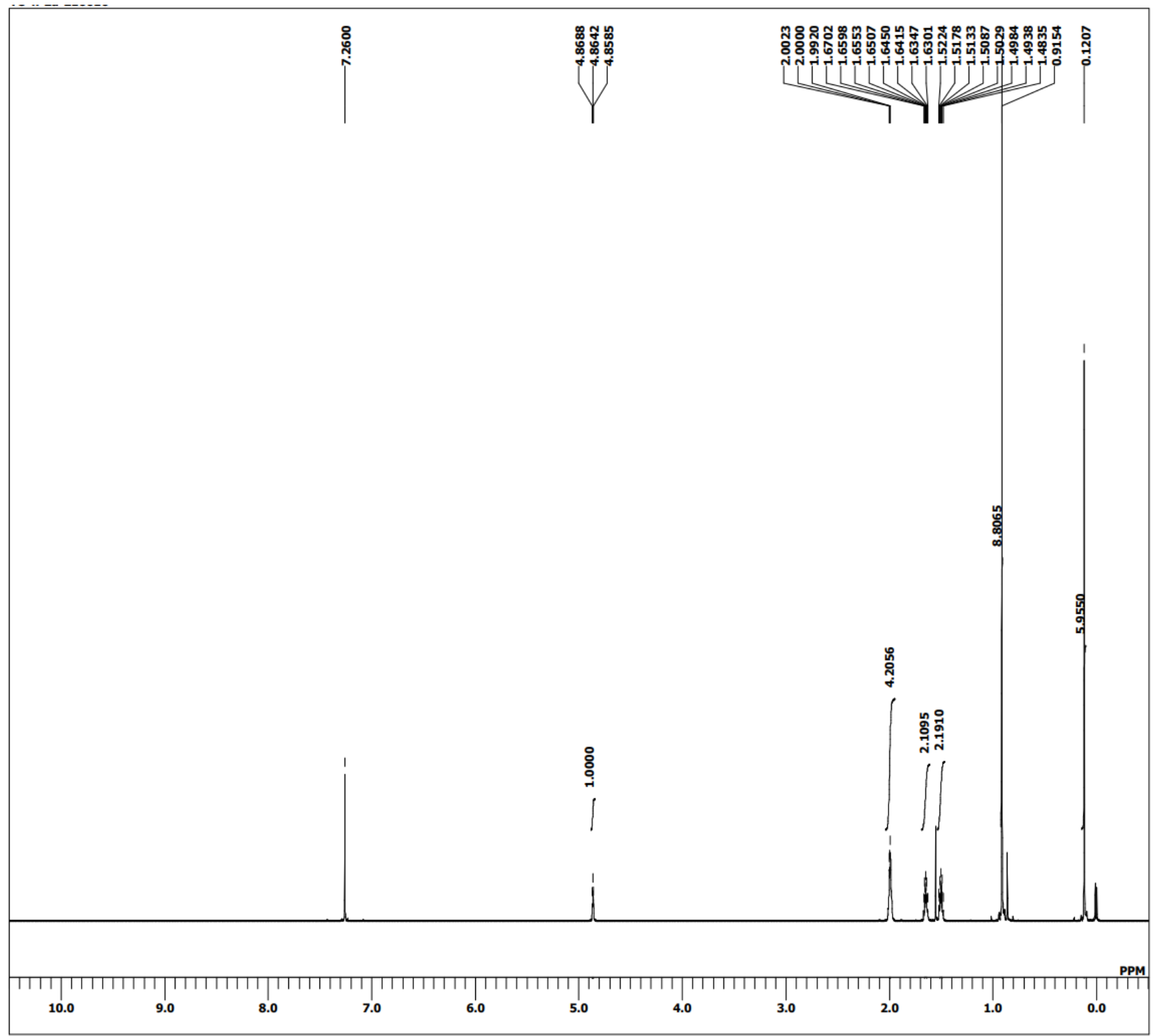


${ }^{1} \mathrm{H} \mathrm{NMR}$ of $\mathbf{2 b}\left(600 \mathrm{MHz}, \mathrm{CDCl}_{3}\right)$

OTBS

$\Longrightarrow$

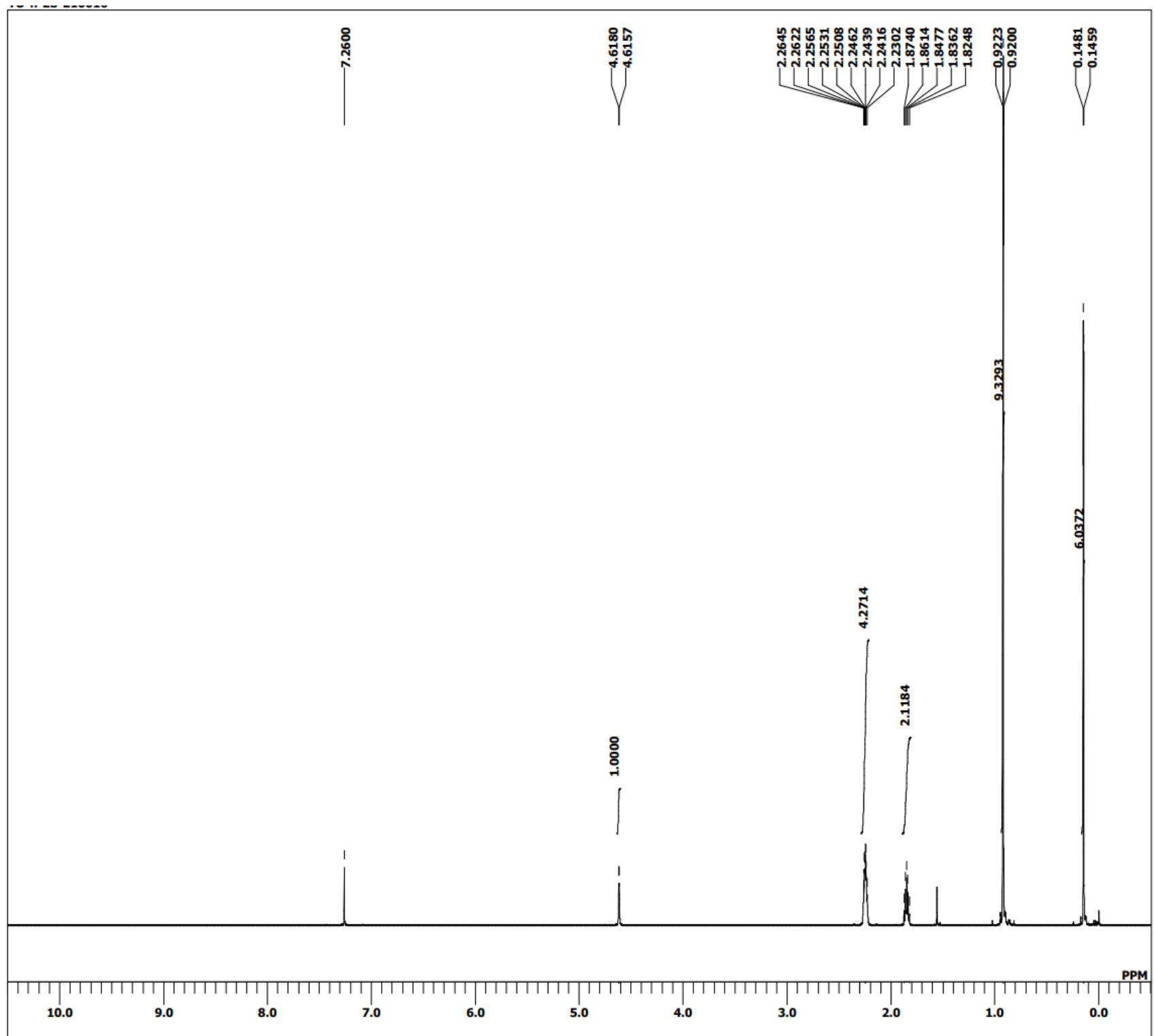


${ }^{1} \mathrm{H}$ NMR of $2 \mathbf{c}\left(600 \mathrm{MHz}, \mathrm{CDCl}_{3}\right)$
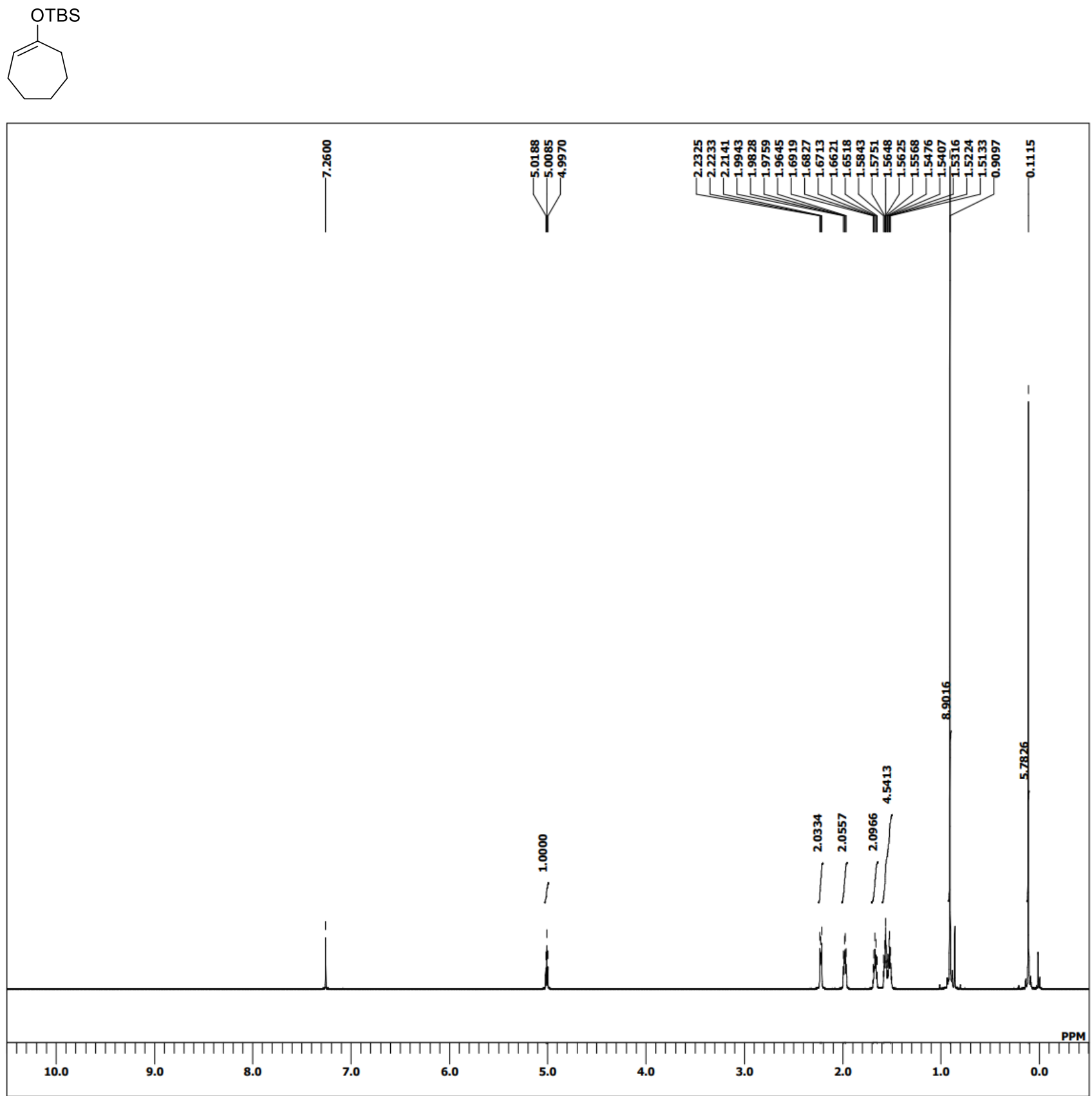
${ }^{1} \mathrm{H} \mathrm{NMR}$ of $2 \mathbf{d}\left(600 \mathrm{MHz}, \mathrm{CDCl}_{3}\right)$

OTBS

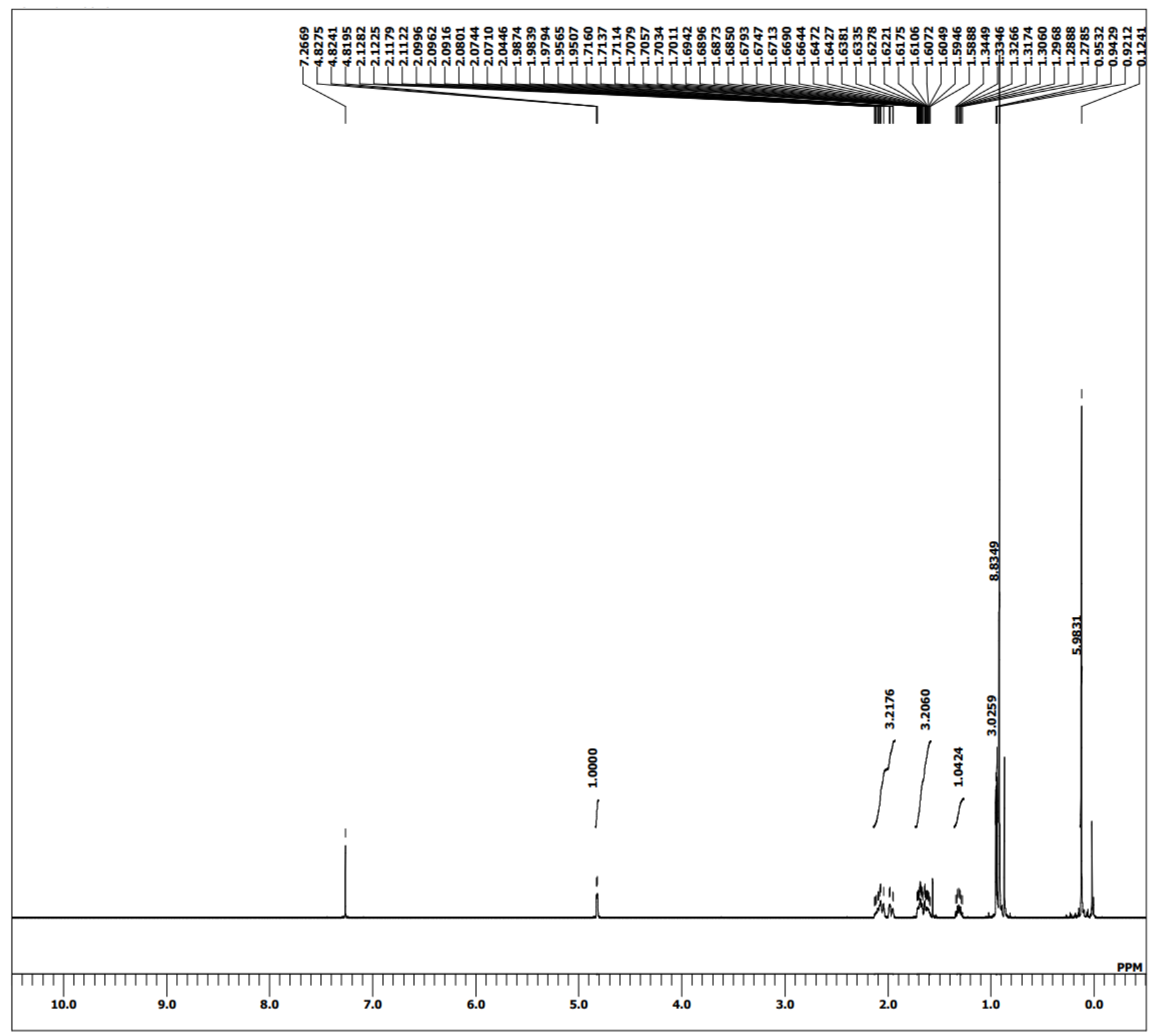


${ }^{1} \mathrm{H}$ NMR of $2 \mathrm{e}\left(500 \mathrm{MHz}, \mathrm{CDCl}_{3}\right)$
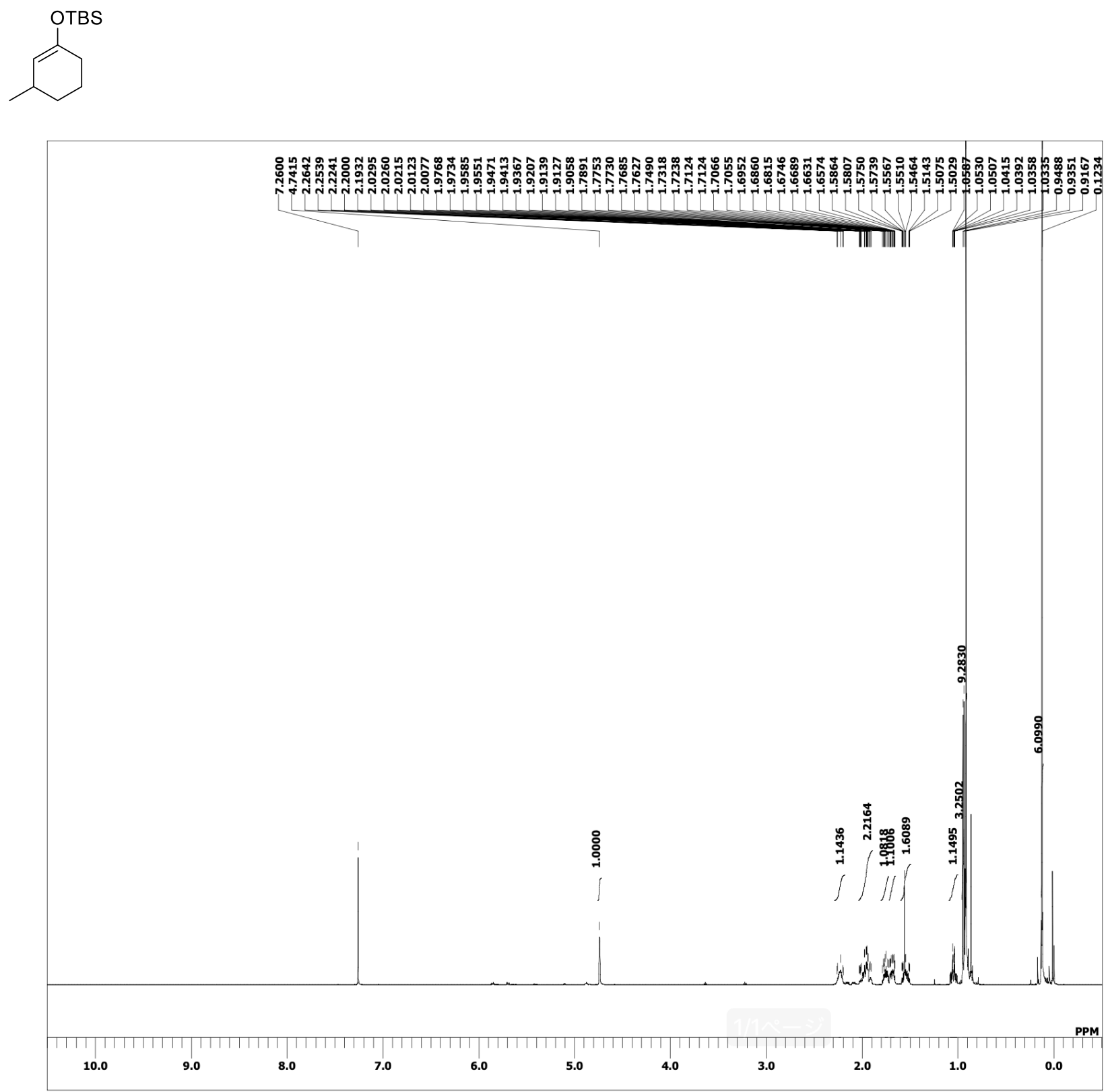
${ }^{13} \mathrm{C} \mathrm{NMR}$ of $2 \mathrm{e}\left(150 \mathrm{MHz}, \mathrm{CDCl}_{3}\right)$
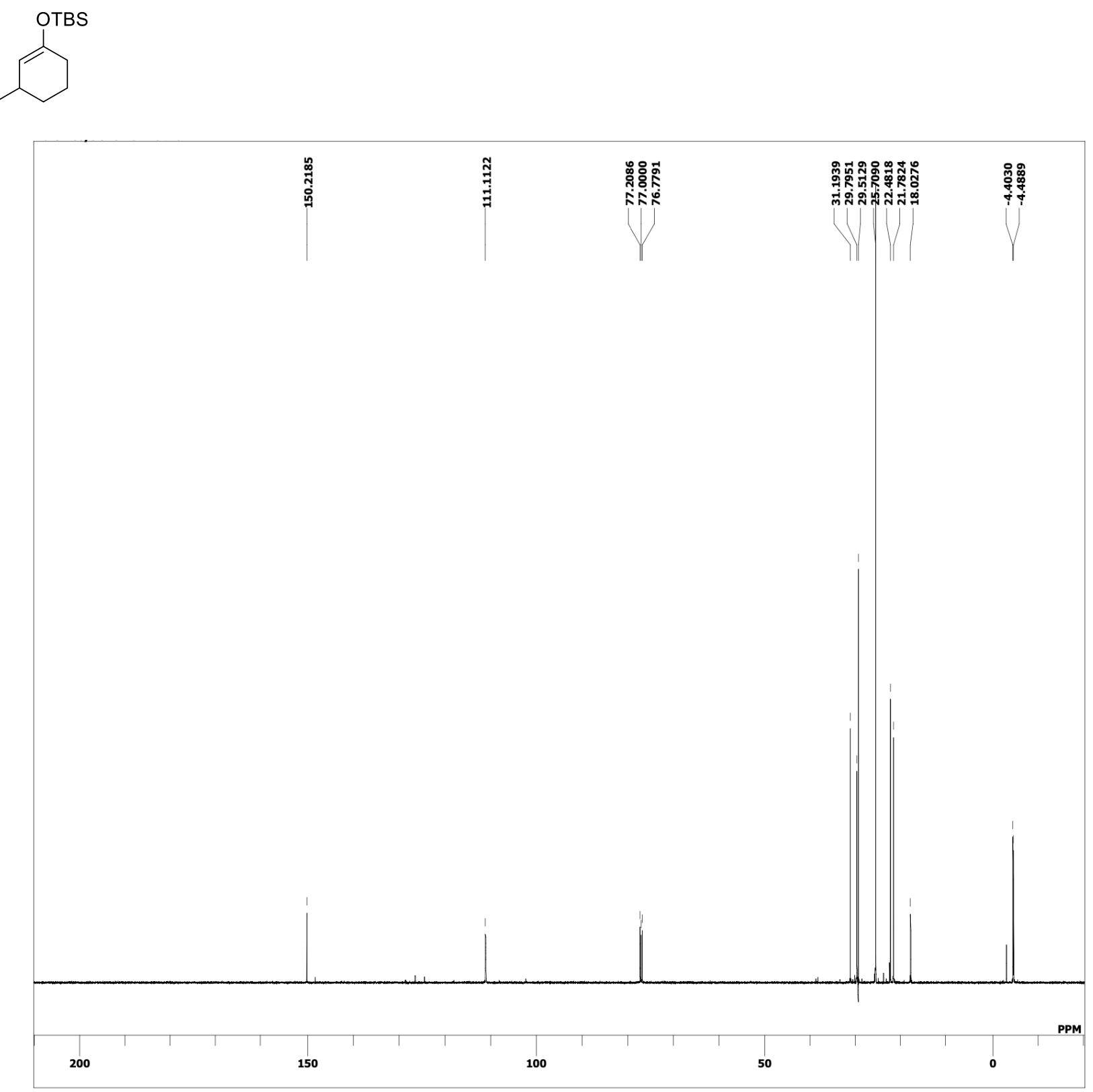
${ }^{1} \mathrm{H} \mathrm{NMR}$ of $\mathbf{2} \mathbf{f}\left(600 \mathrm{MHz}, \mathrm{CDCl}_{3}\right)$
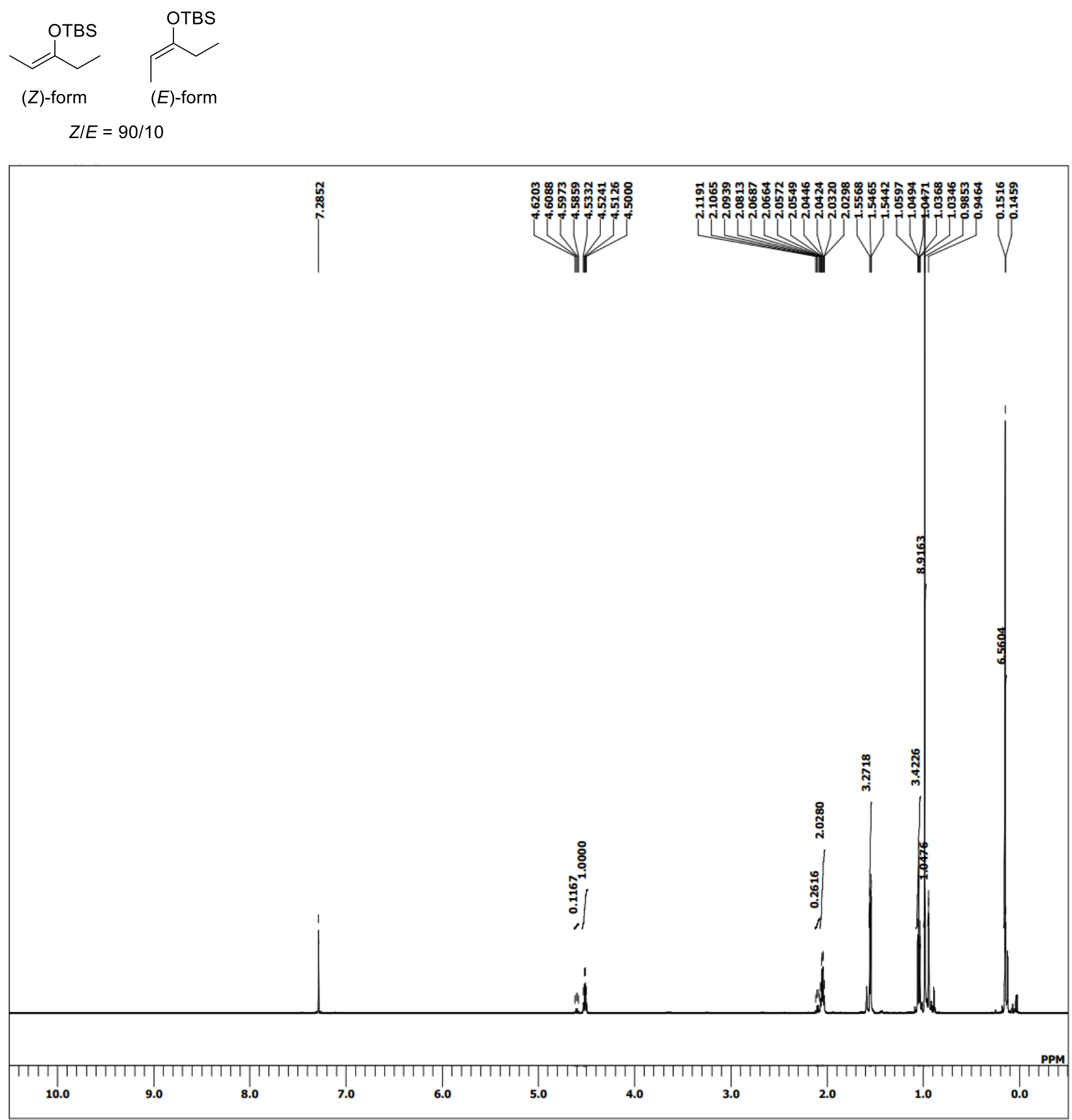
${ }^{1} \mathrm{H}$ NMR of $2 \mathbf{g}\left(600 \mathrm{MHz}, \mathrm{CDCl}_{3}\right)$
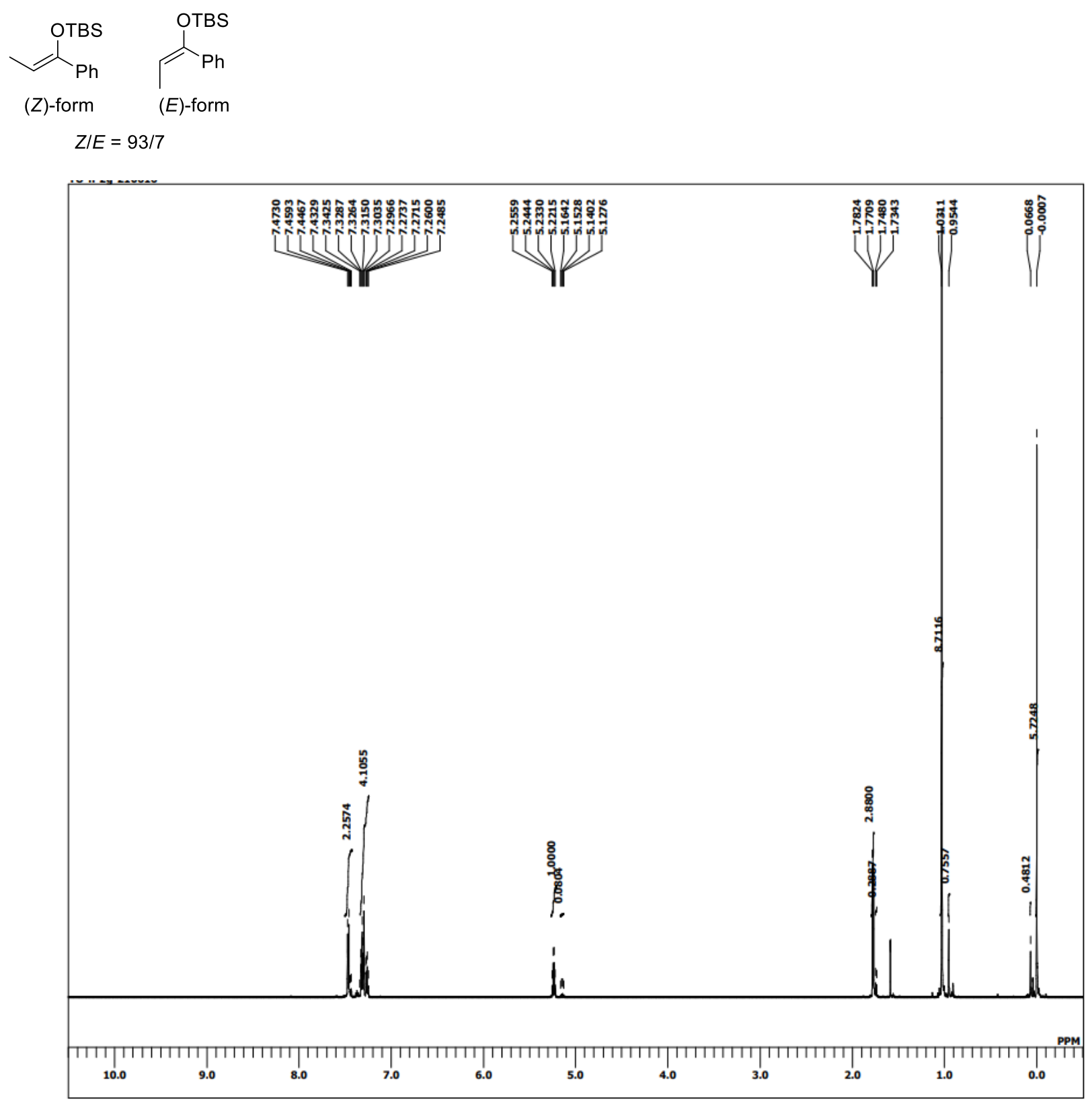
${ }^{1} \mathrm{H} \mathrm{NMR}$ of 3aa $\left(600 \mathrm{MHz}, \mathrm{CDCl}_{3}\right)$<smiles>O=C1CCCCC1CC(c1ccccc1)c1ccccc1</smiles>

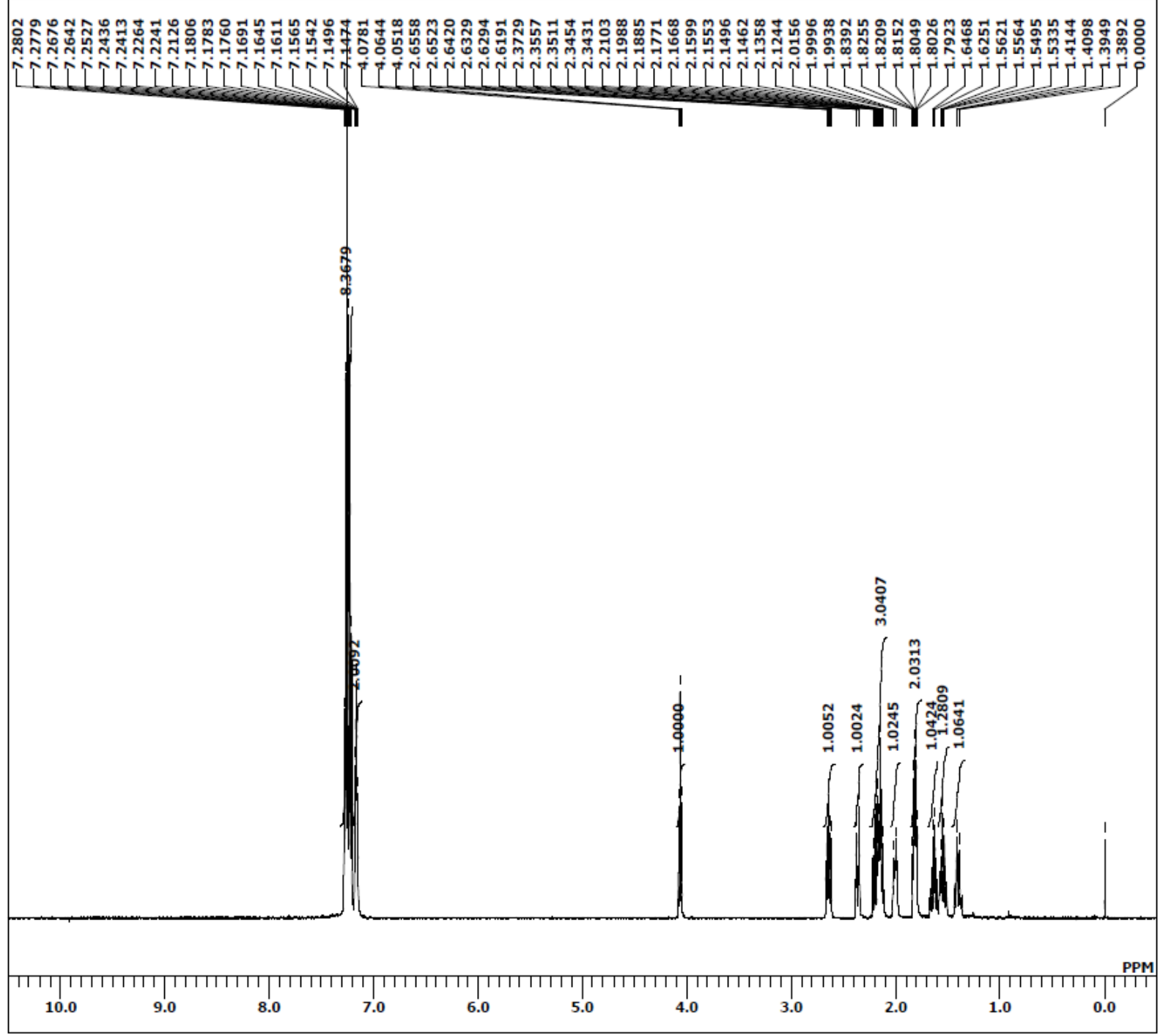


${ }^{13} \mathrm{C}$ NMR of 3aa $\left(150 \mathrm{MHz}, \mathrm{CDCl}_{3}\right)$

Ph

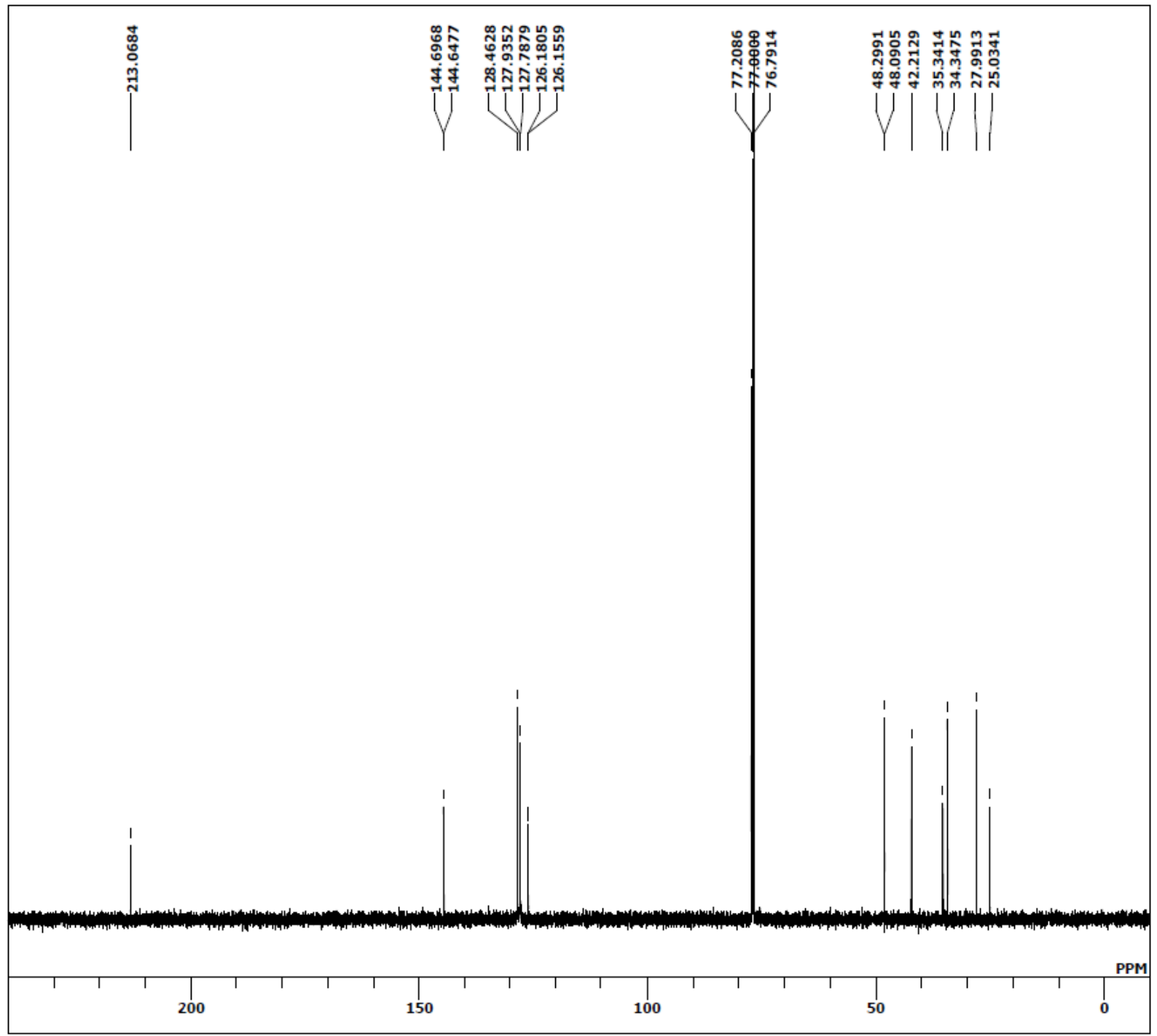


${ }^{1} \mathrm{H} \mathrm{NMR}$ of $\mathbf{3 b a}\left(600 \mathrm{MHz}, \mathrm{CDCl}_{3}\right)$<smiles>Cc1ccc(C(CC2CCCCC2=O)c2ccccc2)cc1</smiles>

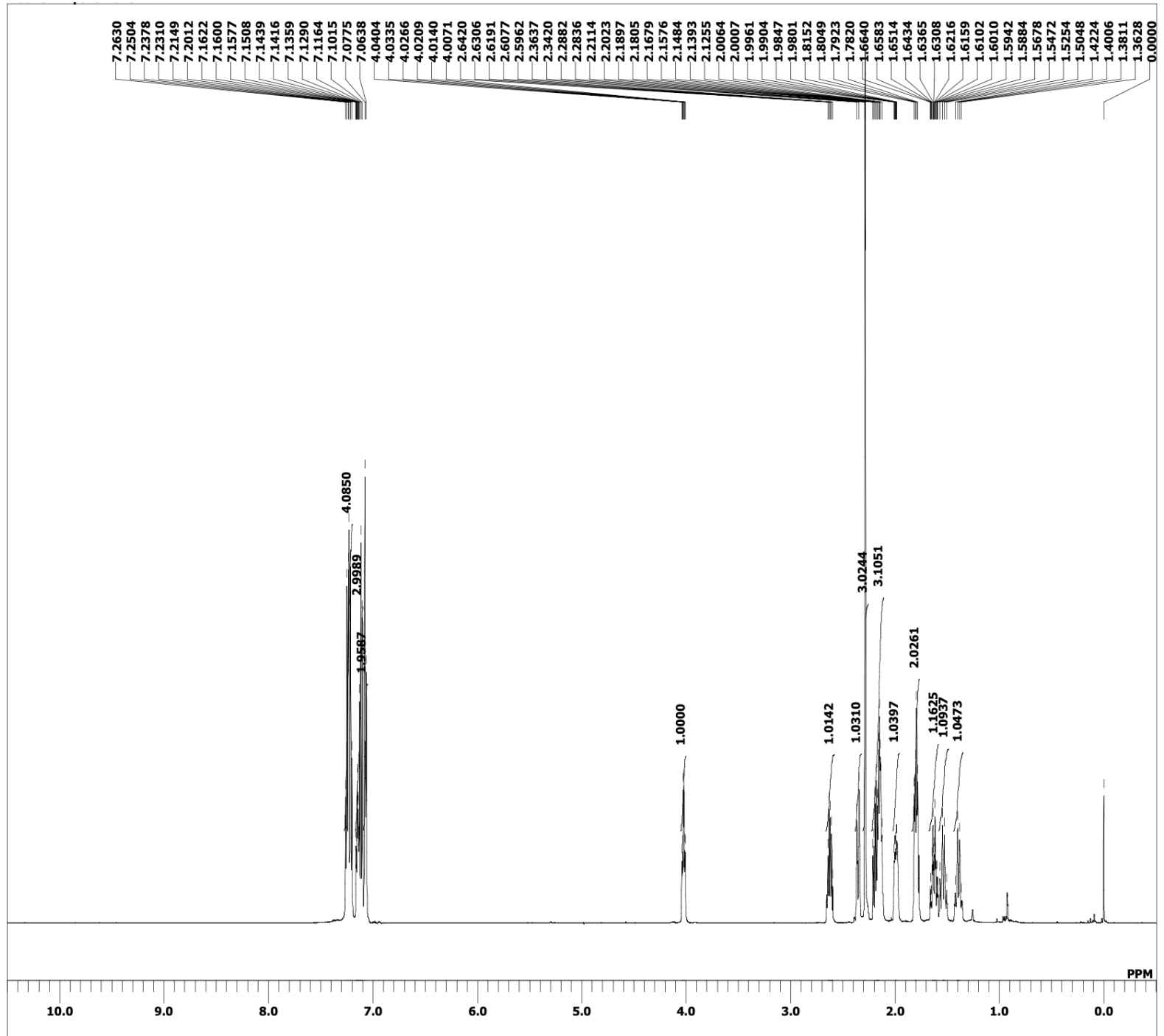


${ }^{13} \mathrm{C}$ NMR of 3 ba $\left(150 \mathrm{MHz}, \mathrm{CDCl}_{3}\right)$<smiles>[GeH2]</smiles>

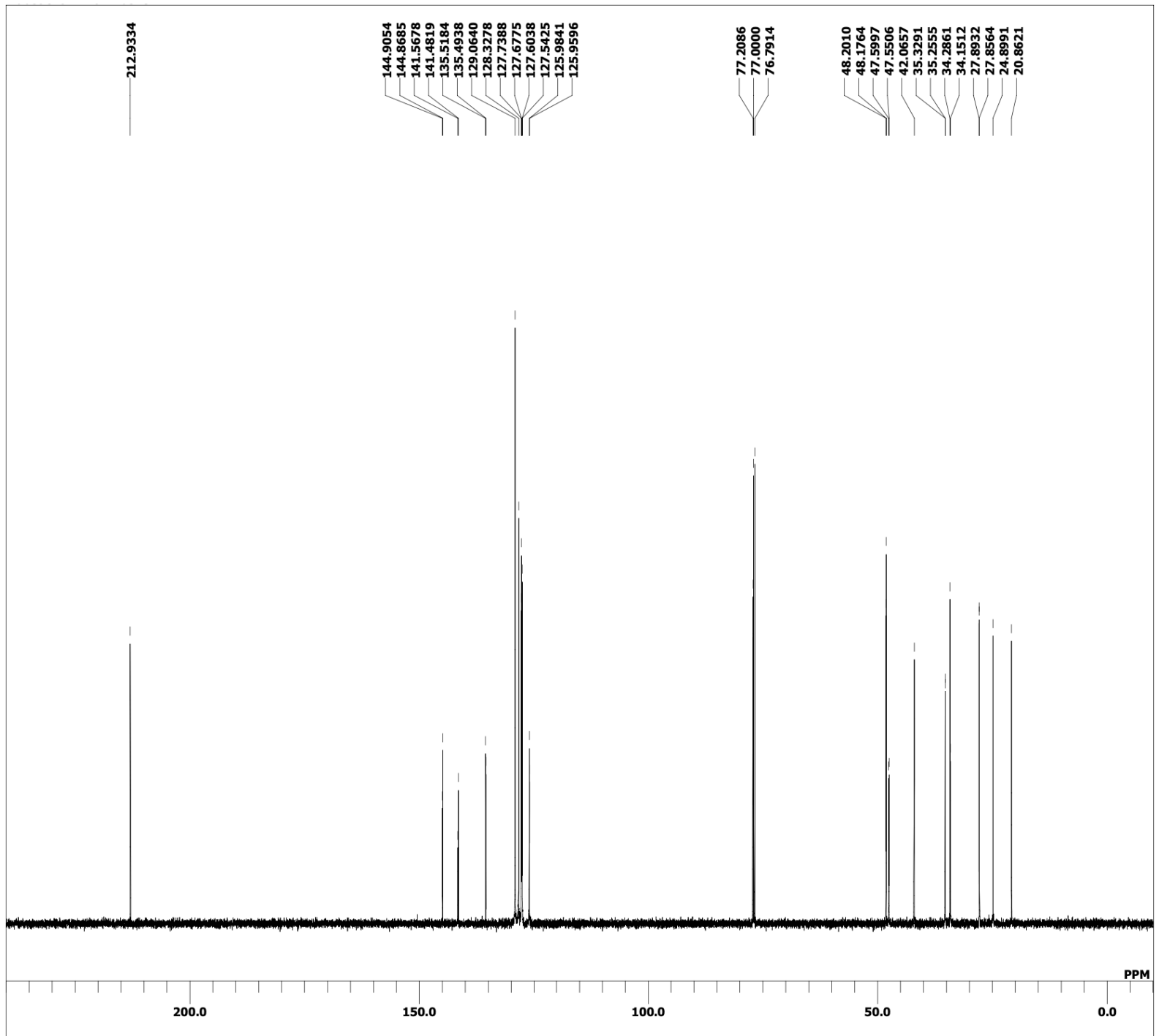


${ }^{1} \mathrm{H}$ NMR of $\mathbf{3 c a}\left(500 \mathrm{MHz}, \mathrm{CDCl}_{3}\right)$<smiles>Cc1ccccc1C(CC1CCCCC1=O)c1ccccc1</smiles>

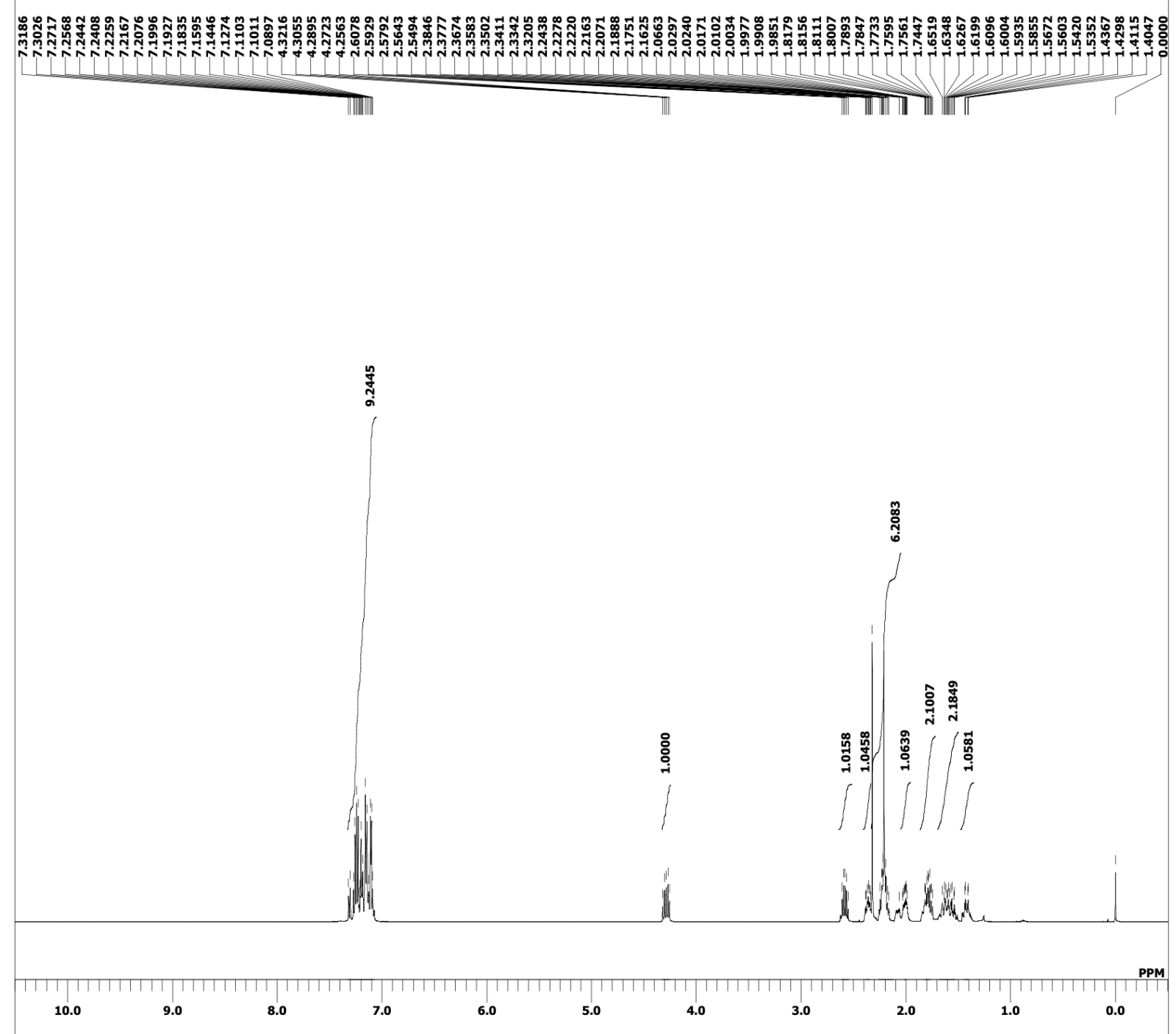


${ }^{13} \mathrm{C}$ NMR of $3 \mathbf{c a}\left(150 \mathrm{MHz}, \mathrm{CDCl}_{3}\right)$<smiles>Cc1ccccc1[C@H](CC1CCCCC1=O)c1ccccc1</smiles>

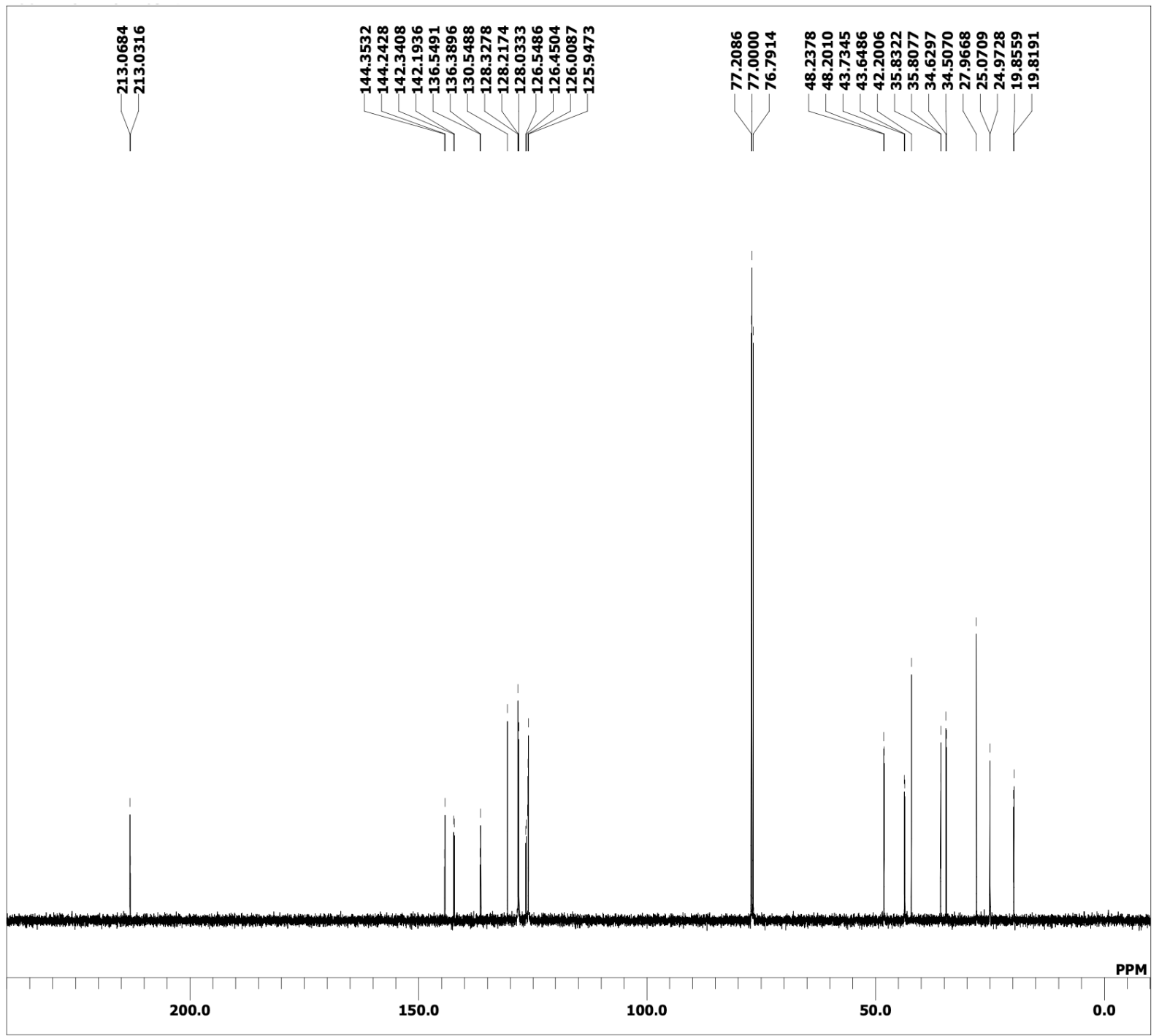


${ }^{1} \mathrm{H}$ NMR of 3da $\left(500 \mathrm{MHz}, \mathrm{CDCl}_{3}\right)$

$\mathrm{MeO}$<smiles>O=C1CCCCC1CC(c1ccccc1)c1ccccc1</smiles>

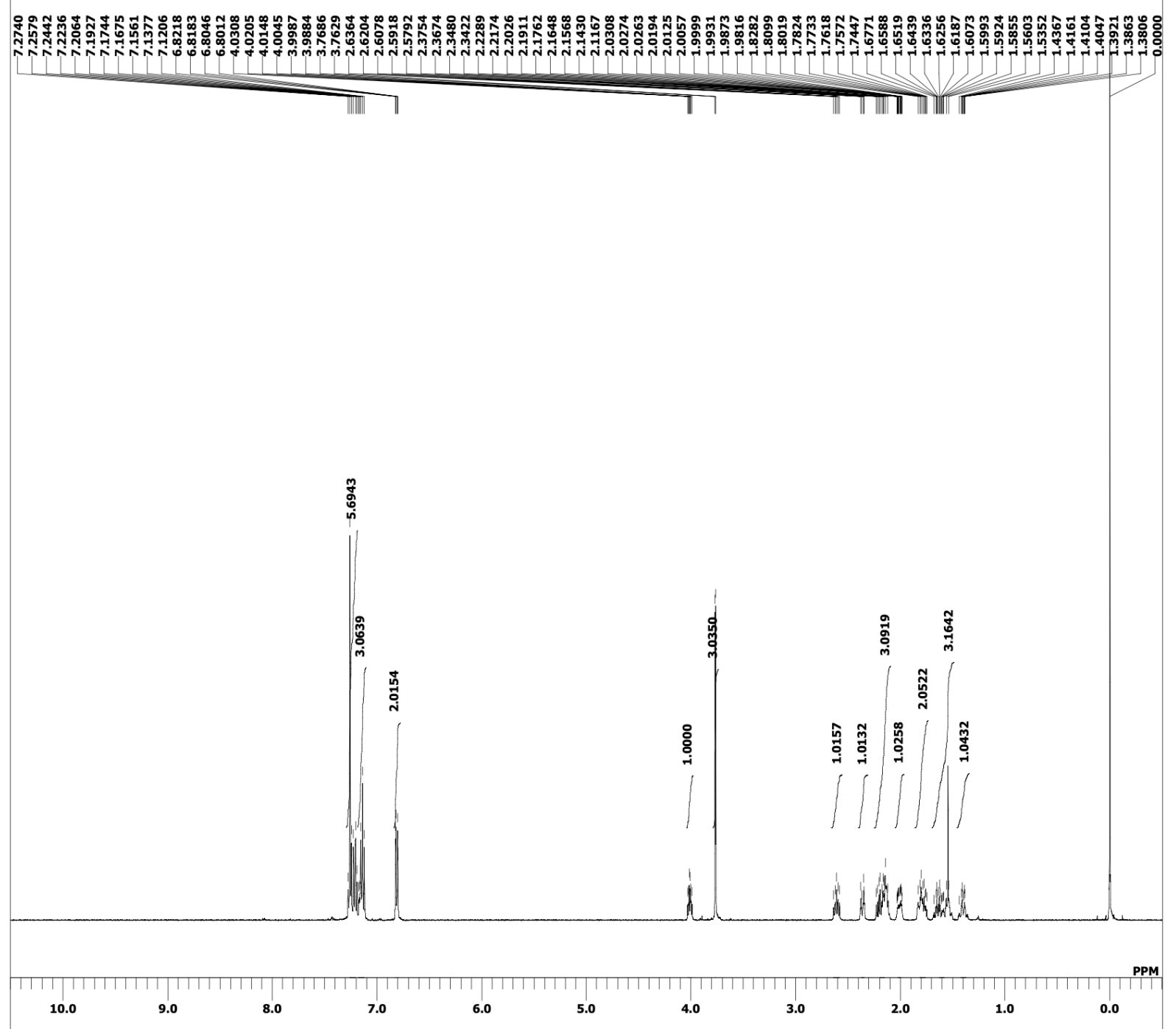


${ }^{13} \mathrm{C}$ NMR of 3da $\left(150 \mathrm{MHz}, \mathrm{CDCl}_{3}\right)$

$\mathrm{MeO}$<smiles>O=C1CCCCC1CC(c1ccccc1)c1ccccc1</smiles>

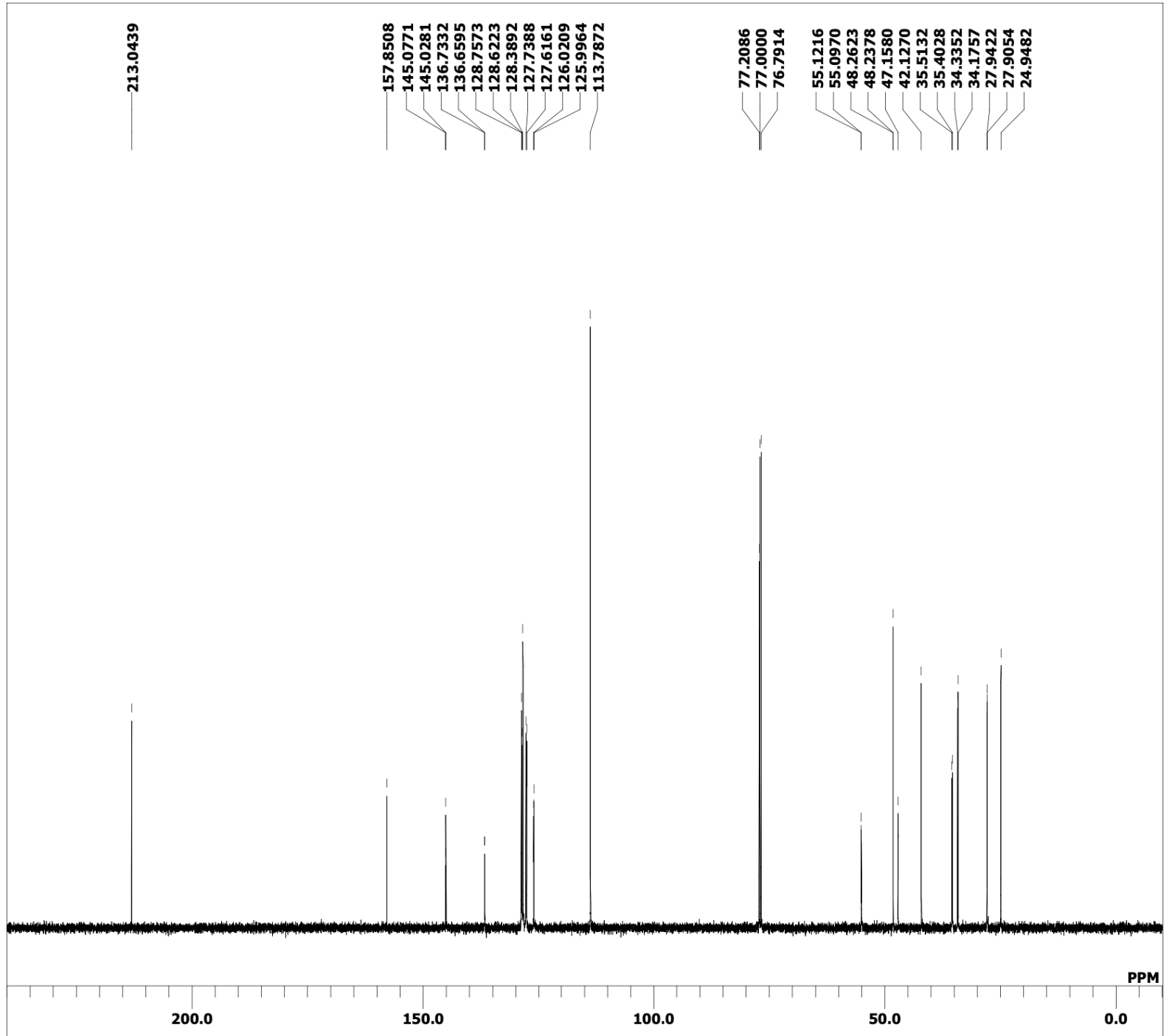


${ }^{1} \mathrm{H}$ NMR of 3 ea $\left(500 \mathrm{MHz}, \mathrm{CDCl}_{3}\right)$<smiles>O=C1CCCCC1CC(c1ccccc1)c1ccc(Br)cc1</smiles>

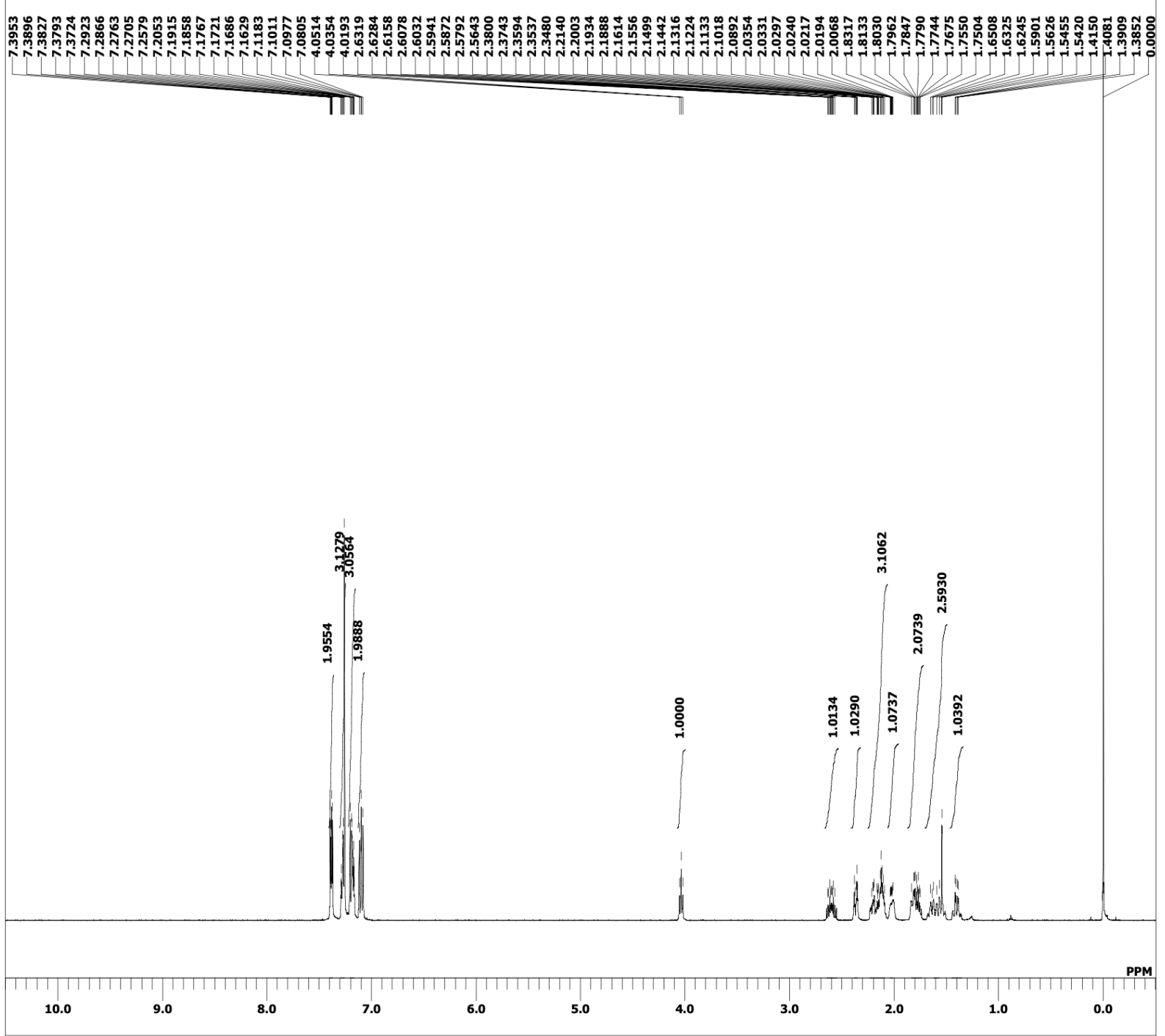


${ }^{13} \mathrm{C}$ NMR of 3ea $\left(150 \mathrm{MHz}, \mathrm{CDCl}_{3}\right)$

(O)

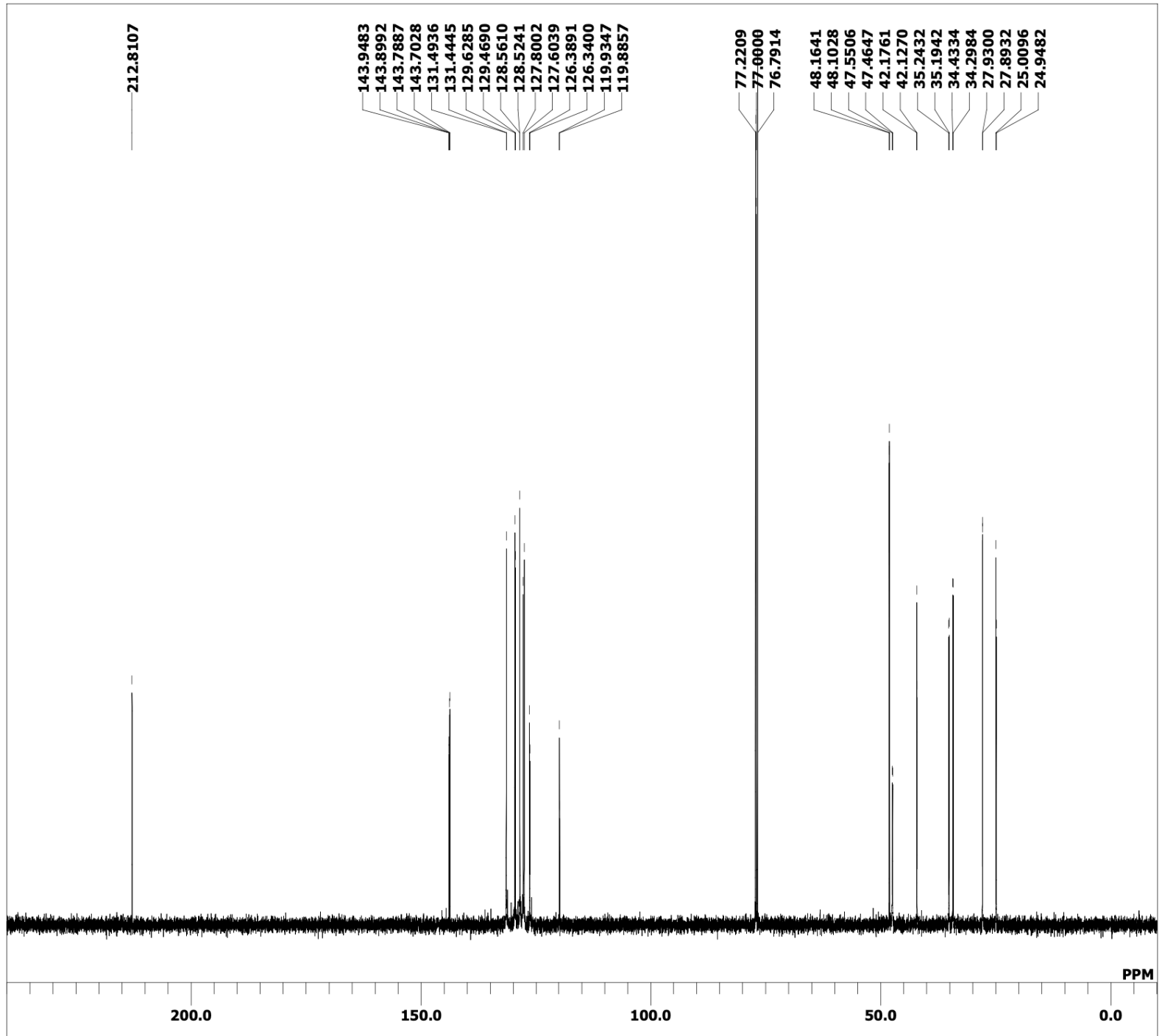


${ }^{1} \mathrm{H}$ NMR of $\mathbf{3 f a}\left(500 \mathrm{MHz}, \mathrm{CDCl}_{3}\right)$<smiles>COc1ccc(C(CC2CCCCC2=O)c2ccc(OC)cc2)cc1</smiles>

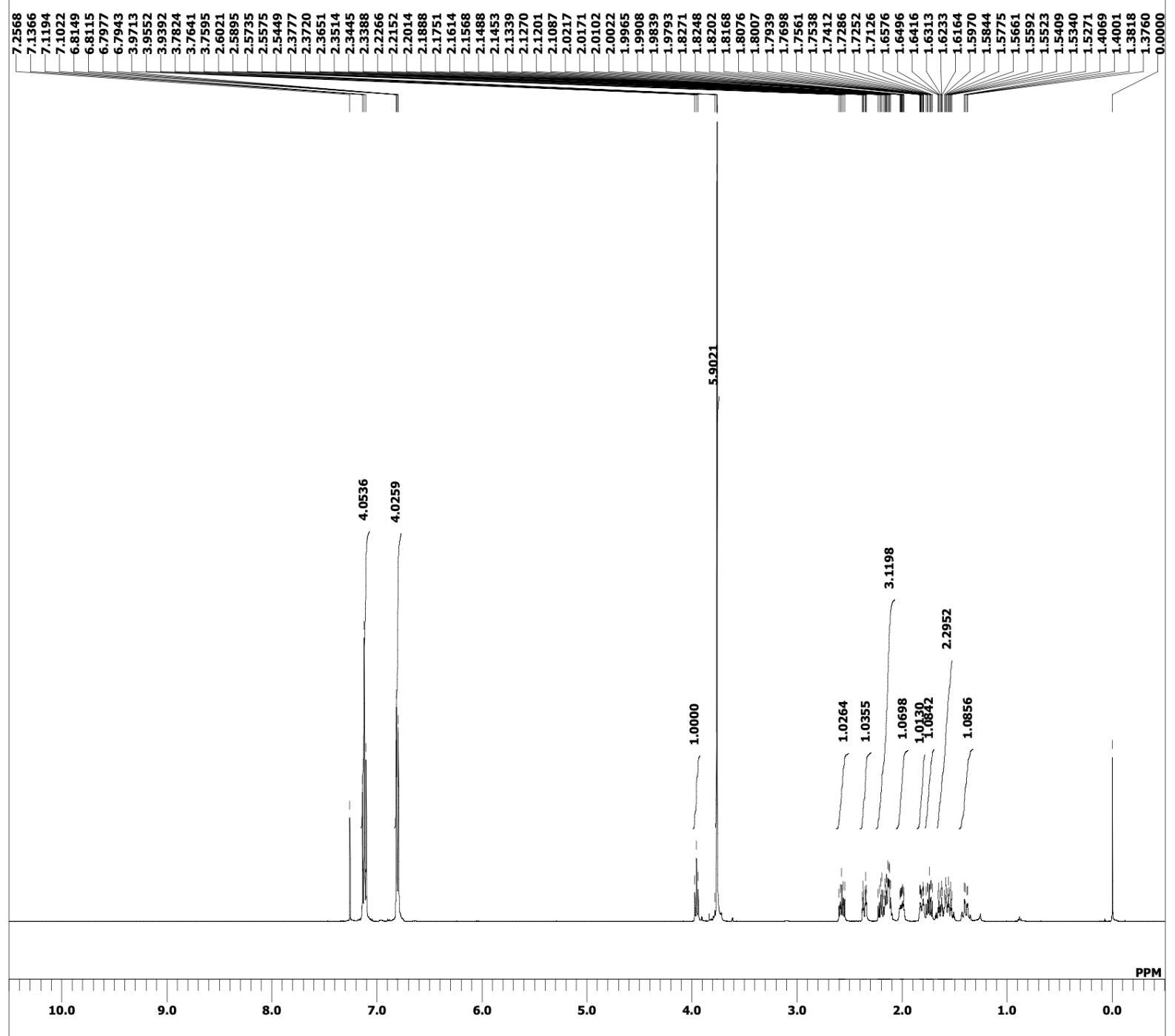


${ }^{13} \mathrm{C}$ NMR of $\mathbf{3 f a}\left(125 \mathrm{MHz}, \mathrm{CDCl}_{3}\right)$<smiles>COc1ccc(C(CC2CCCCC2=O)c2ccc(OC)cc2)cc1</smiles>

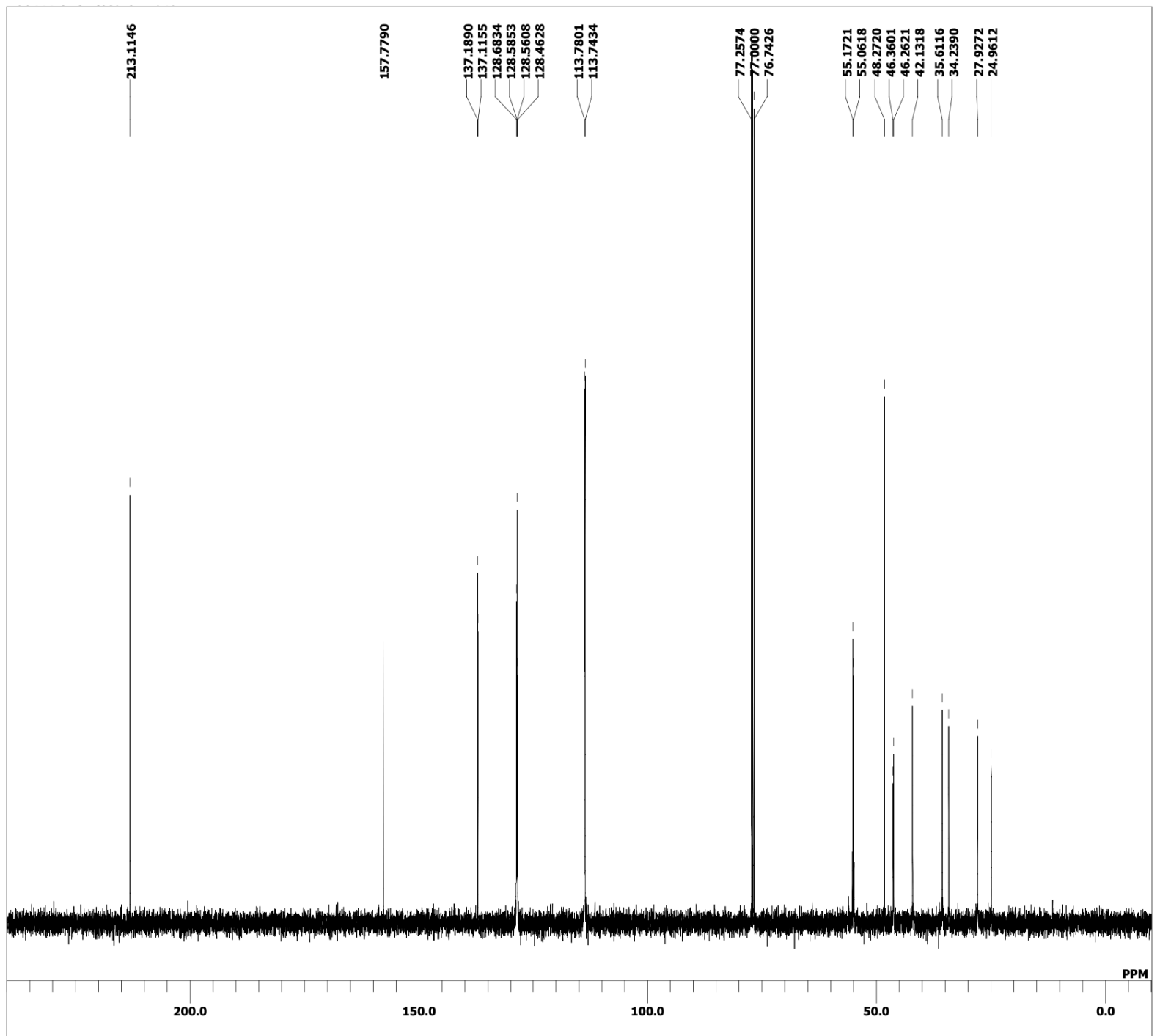


${ }^{1} \mathrm{H}$ NMR of 3 ga $\left(500 \mathrm{MHz}, \mathrm{CDCl}_{3}\right)$<smiles>O=C1CCCCC1CC(c1ccc(F)cc1)c1ccc(F)cc1</smiles>

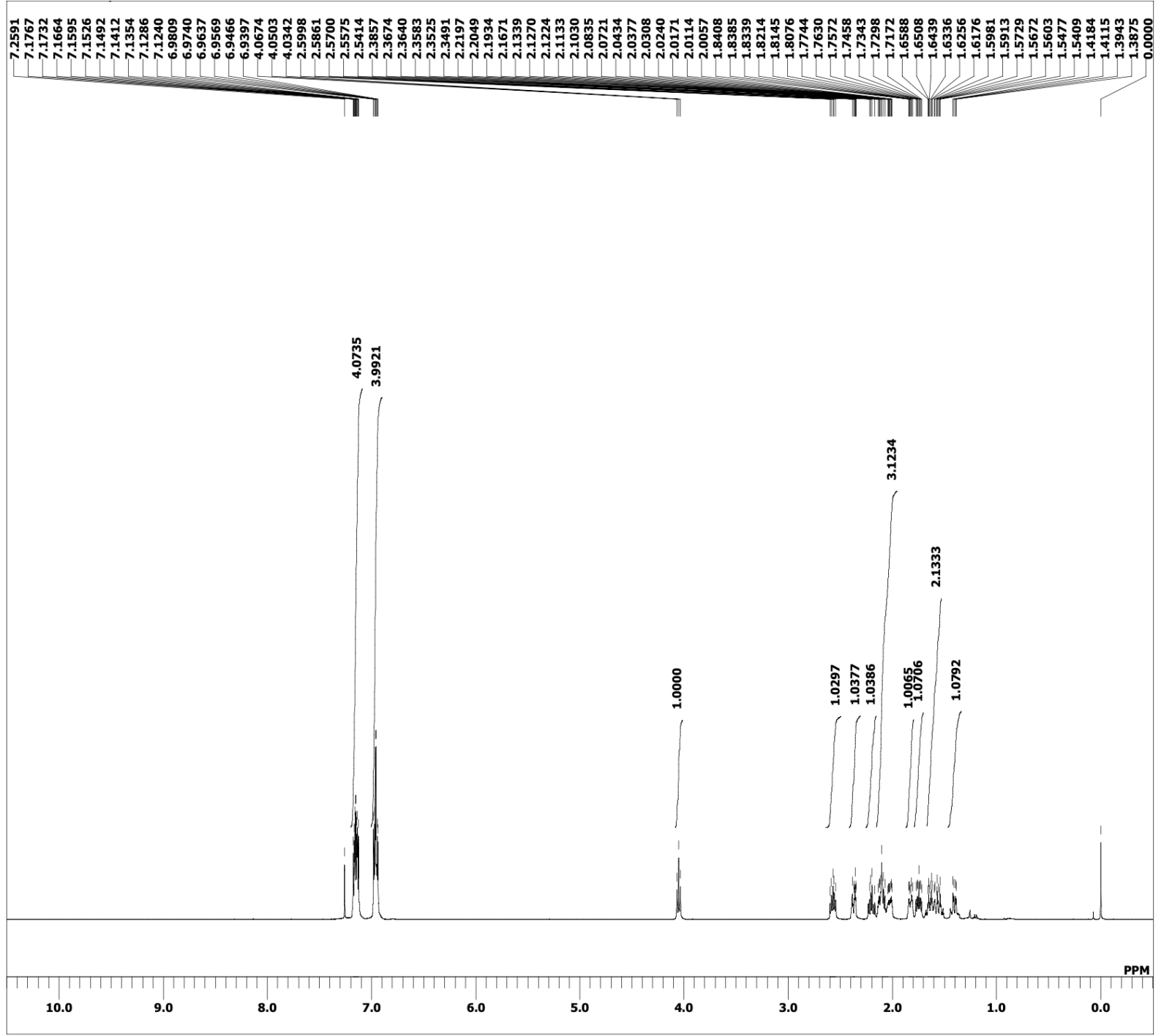


${ }^{13} \mathrm{C} \mathrm{NMR}$ of $3 g a\left(150 \mathrm{MHz}, \mathrm{CDCl}_{3}\right)$

(1)

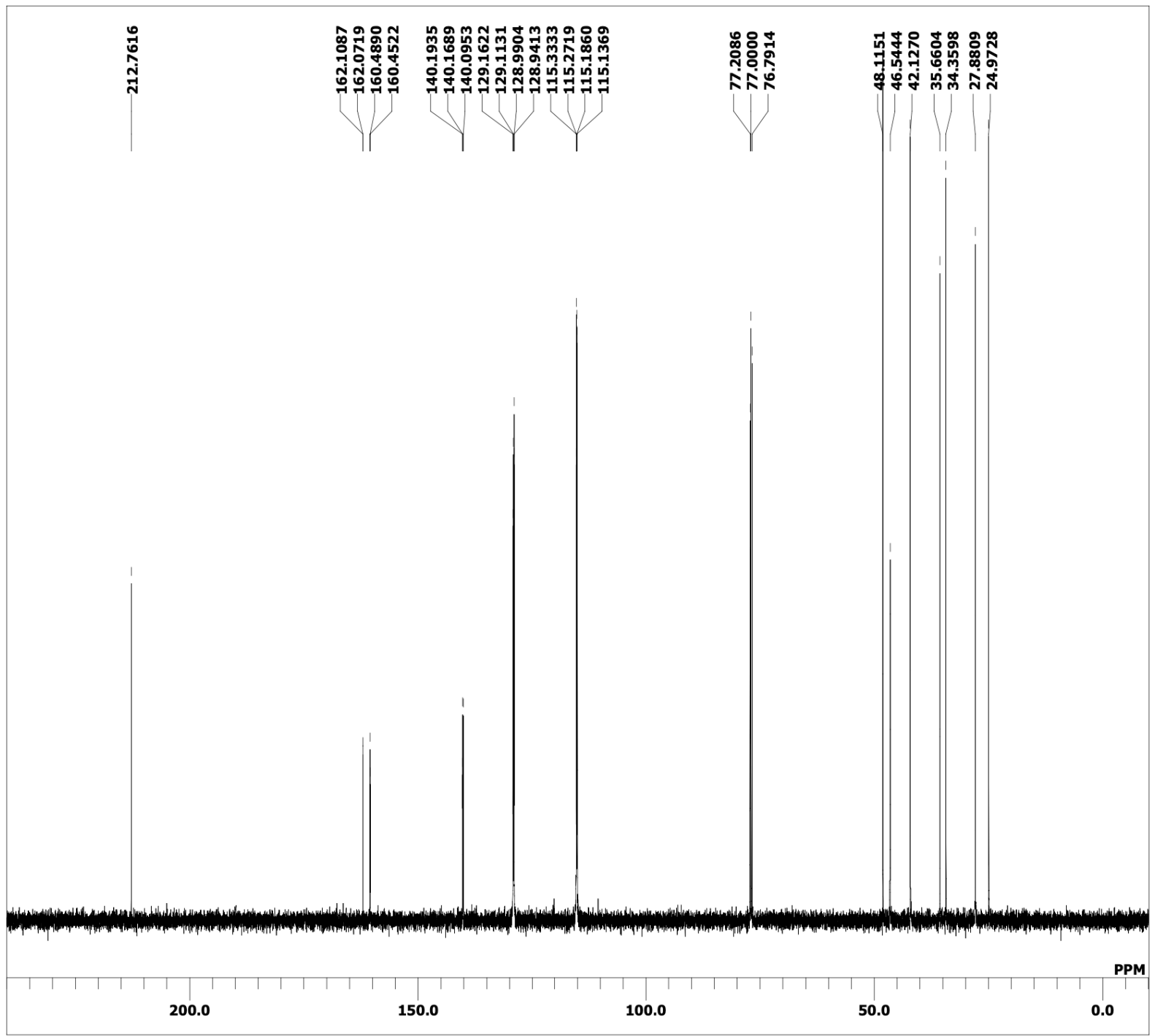


${ }^{19} \mathrm{~F}$ NMR of 3 ga $\left(565 \mathrm{MHz}, \mathrm{CDCl}_{3}\right)$<smiles>O=C1CCCCC1CC(c1ccc(F)cc1)c1ccc(F)cc1</smiles>

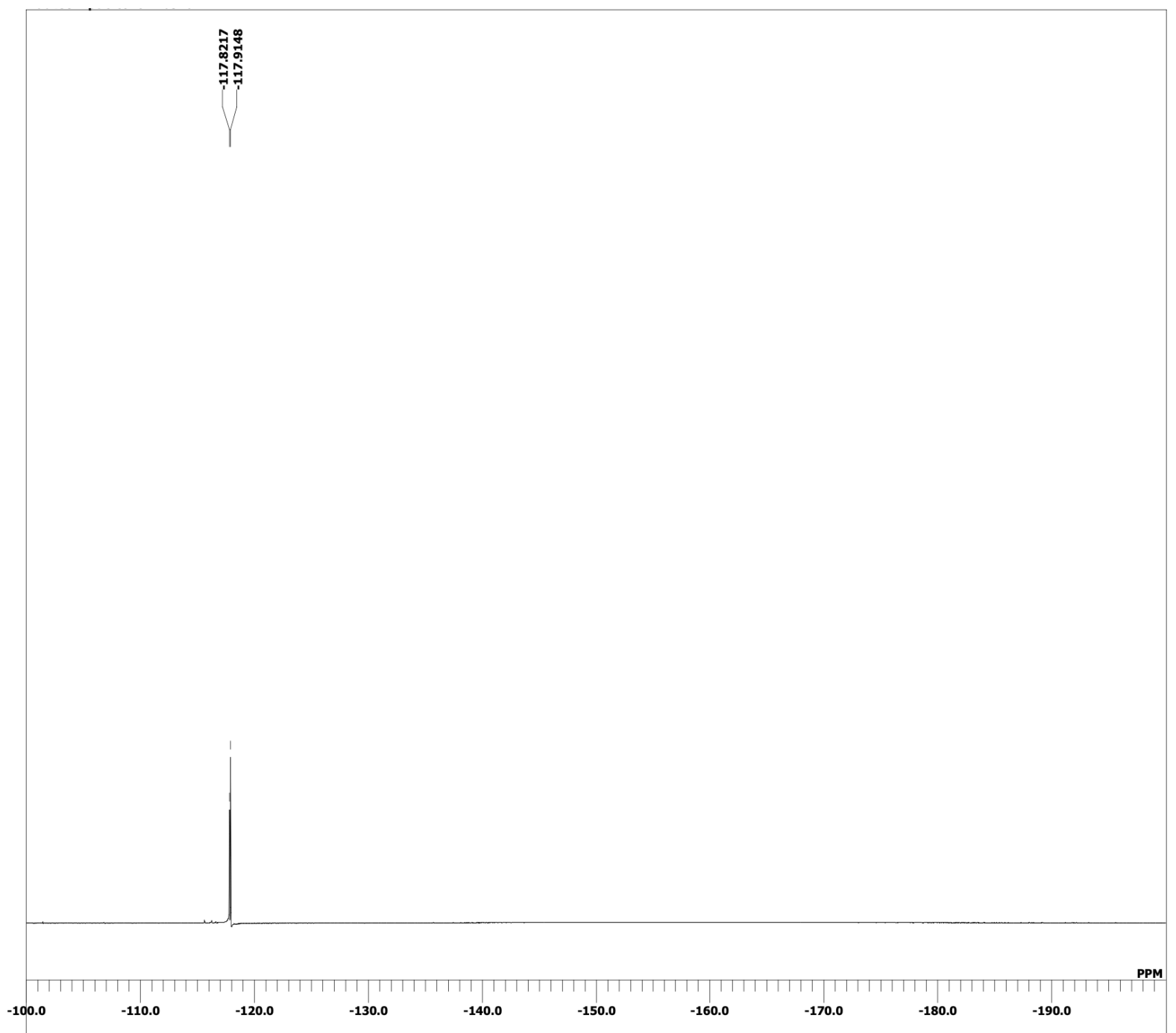


${ }^{1} \mathrm{H}$ NMR of 3 ha $\left(600 \mathrm{MHz}, \mathrm{CDCl}_{3}\right)$<smiles>O=C1CCCCC1CCc1ccccc1</smiles>

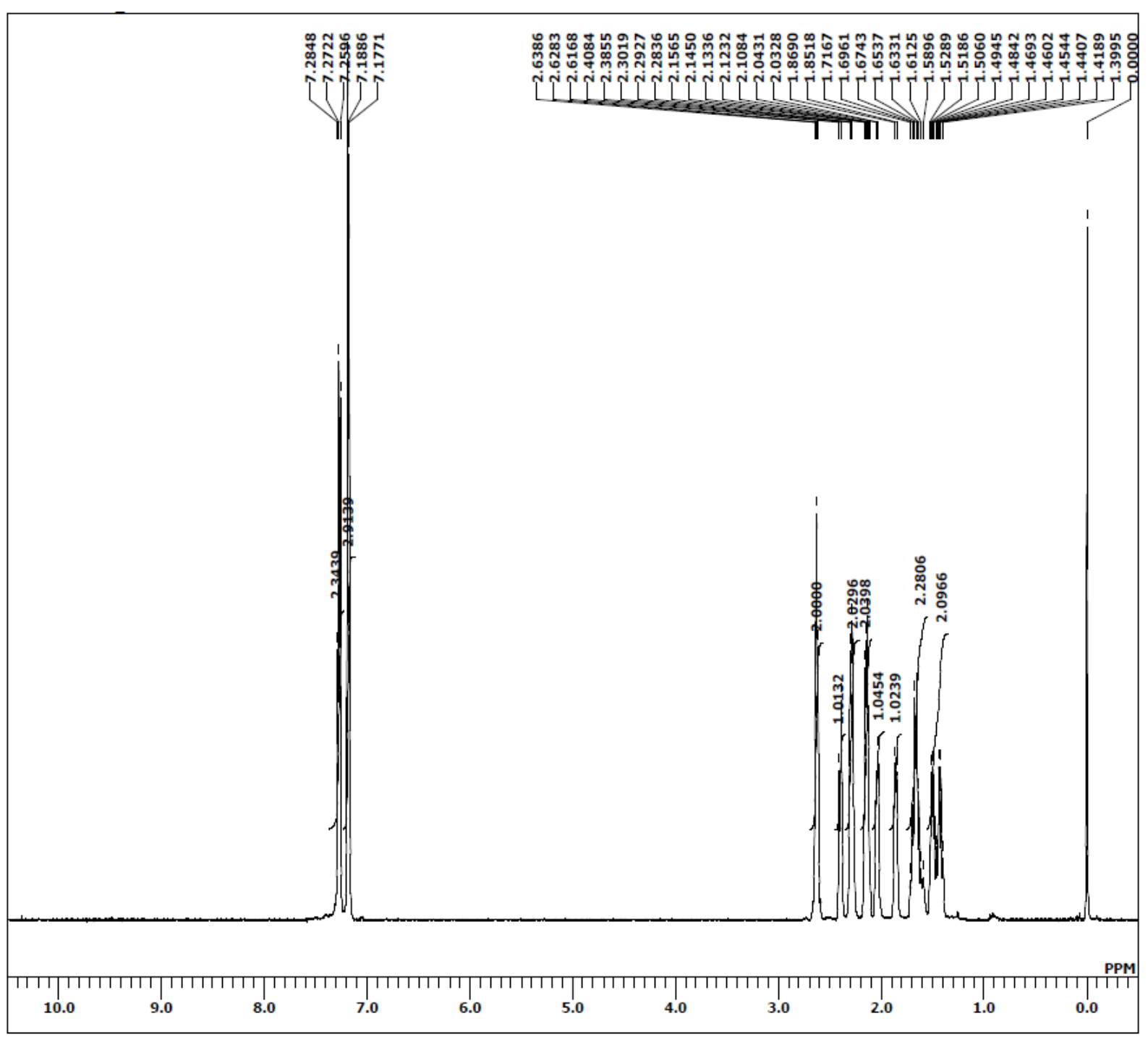


${ }^{13} \mathrm{C}$ NMR of 3 ha $\left(150 \mathrm{MHz}, \mathrm{CDCl}_{3}\right)$<smiles>O=C1CCCCC1CCc1ccccc1</smiles>




${ }^{1} \mathrm{H}$ NMR of 3 ia $\left(600 \mathrm{MHz}, \mathrm{CDCl}_{3}\right)$<smiles>Cc1ccc(CCC2CCCCC2=O)cc1</smiles>

ถ⿻ำกำำำ

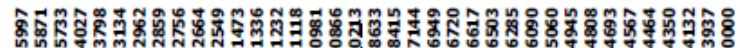

i个j

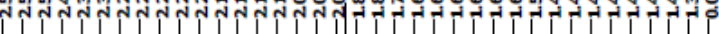

Tाก | | IIII


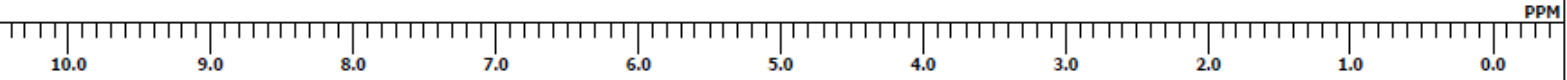


${ }^{13} \mathrm{C}$ NMR of 3ia $\left(150 \mathrm{MHz}, \mathrm{CDCl}_{3}\right)$

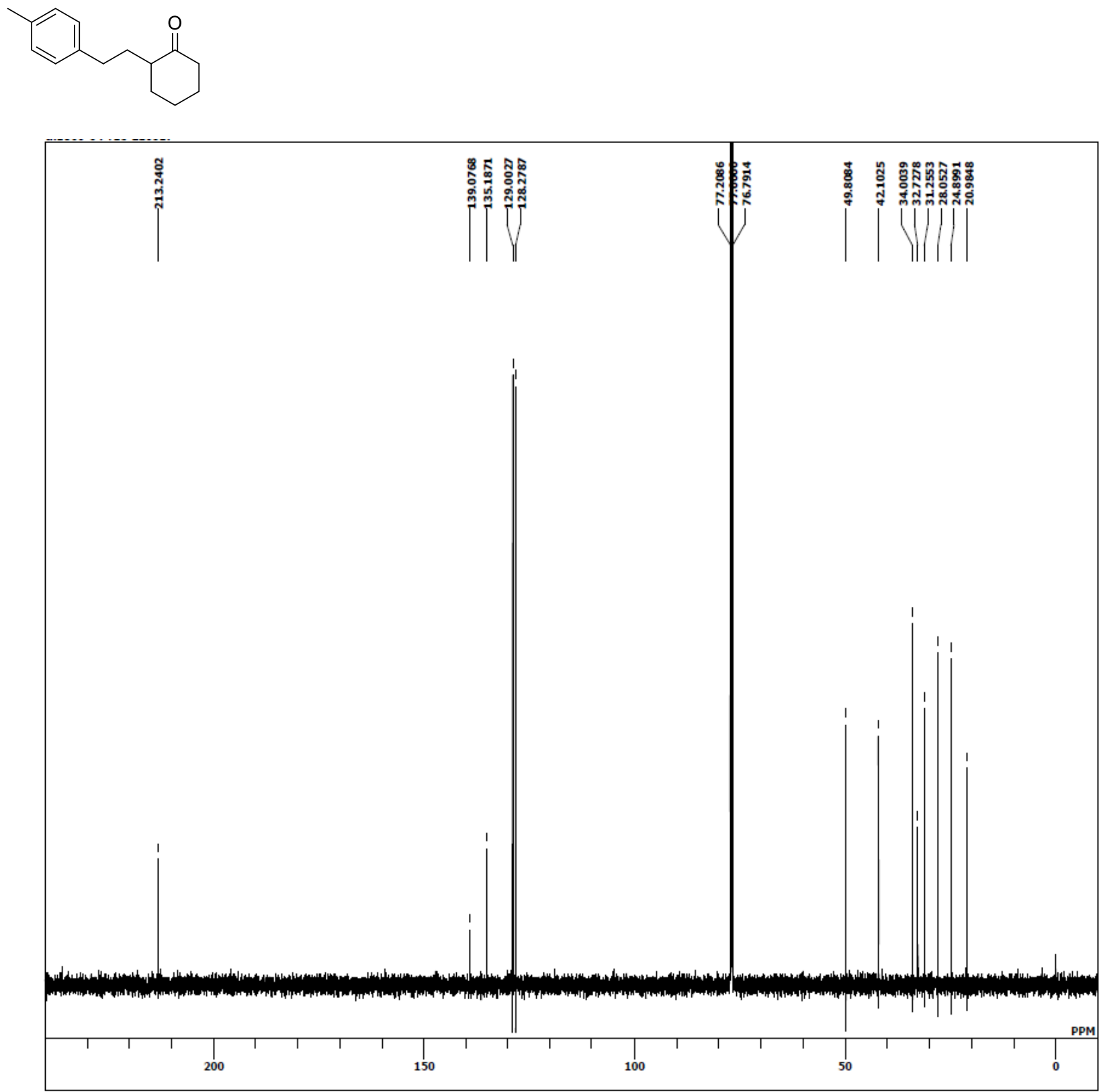


${ }^{1} \mathrm{H}$ NMR of $\mathbf{3 j a}\left(600 \mathrm{MHz}, \mathrm{CDCl}_{3}\right)$
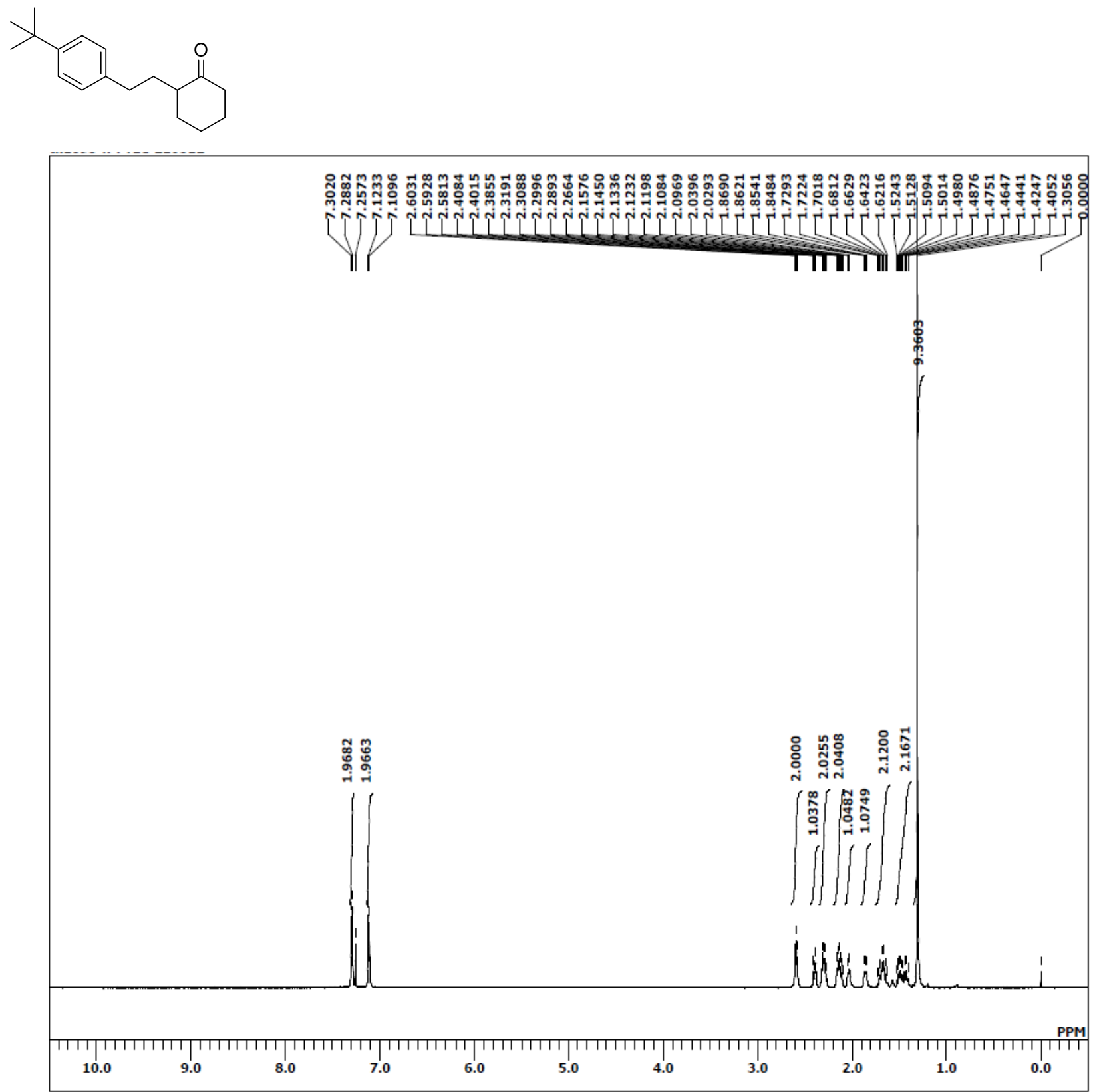
${ }^{13} \mathrm{C}$ NMR of 3ja $\left(150 \mathrm{MHz}, \mathrm{CDCl}_{3}\right)$

$>1$

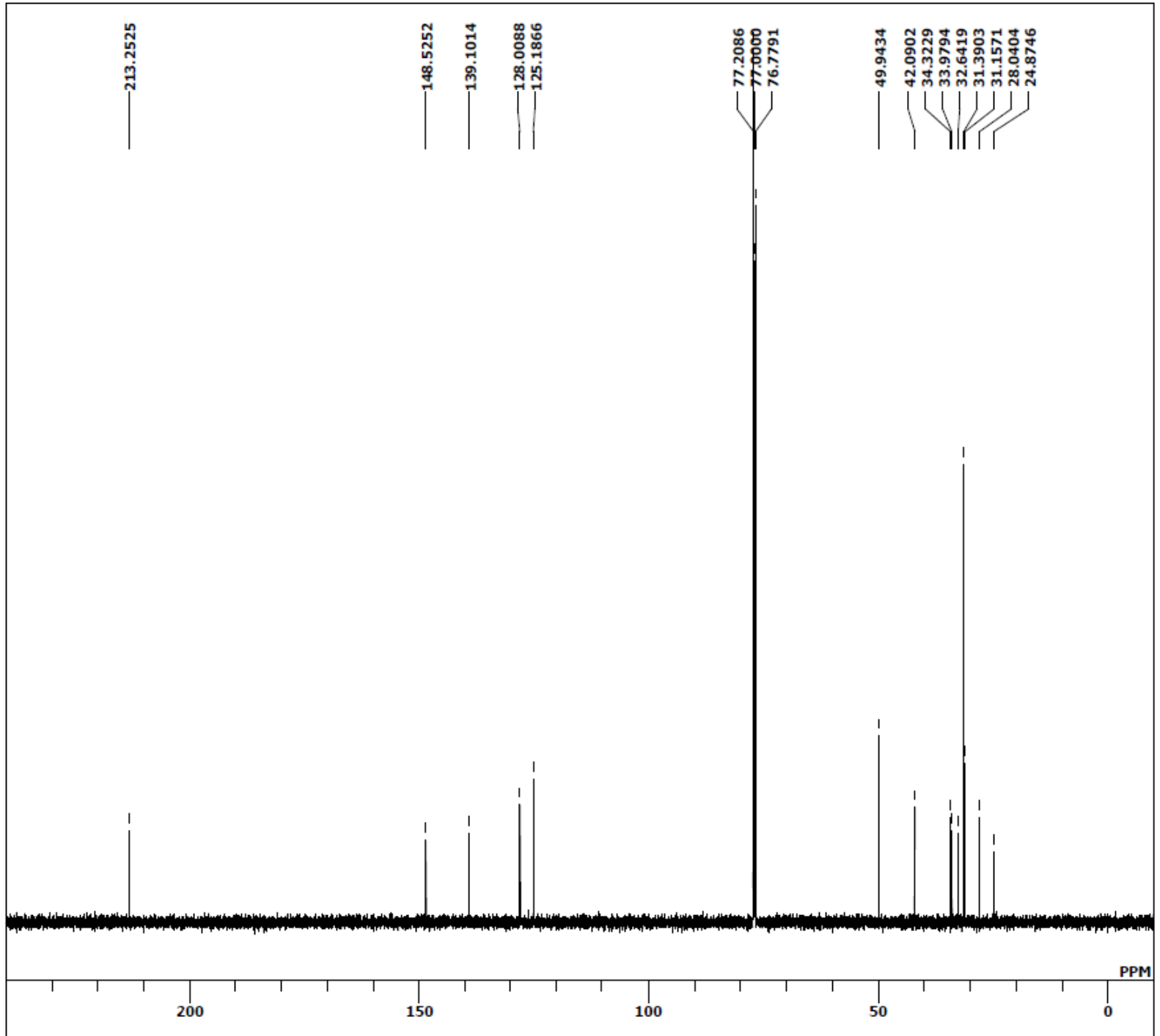


${ }^{1} \mathrm{H}$ NMR of $\mathbf{3 k a}\left(600 \mathrm{MHz}, \mathrm{CDCl}_{3}\right)$
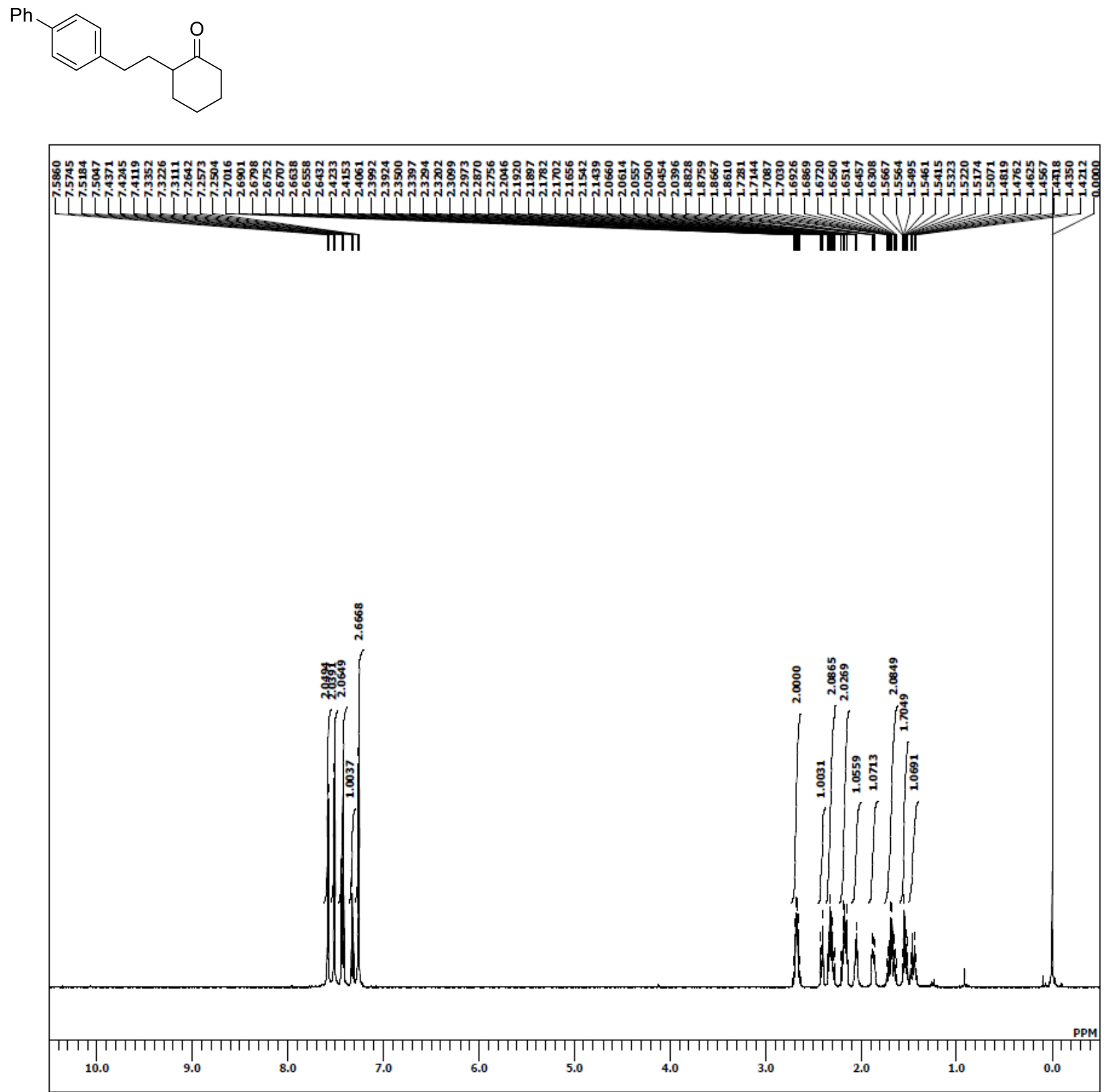
${ }^{13} \mathrm{C}$ NMR of 3ka $\left(150 \mathrm{MHz}, \mathrm{CDCl}_{3}\right)$




${ }^{1} \mathrm{H}$ NMR of 3la $\left(600 \mathrm{MHz}, \mathrm{CDCl}_{3}\right)$

$\mathrm{MeO}$<smiles>Cc1ccc(CCC2CCCCC2=O)cc1</smiles>

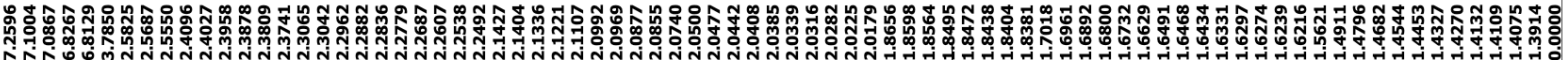

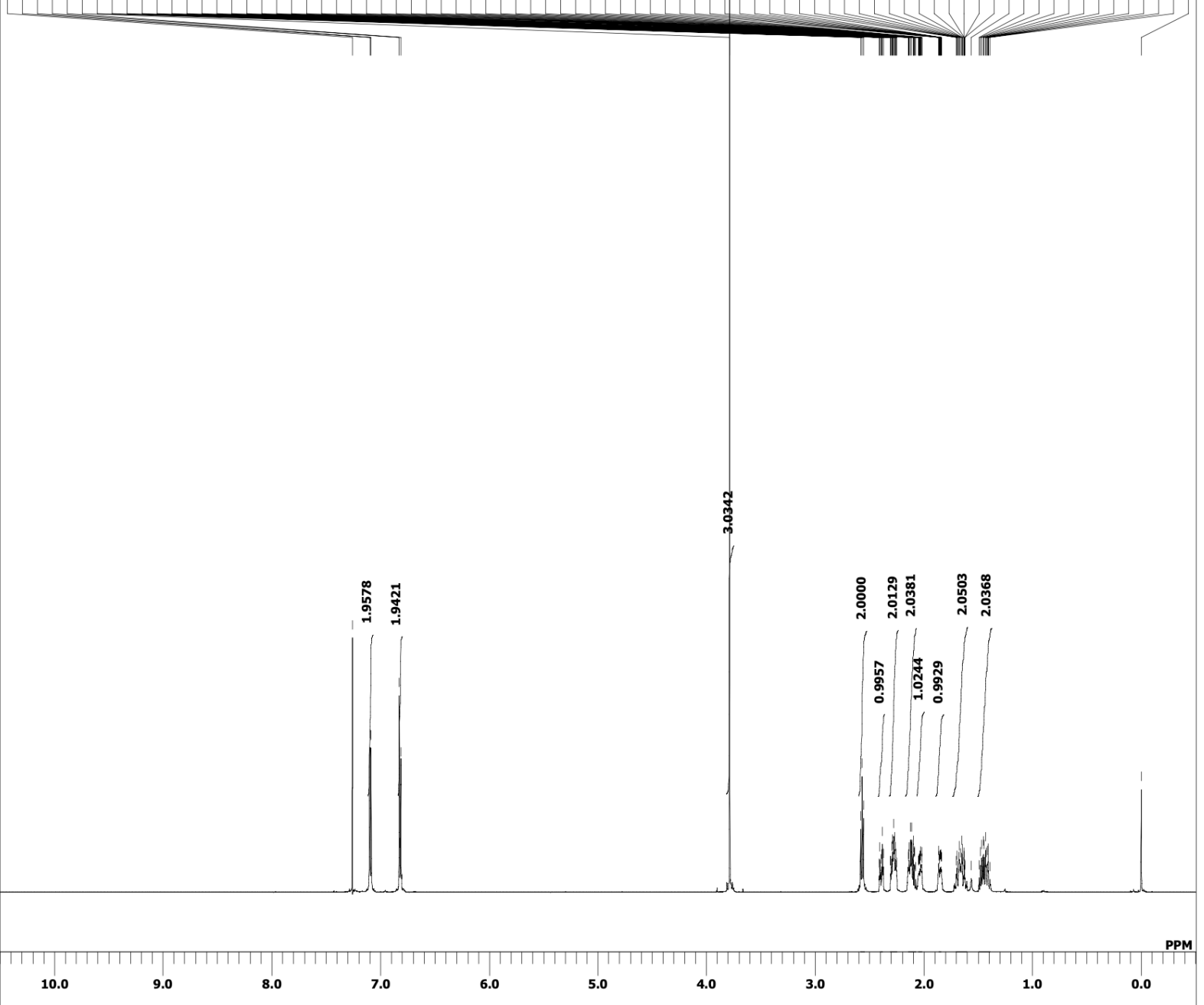


${ }^{13} \mathrm{C}$ NMR of 3la $\left(150 \mathrm{MHz}, \mathrm{CDCl}_{3}\right)$

$\mathrm{MeO}$<smiles>Cc1ccc(CCC2CCCCC2=O)cc1</smiles>

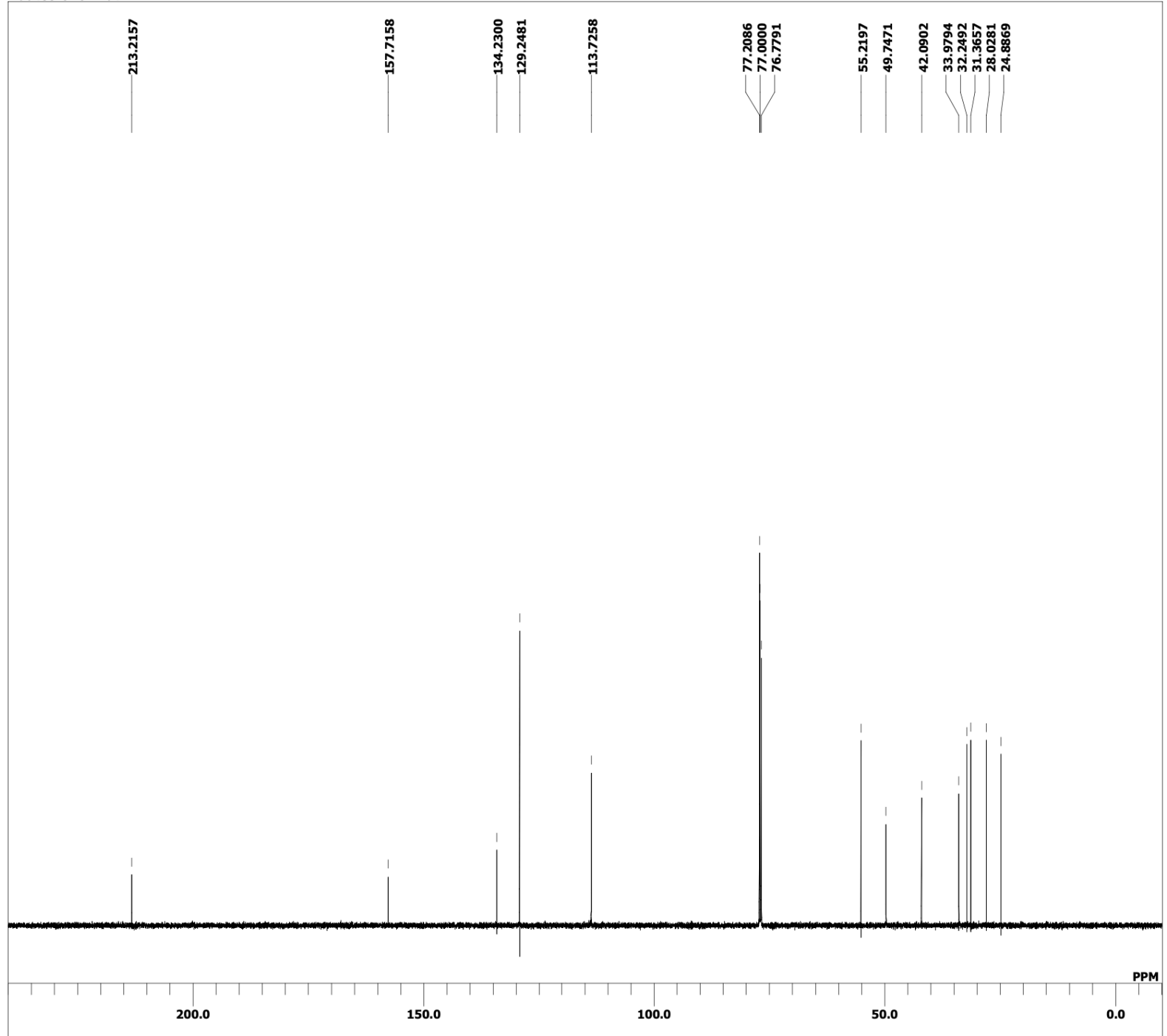


${ }^{1} \mathrm{H}$ NMR of $\mathbf{3 m a}\left(600 \mathrm{MHz}, \mathrm{CDCl}_{3}\right)$<smiles>COc1cccc(CCC2CCCCC2=O)c1</smiles>

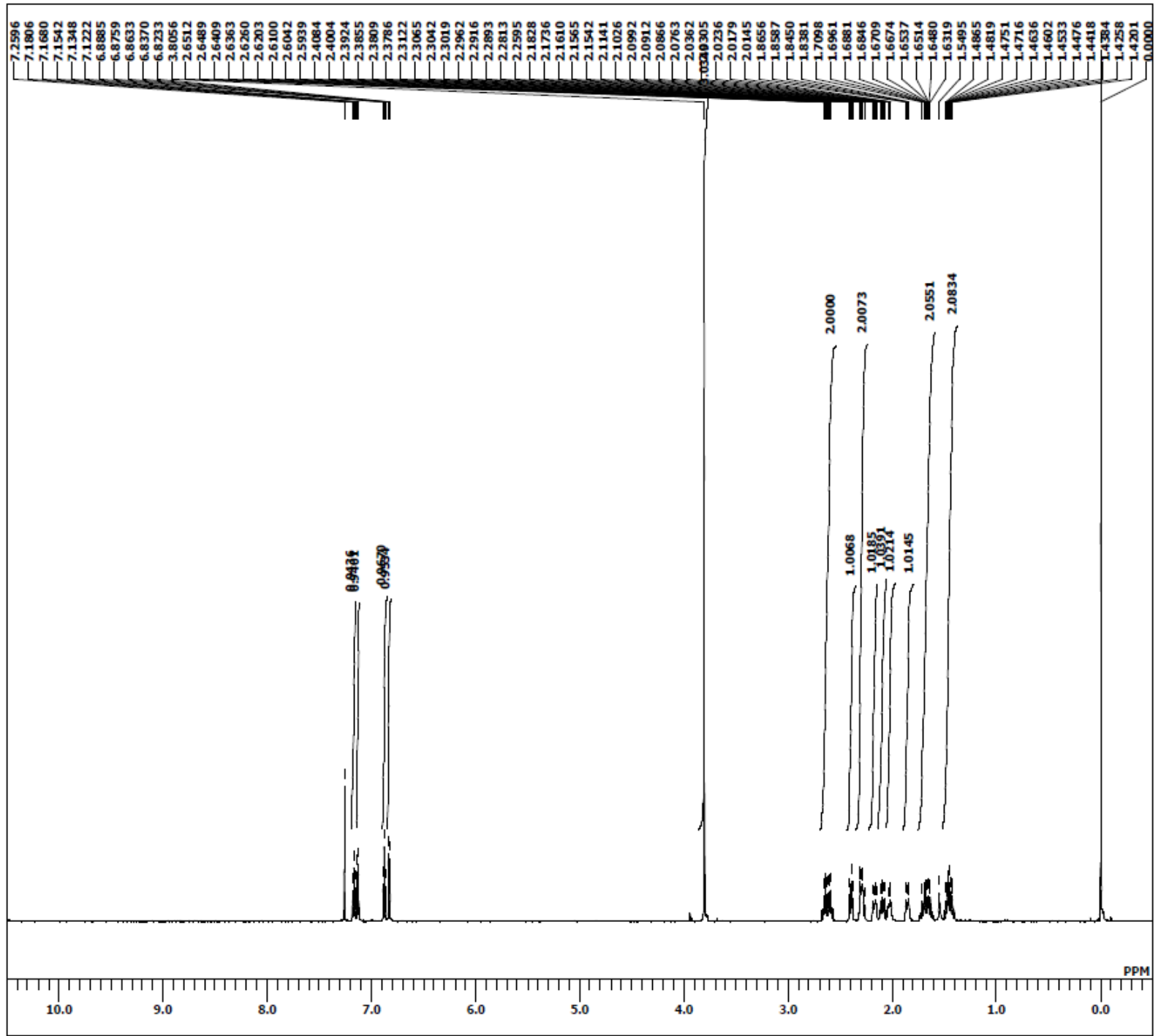


${ }^{13} \mathrm{C}$ NMR of 3 ma $\left(150 \mathrm{MHz}, \mathrm{CDCl}_{3}\right)$

疗

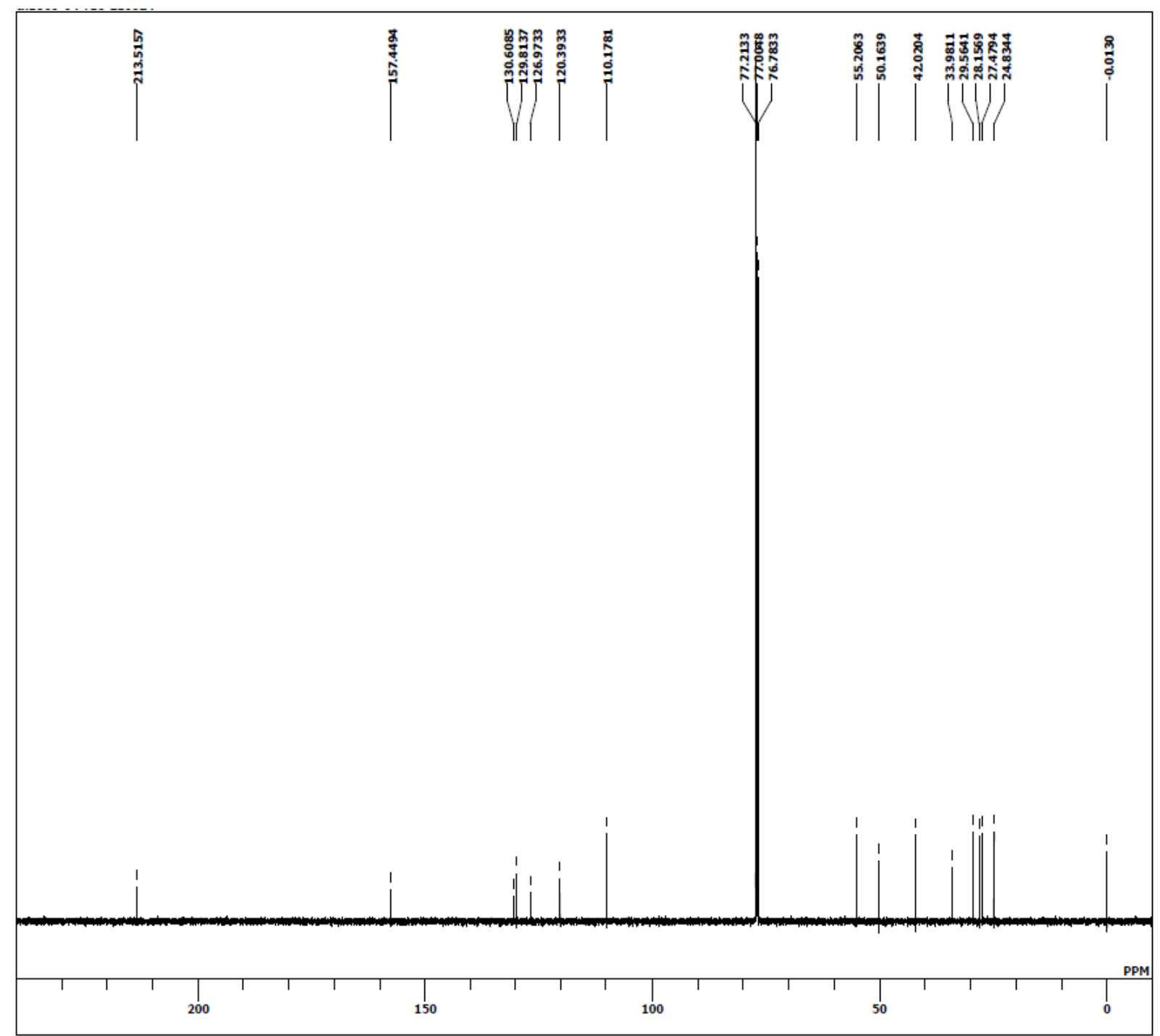


${ }^{1} \mathrm{H}$ NMR of 3na $\left(600 \mathrm{MHz}, \mathrm{CDCl}_{3}\right)$<smiles>COc1ccccc1CCC1CCCCC1=O</smiles>

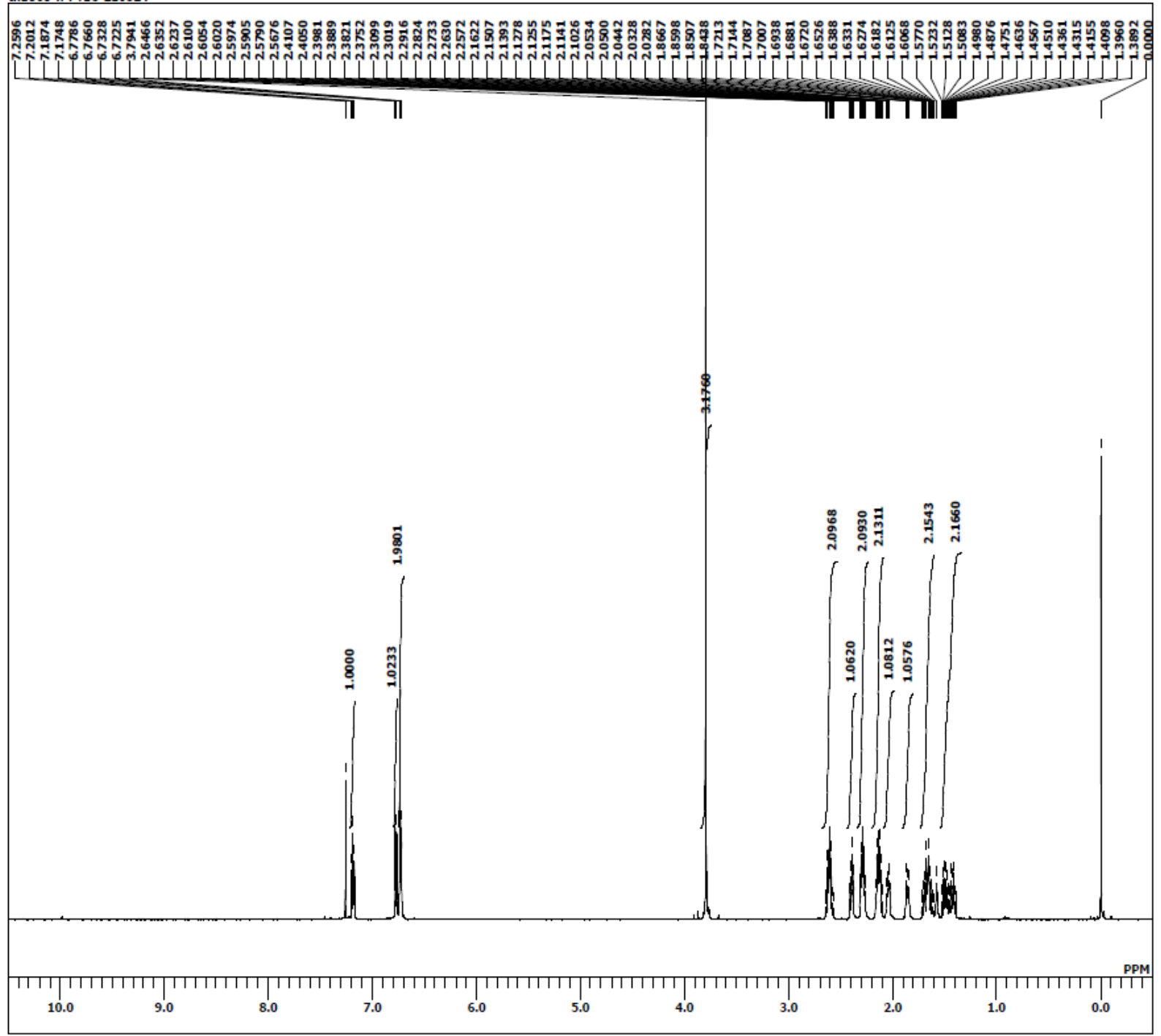


${ }^{13} \mathrm{C}$ NMR of 3na $\left(150 \mathrm{MHz}, \mathrm{CDCl}_{3}\right)$

OMe

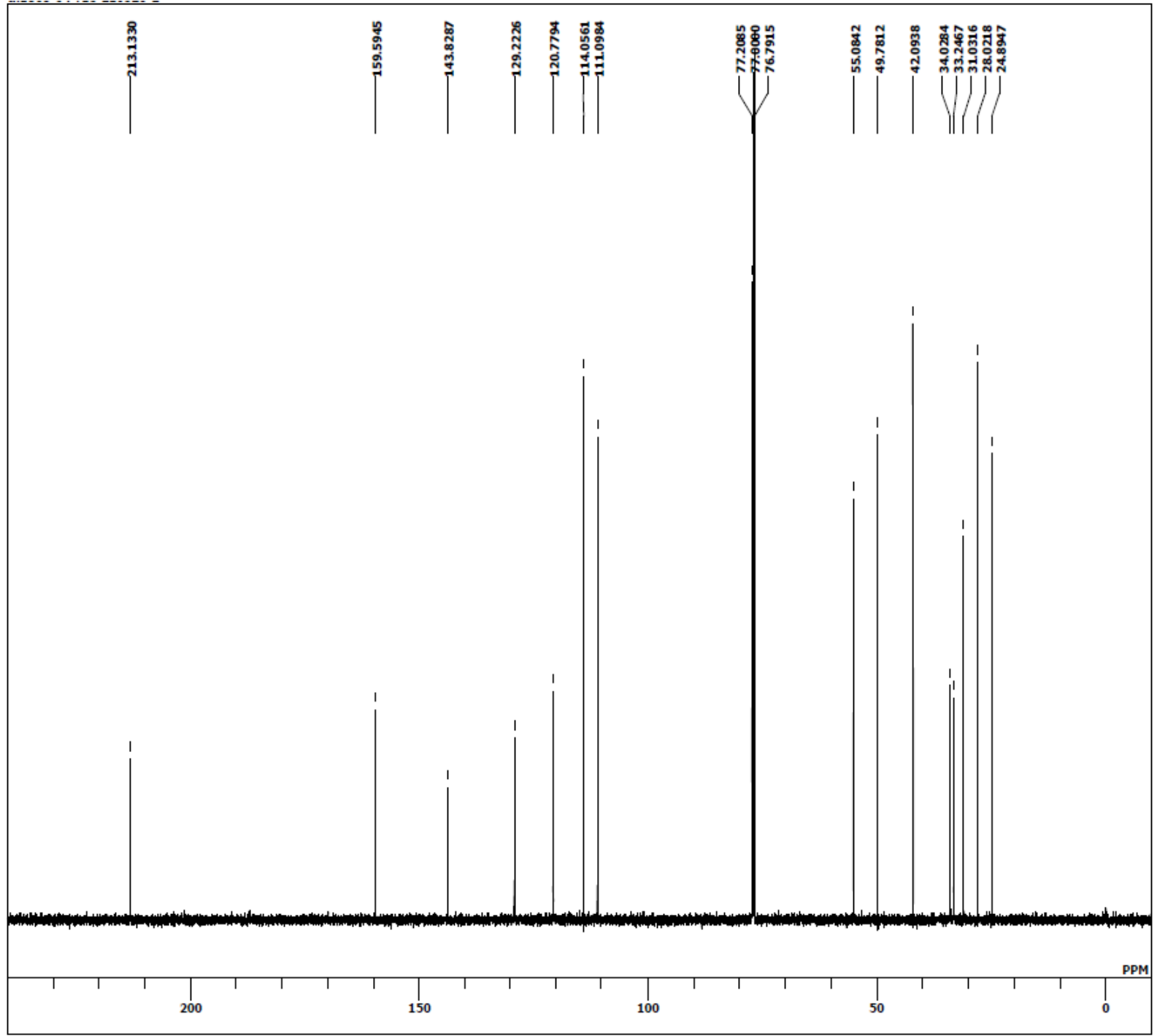


${ }^{1} \mathrm{H}$ NMR of $3 \mathbf{o a}\left(600 \mathrm{MHz}, \mathrm{CDCl}_{3}\right)$

$\mathrm{Cl}$<smiles>O=C1CCCCC1CCc1ccccc1</smiles>




${ }^{13} \mathrm{C}$ NMR of $3 o a\left(150 \mathrm{MHz}, \mathrm{CDCl}_{3}\right)$

$\mathrm{Cl}$<smiles>O=C1CCCCC1CCc1ccccc1</smiles>




${ }^{1} \mathrm{H}$ NMR of 3pa $\left(600 \mathrm{MHz}, \mathrm{CDCl}_{3}\right)$

IN)

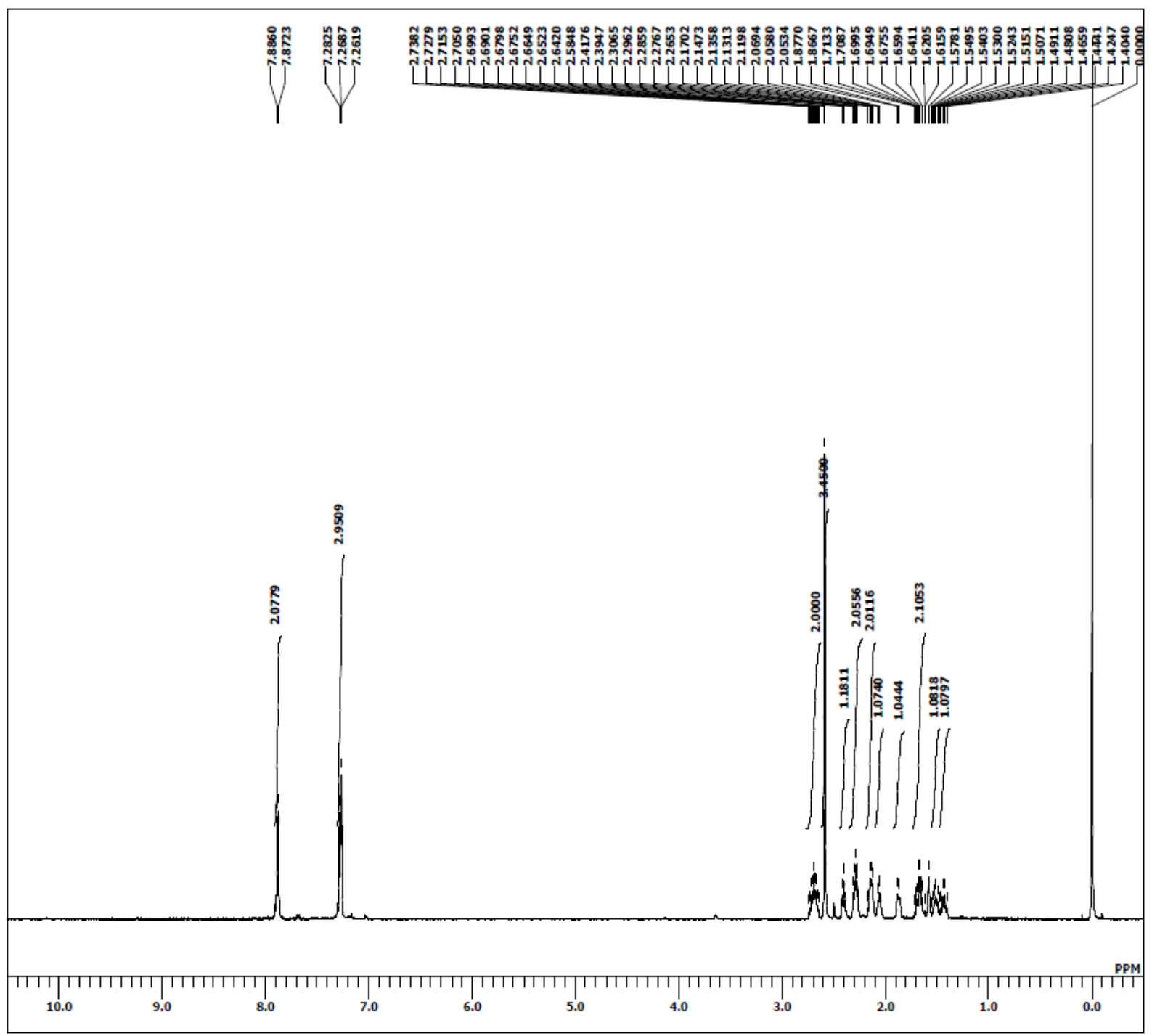


${ }^{13} \mathrm{C}$ NMR of 3pa $\left(150 \mathrm{MHz}, \mathrm{CDCl}_{3}\right)$

管

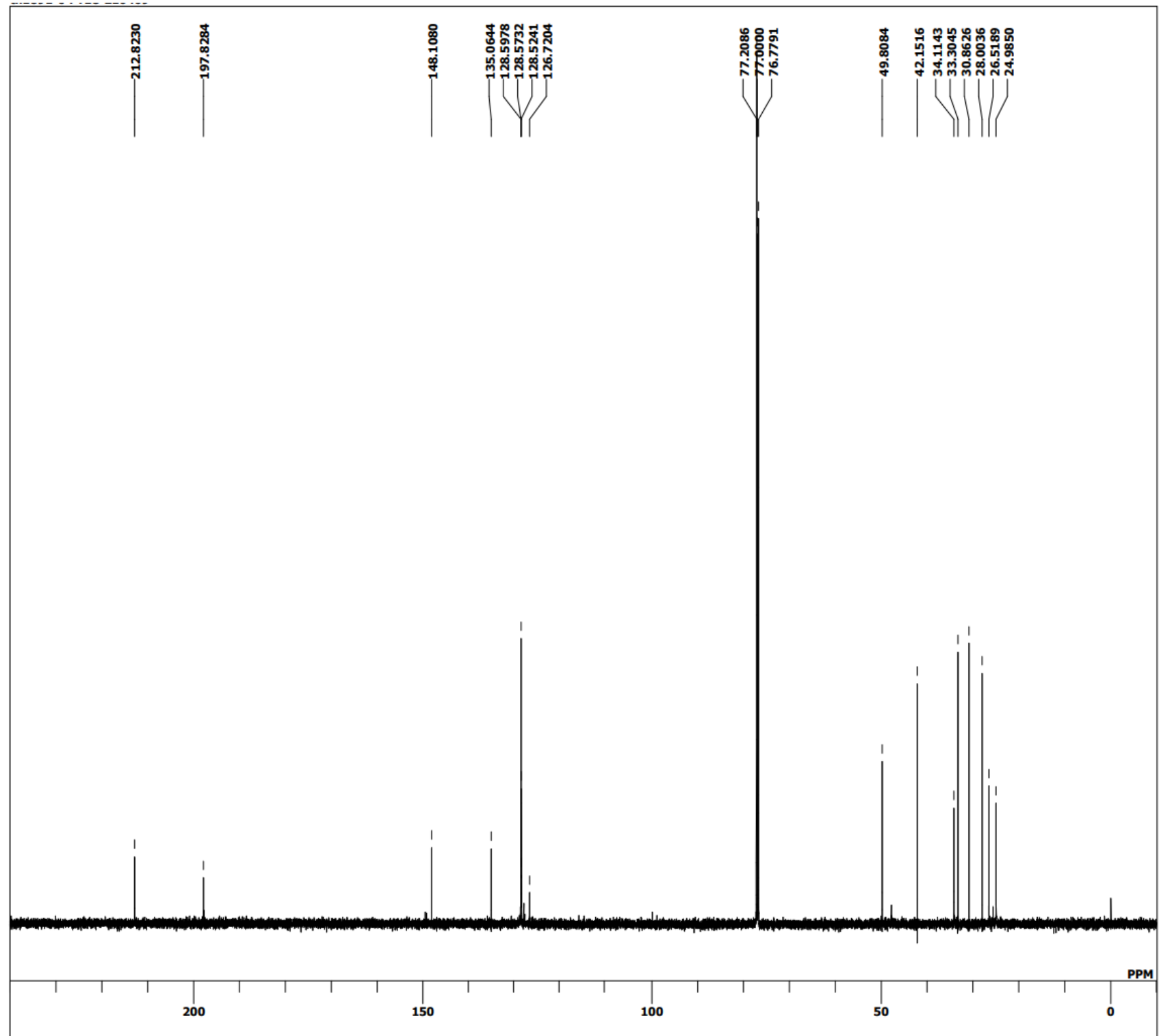


${ }^{1} \mathrm{H} \mathrm{NMR}$ of $3 q \mathbf{a}\left(600 \mathrm{MHz}, \mathrm{CDCl}_{3}\right)$

NC<smiles>Cc1ccc(CCC2CCCCC2=O)cc1</smiles>

稳管

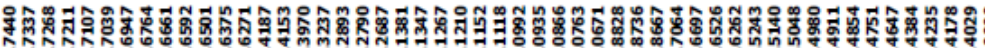

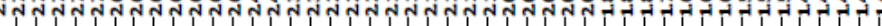

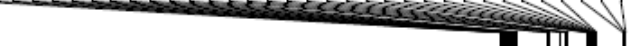

III

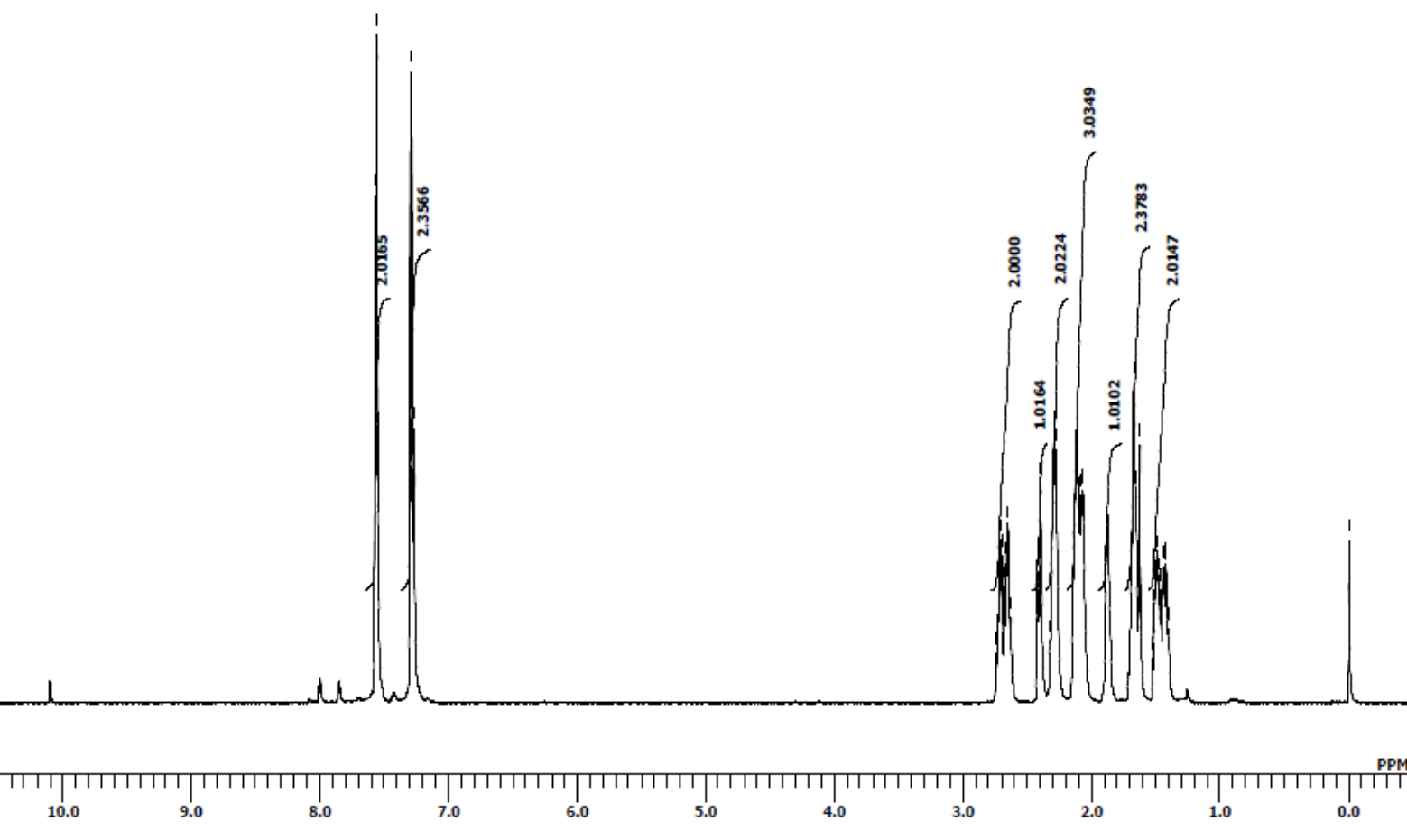


${ }^{13} \mathrm{C}$ NMR of 3qa $\left(150 \mathrm{MHz}, \mathrm{CDCl}_{3}\right)$

NC<smiles>Cc1ccc(CCC2CCCCC2=O)cc1</smiles>

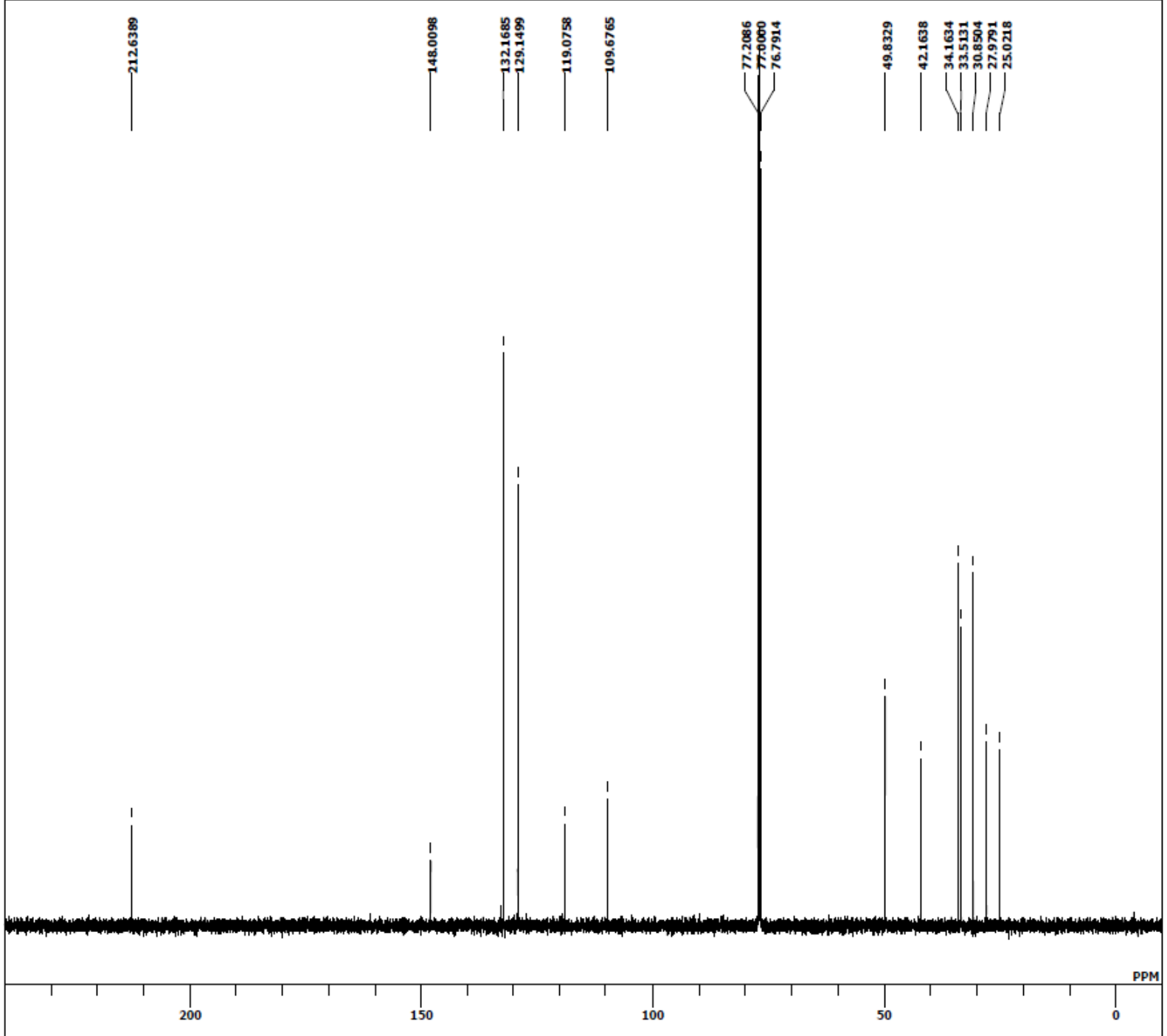


${ }^{1} \mathrm{H}$ NMR of 3ra $\left(600 \mathrm{MHz}, \mathrm{CDCl}_{3}\right)$<smiles>O=C1CCCCC1CCc1ccc2ccccc2c1</smiles>

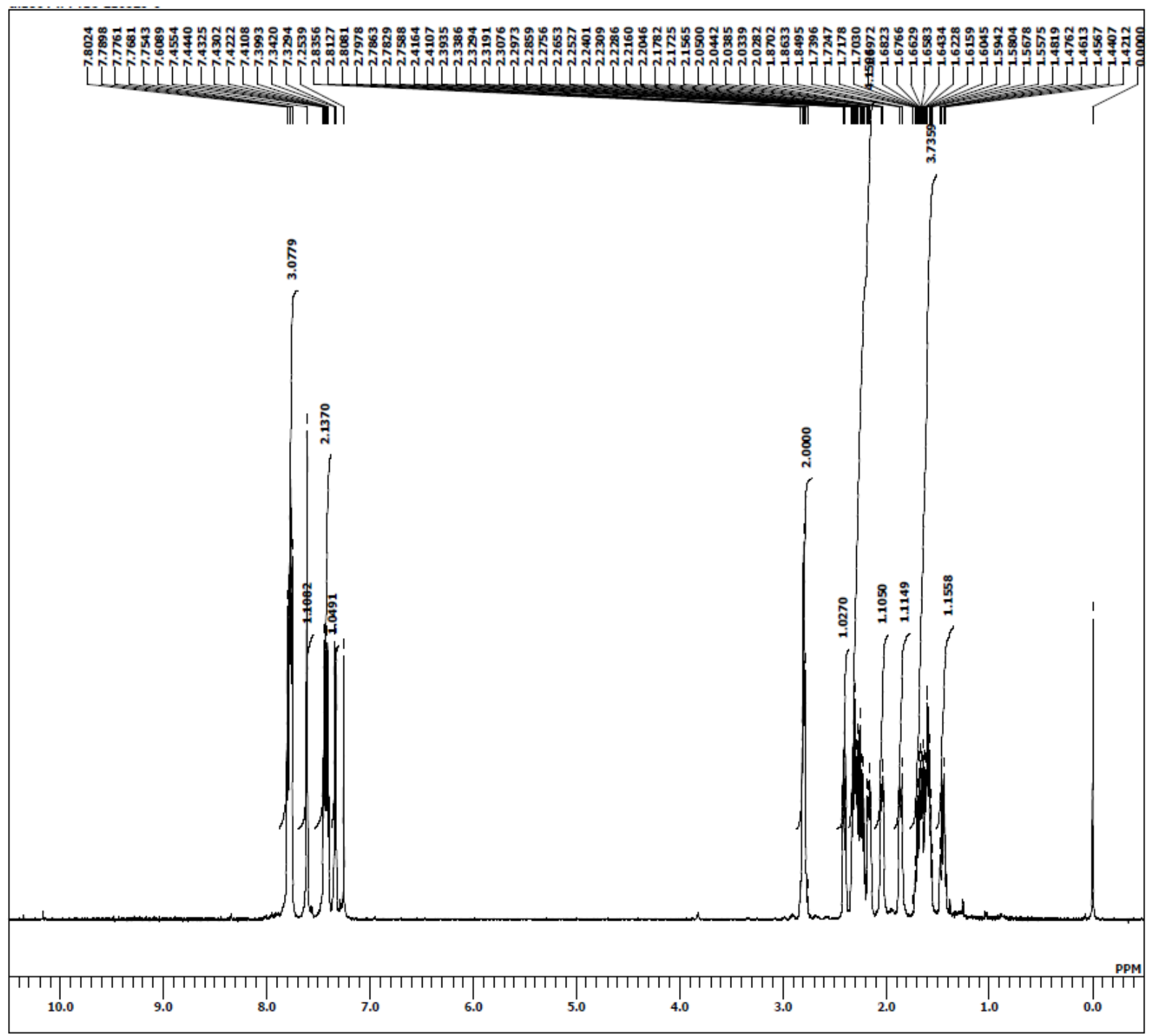


${ }^{13} \mathrm{C}$ NMR of 3ra $\left(150 \mathrm{MHz}, \mathrm{CDCl}_{3}\right)$

${ }^{1} \mathrm{H}$ NMR of 3sa $\left(600 \mathrm{MHz}, \mathrm{CDCl}_{3}\right)$
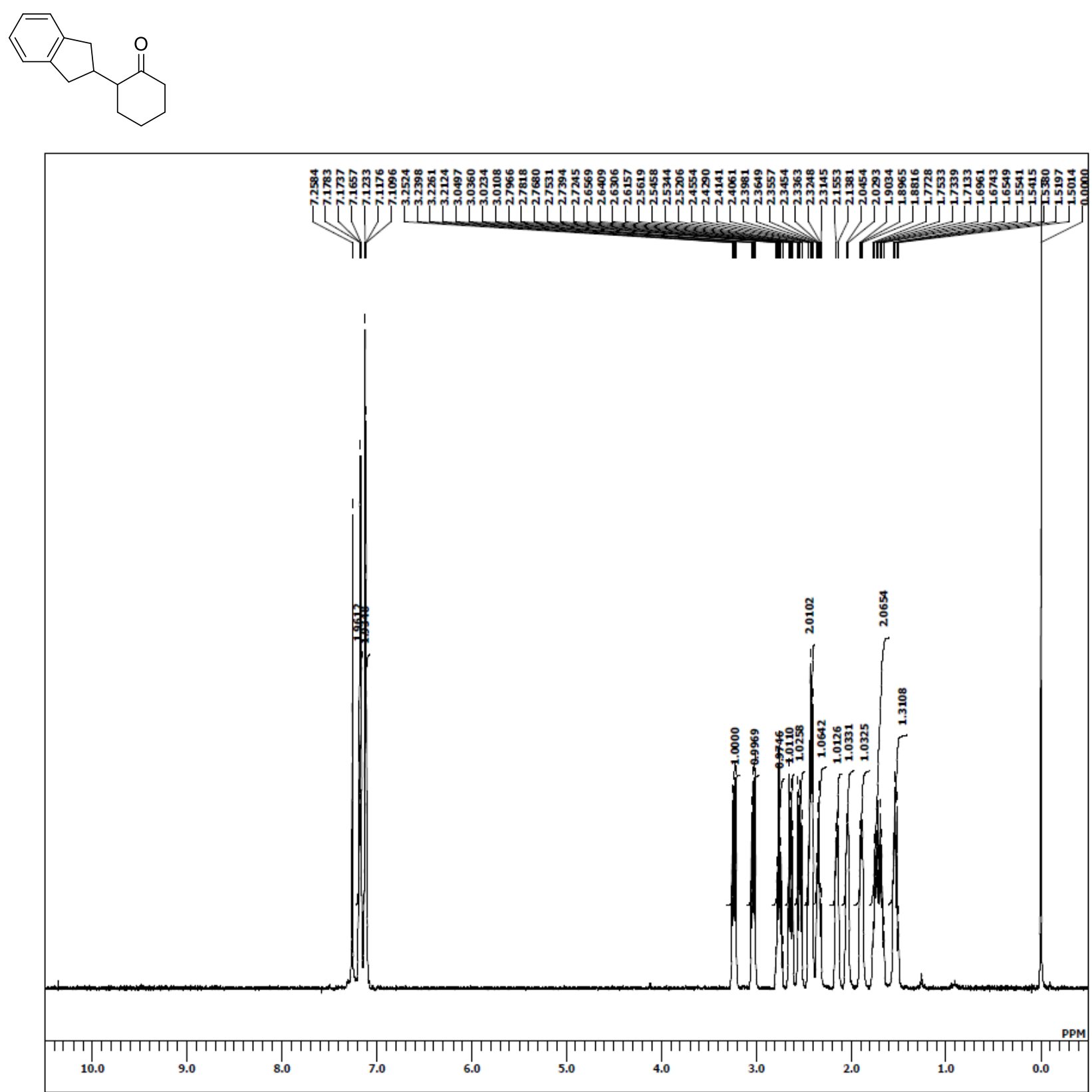
${ }^{13} \mathrm{C}$ NMR of 3sa $\left(150 \mathrm{MHz}, \mathrm{CDCl}_{3}\right)$

Dos

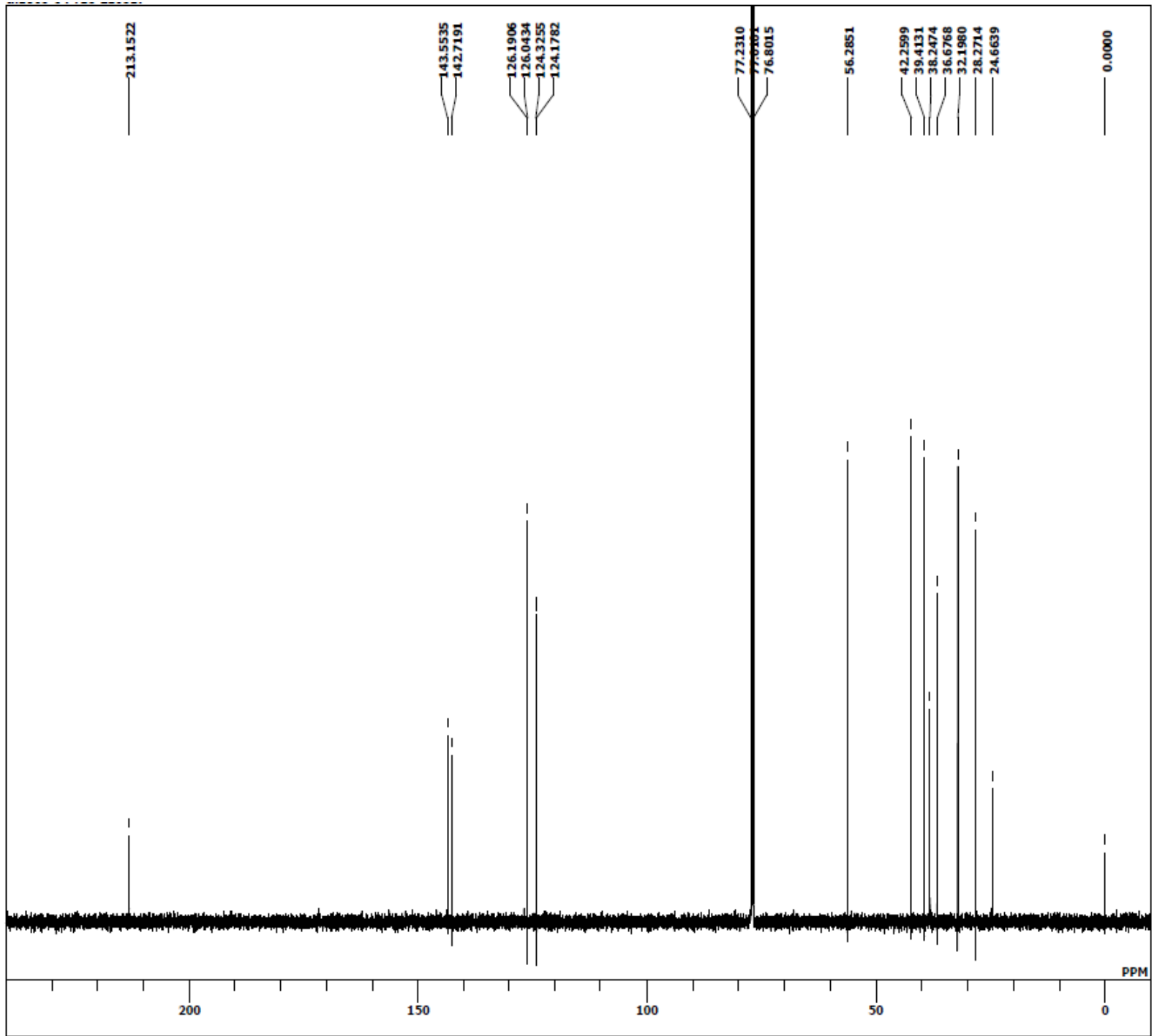


${ }^{1} \mathrm{H}$ NMR of 3 ta $\left(600 \mathrm{MHz}, \mathrm{CDCl}_{3}\right)$
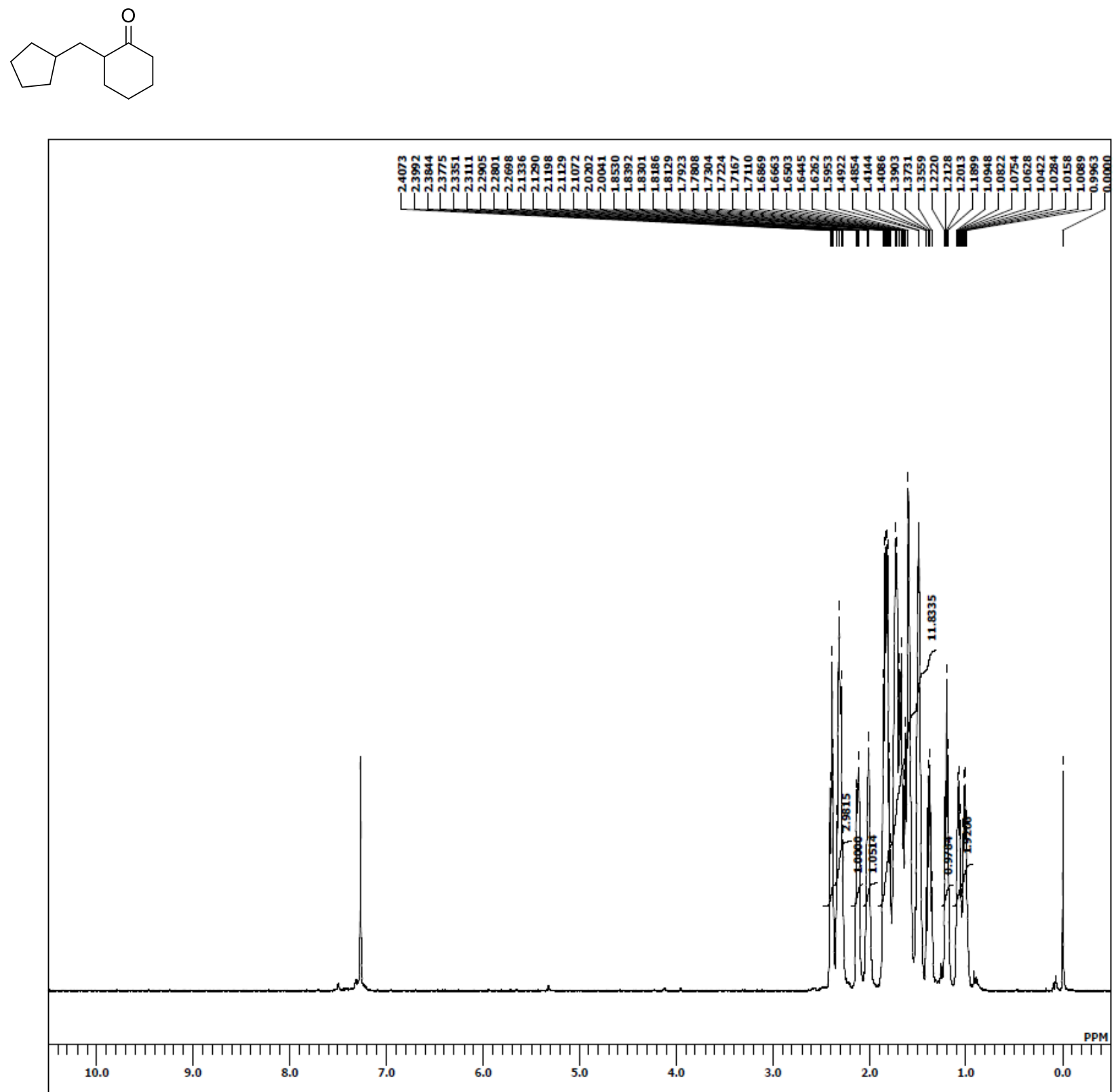
${ }^{13} \mathrm{C}$ NMR of 3ta $\left(150 \mathrm{MHz}, \mathrm{CDCl}_{3}\right)$

$\checkmark 1$

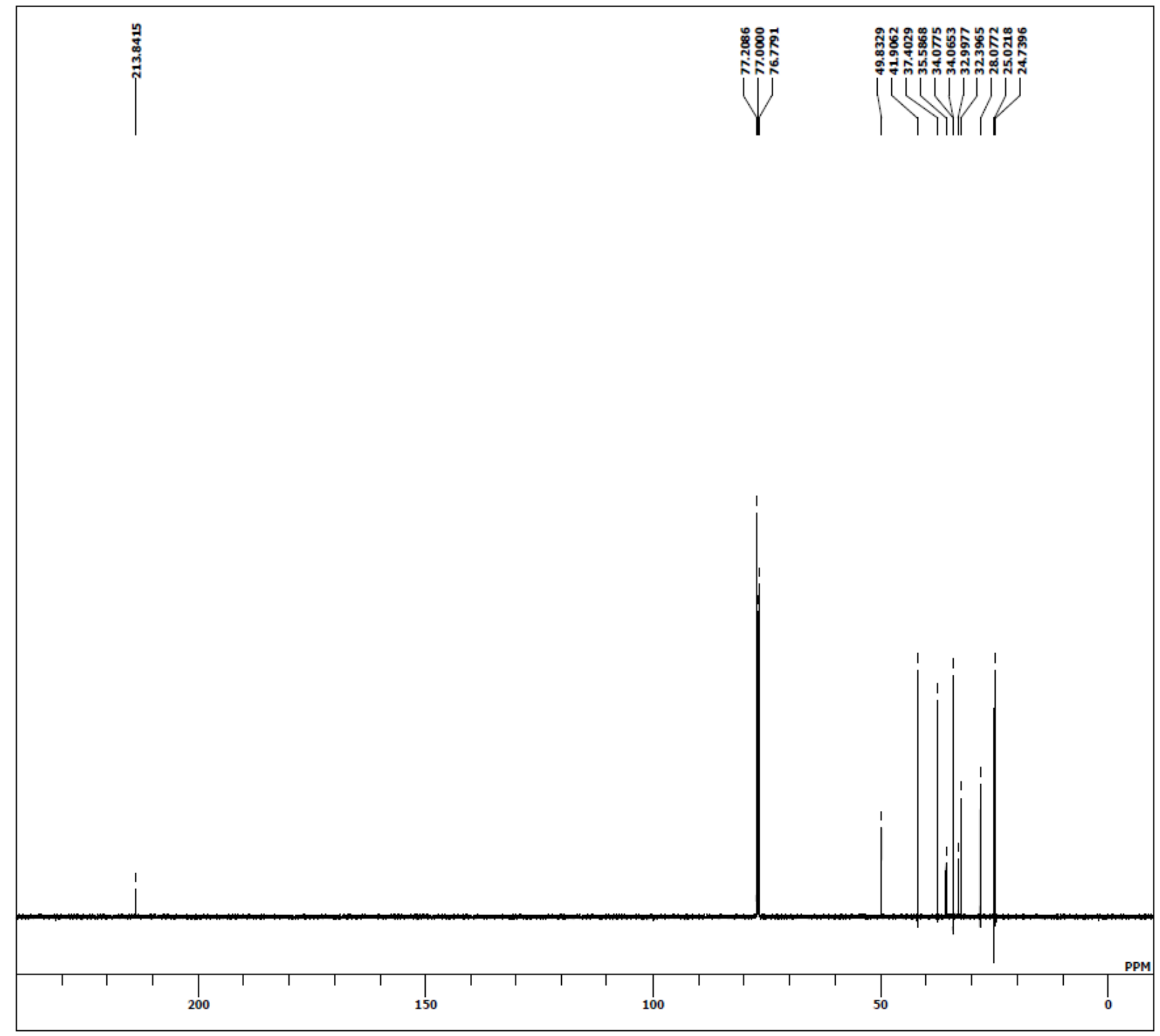


${ }^{1} \mathrm{H}$ NMR of 3 ua $\left(600 \mathrm{MHz}, \mathrm{CDCl}_{3}\right)$<smiles>O=C1CCCCC1CC1CCCCC1</smiles>

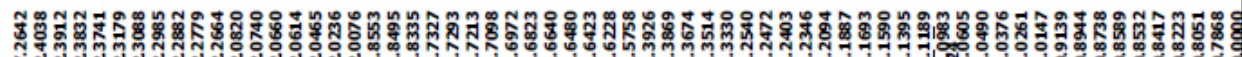
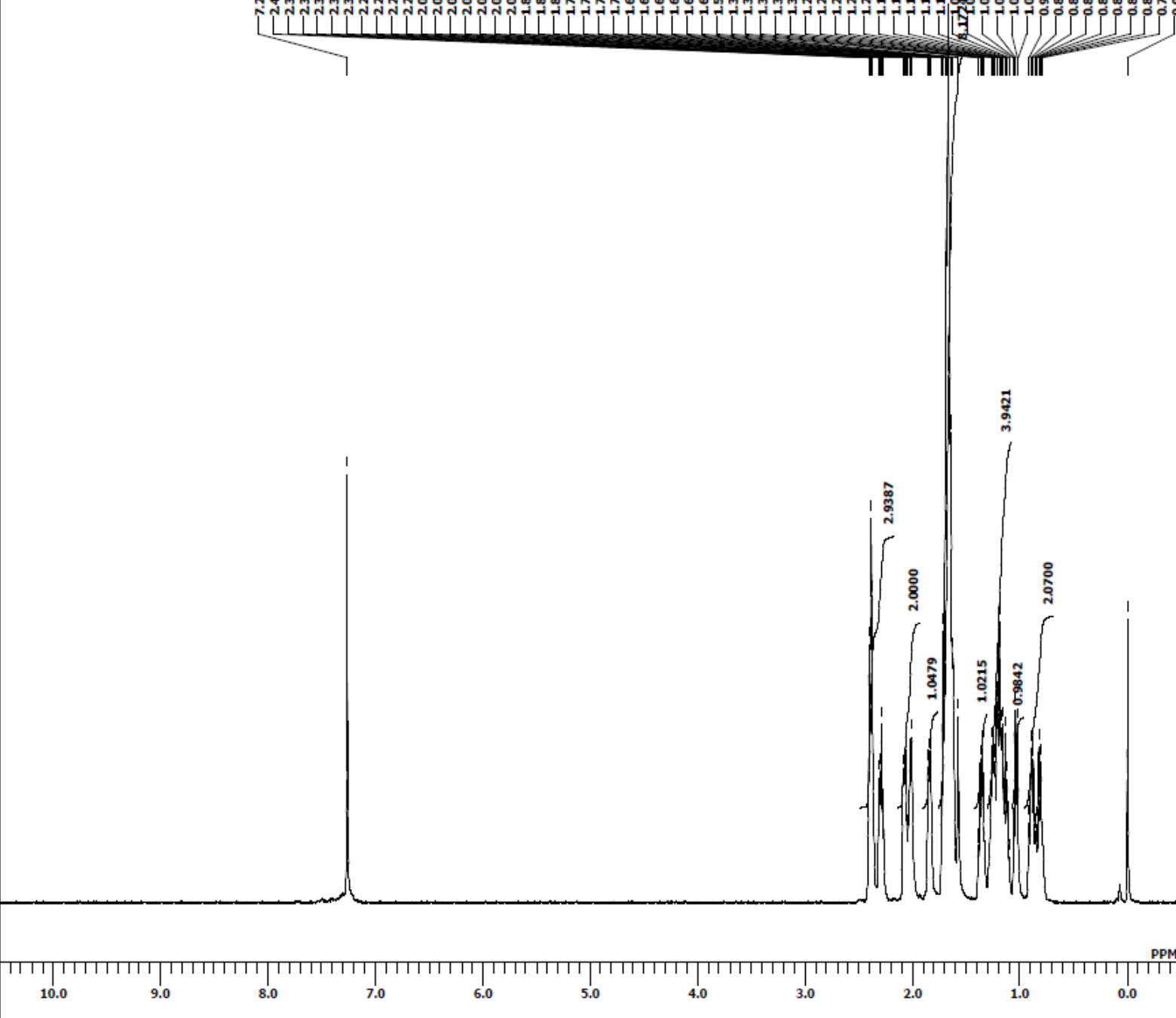
${ }^{13} \mathrm{C}$ NMR of 3 ua $\left(150 \mathrm{MHz}, \mathrm{CDCl}_{3}\right)$<smiles>O=C1CCCCC1CC1CCCCC1</smiles>

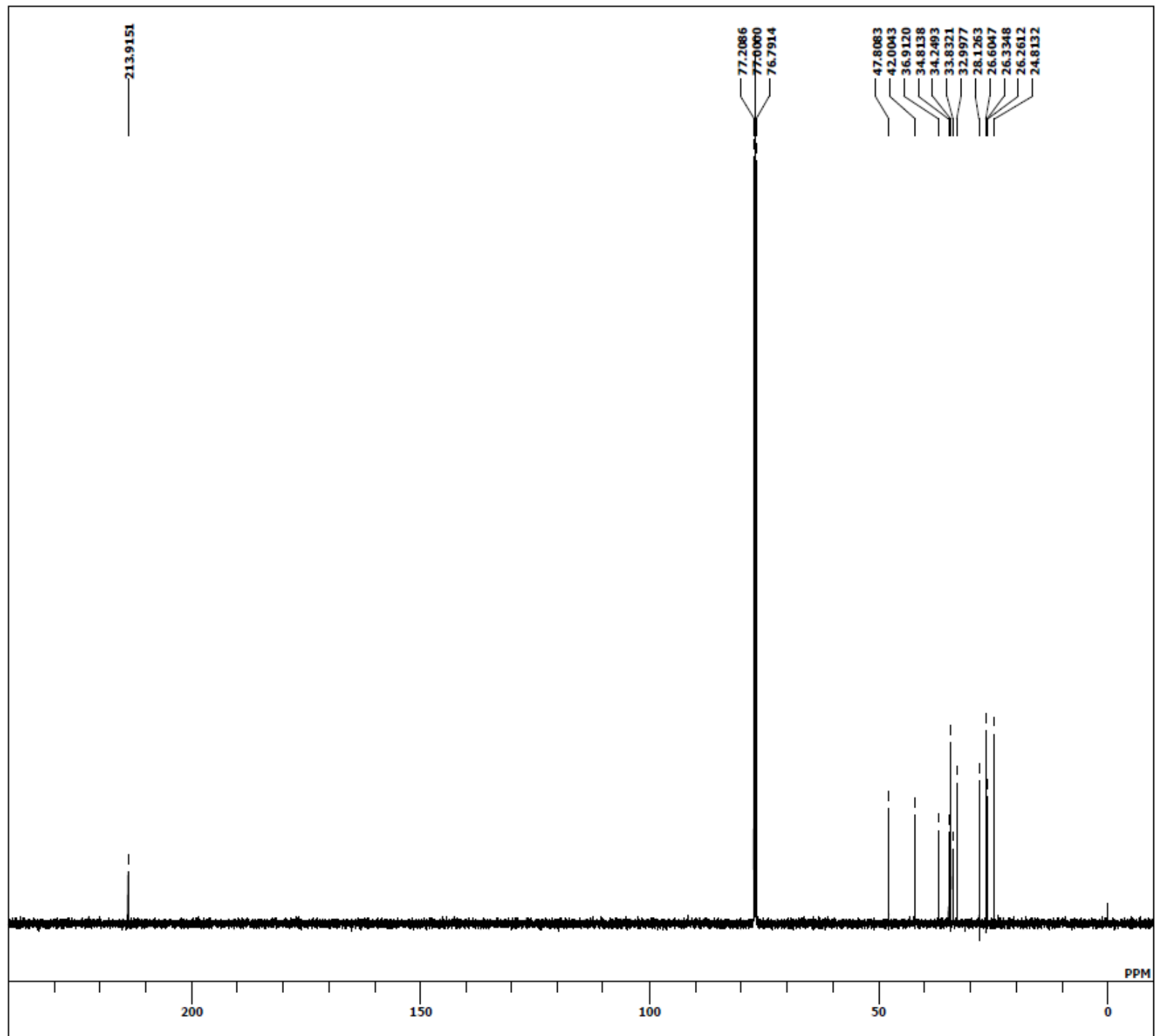


${ }^{1} \mathrm{H}$ NMR of $\mathbf{3 a b}\left(600 \mathrm{MHz}, \mathrm{CDCl}_{3}\right)$

Ph

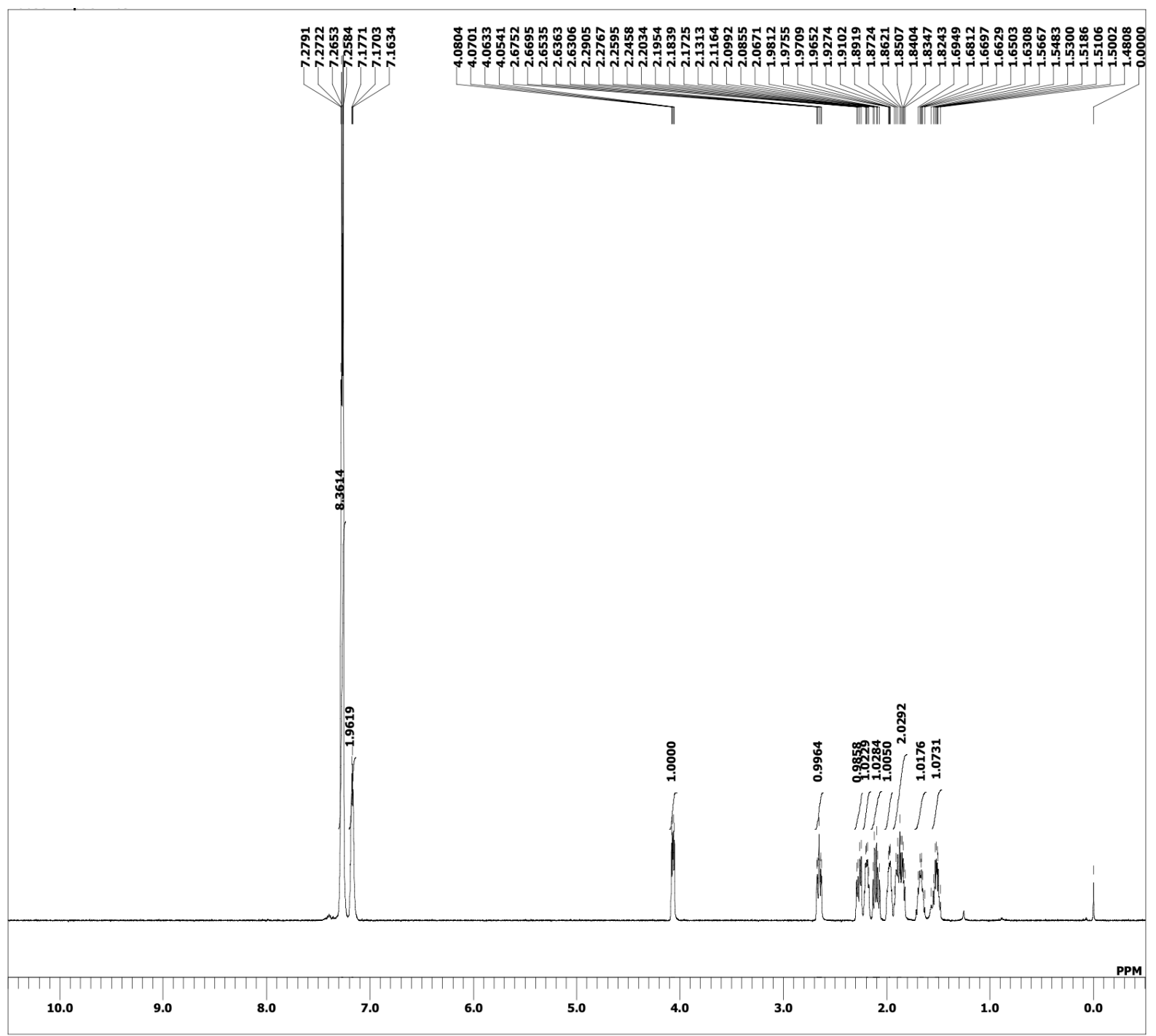


${ }^{13} \mathrm{C}$ NMR of $\mathbf{3 a b}\left(150 \mathrm{MHz}, \mathrm{CDCl}_{3}\right)$

$\mathrm{Ph}_{\mathrm{Ph}}^{\mathrm{Ph}}$

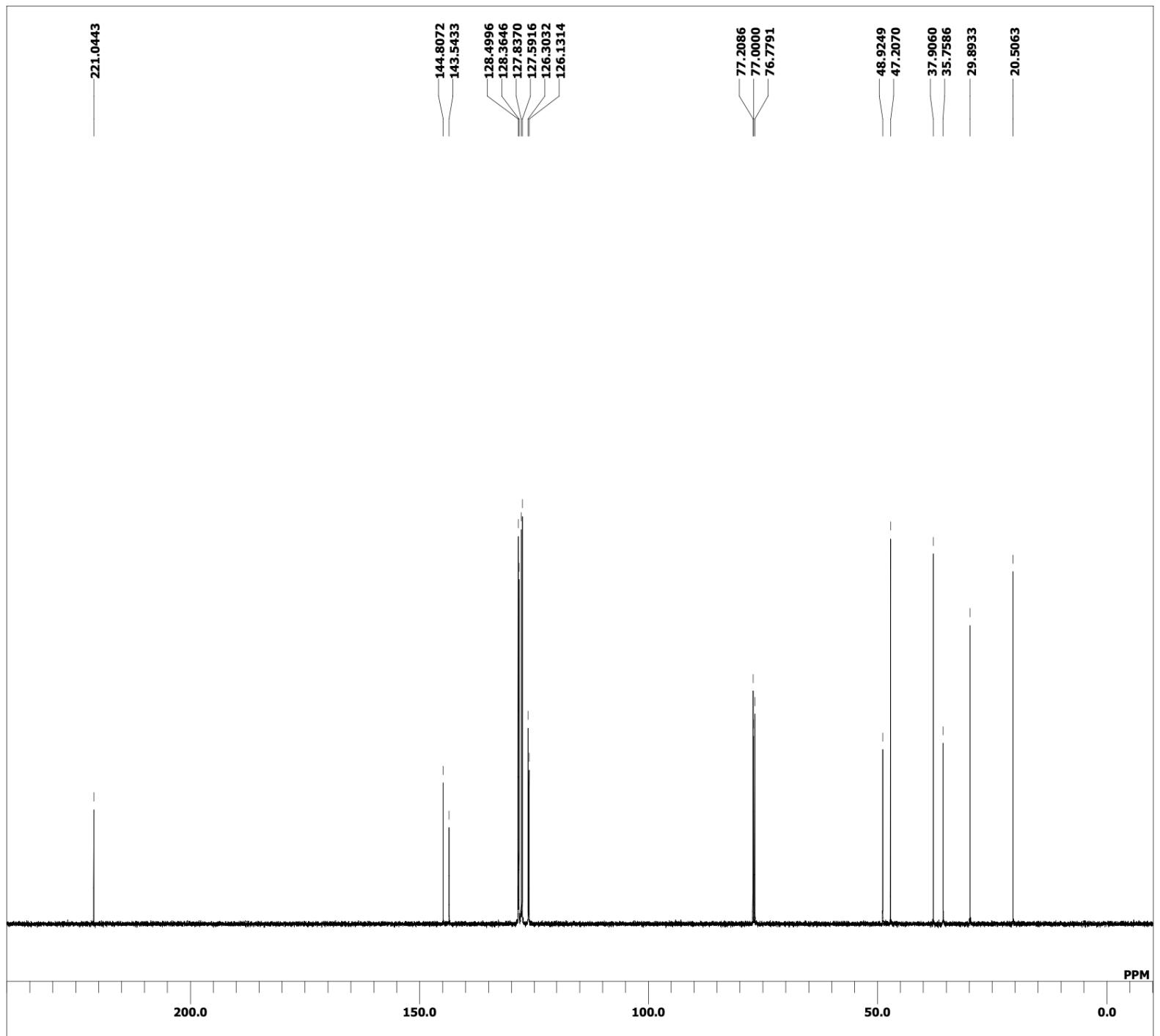


${ }^{1} \mathrm{H}$ NMR of $3 \mathbf{a c}\left(600 \mathrm{MHz}, \mathrm{CDCl}_{3}\right)$<smiles>O=C1CCCCCC1CC(c1ccccc1)c1ccccc1</smiles>

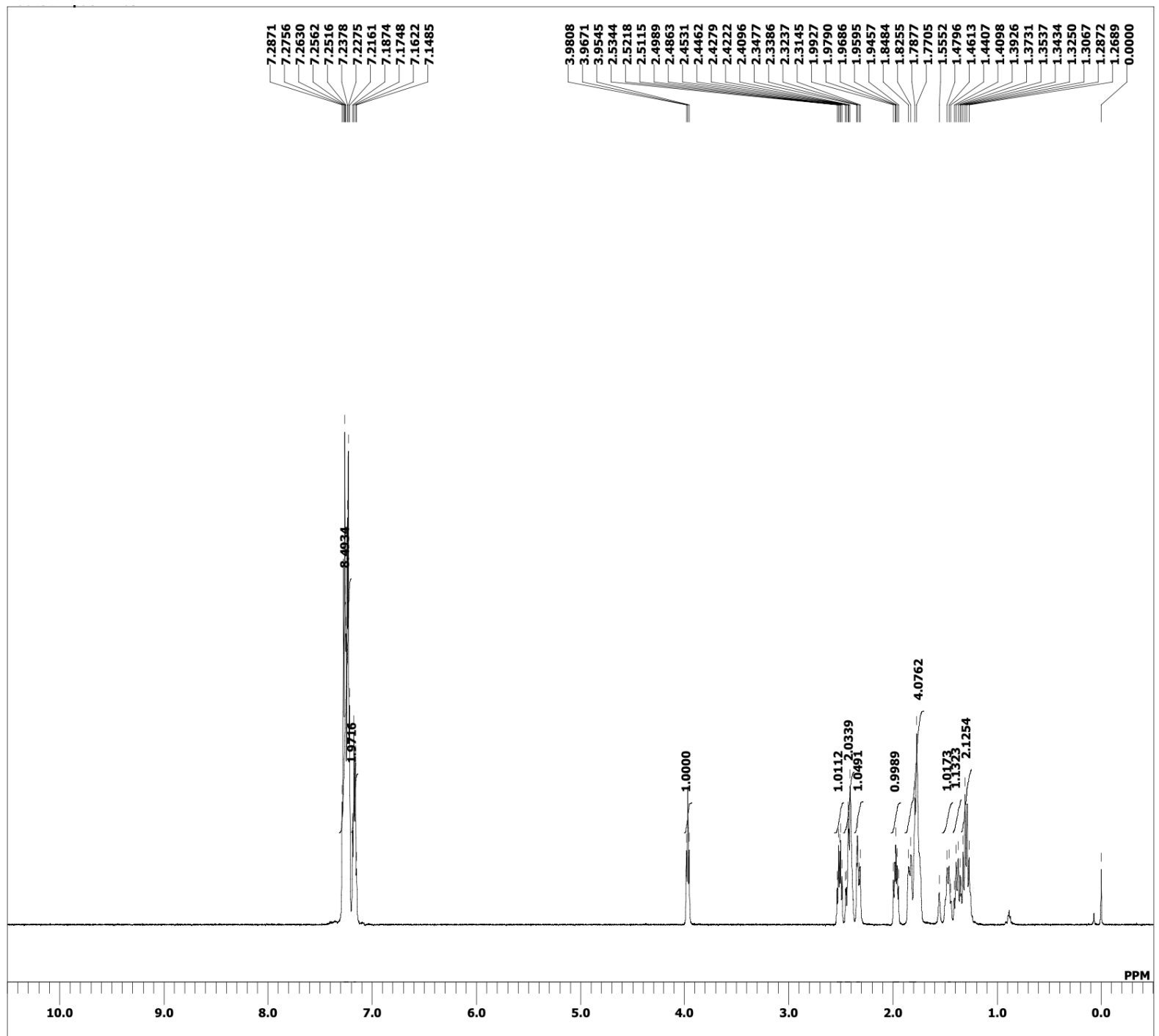


${ }^{13} \mathrm{C}$ NMR of $\mathbf{3 a c}\left(150 \mathrm{MHz}, \mathrm{CDCl}_{3}\right)$<smiles>O=C1CCCCC1CC(c1ccccc1)c1ccccc1</smiles>

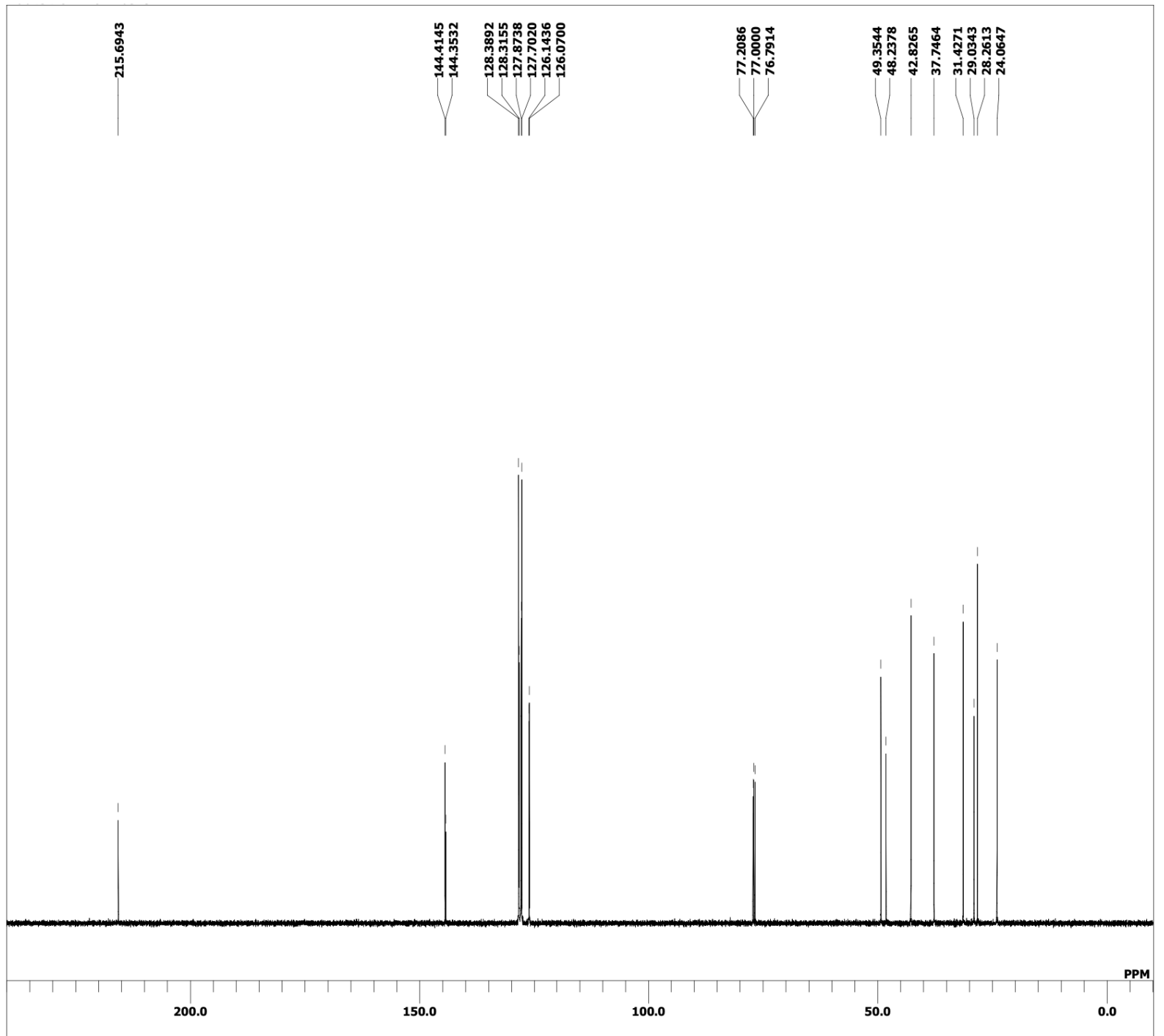


${ }^{1} \mathrm{H} \mathrm{NMR}$ of 3ad $\left(600 \mathrm{MHz}, \mathrm{CDCl}_{3}\right)$<smiles>CC1CCC(=O)C(Cc2ccccc2)C1</smiles>

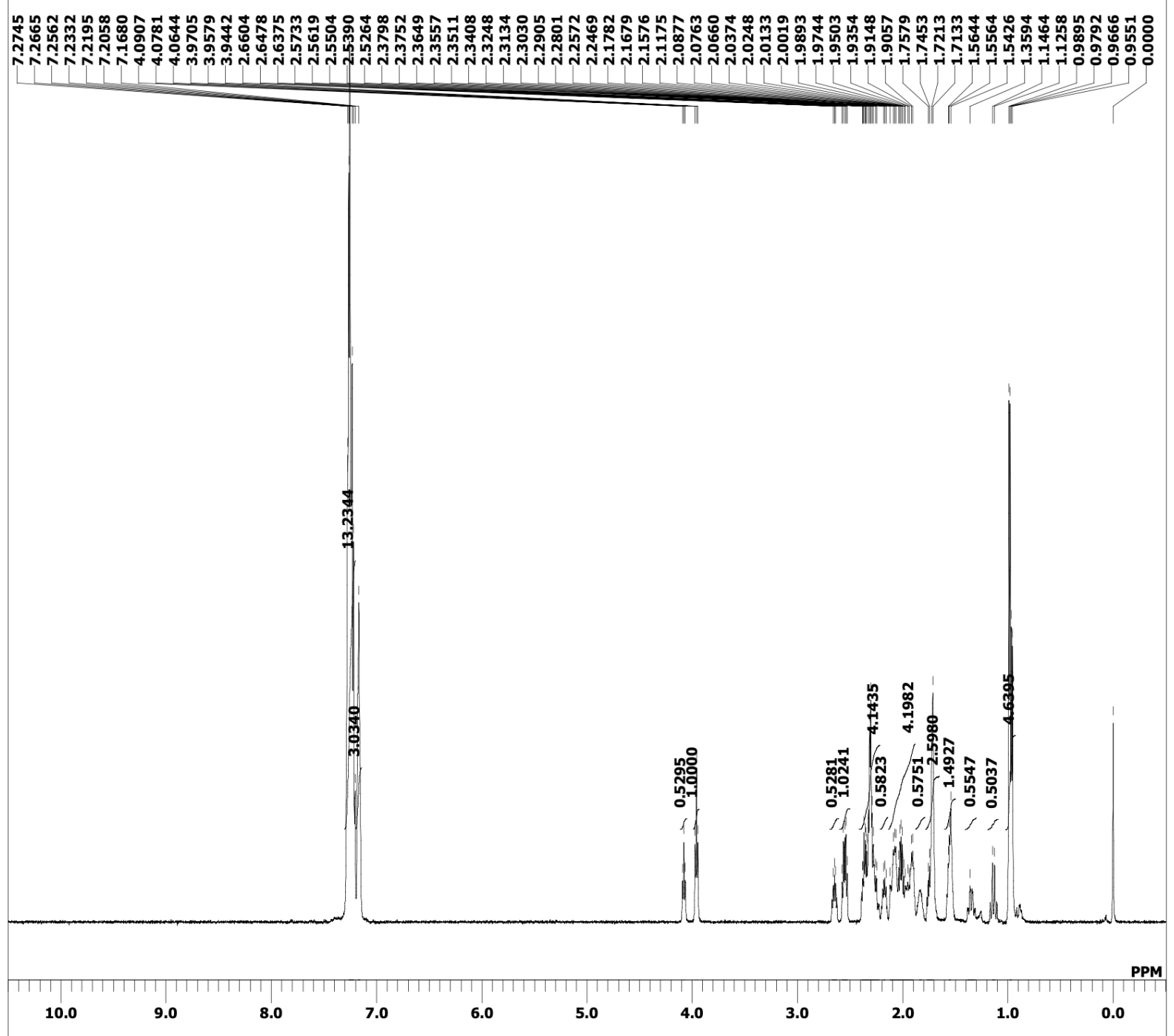


${ }^{13} \mathrm{C}$ NMR of $3 a d\left(150 \mathrm{MHz}, \mathrm{CDCl}_{3}\right)$

(1)

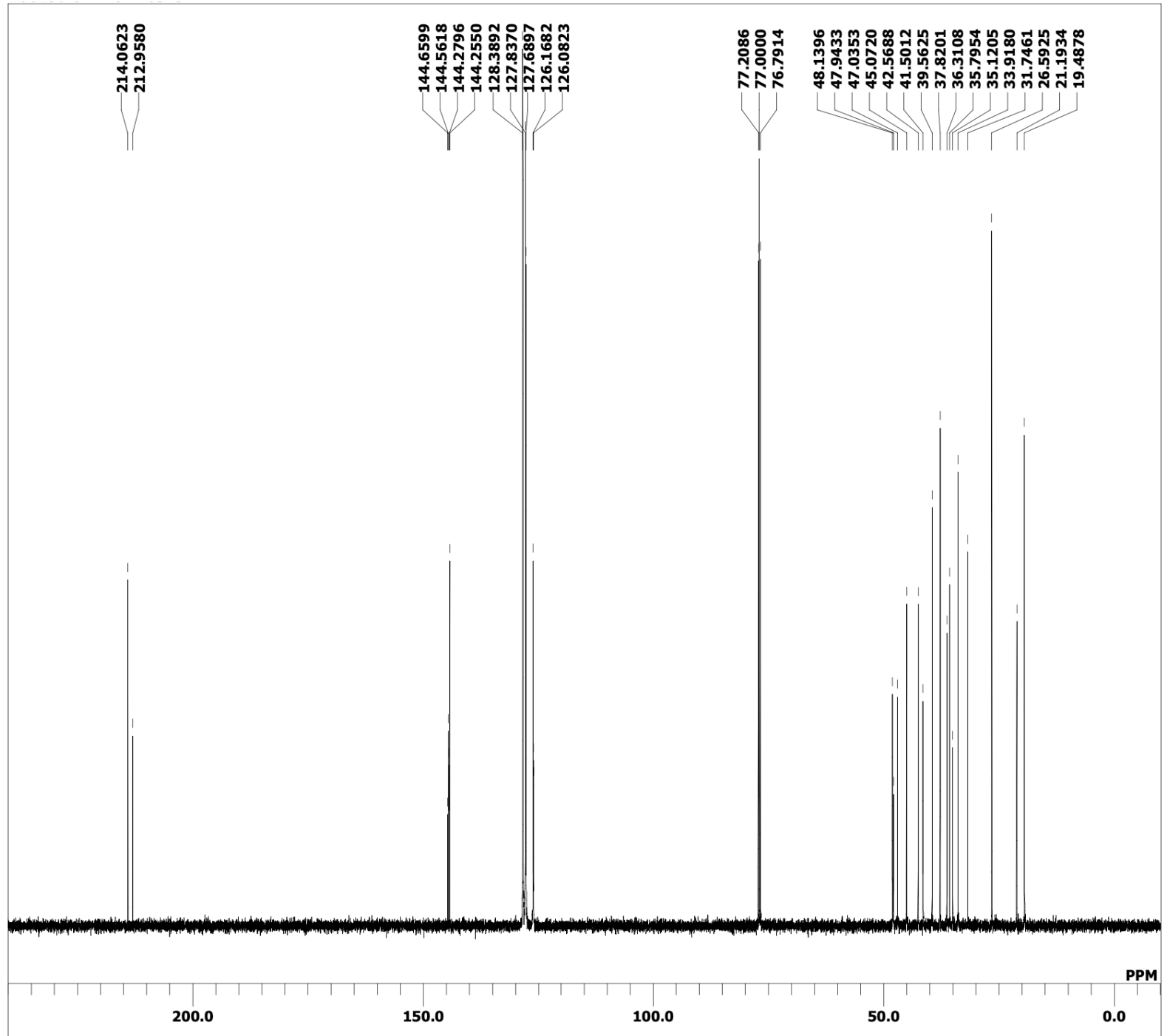


${ }^{1} \mathrm{H}$ NMR of $\mathbf{3 a e}\left(600 \mathrm{MHz}, \mathrm{CDCl}_{3}\right)$<smiles>O=C1CCC[C@H]2CC(c3ccccc3)[C@@H]3C[C@@H]1CCC23</smiles>

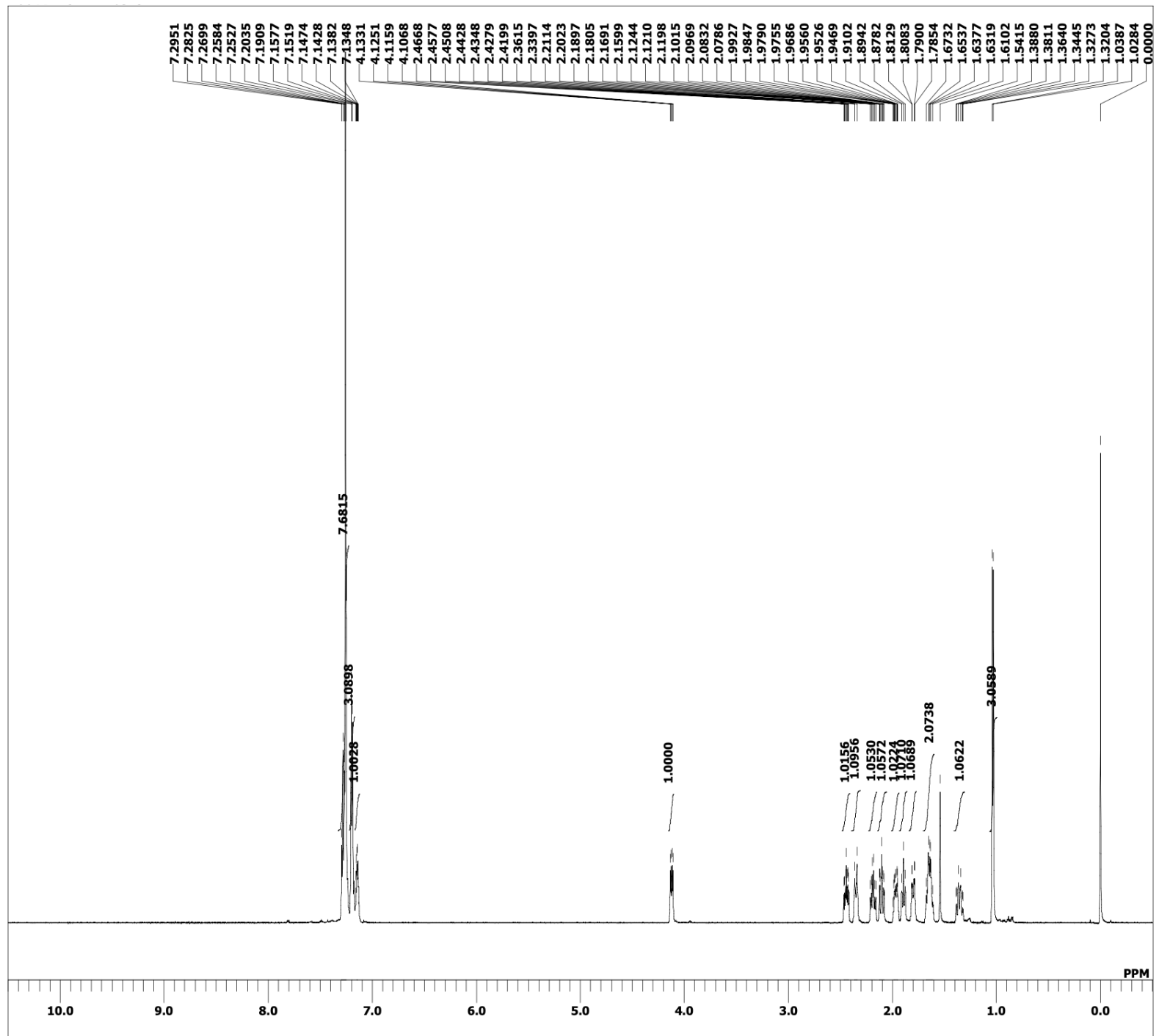


${ }^{13} \mathrm{C}$ NMR of 3ae $\left(150 \mathrm{MHz}, \mathrm{CDCl}_{3}\right)$

(N)




${ }^{1} \mathrm{H} \mathrm{NMR}$ of $\mathbf{3 a f}\left(500 \mathrm{MHz}, \mathrm{CDCl}_{3}\right)$

Ph

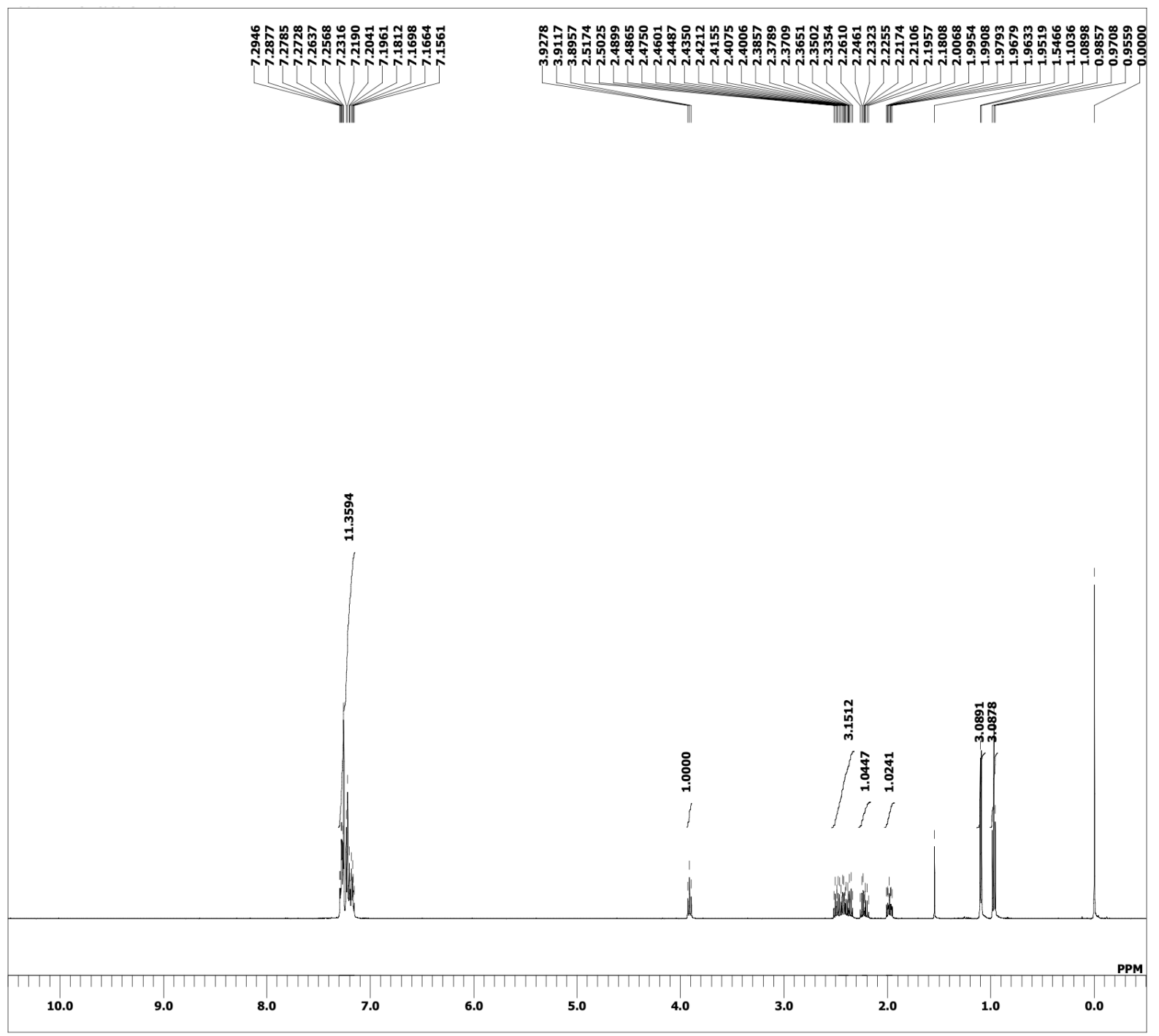


${ }^{13} \mathrm{C}$ NMR of $\mathbf{3 a f}\left(150 \mathrm{MHz}, \mathrm{CDCl}_{3}\right)$

(Ph

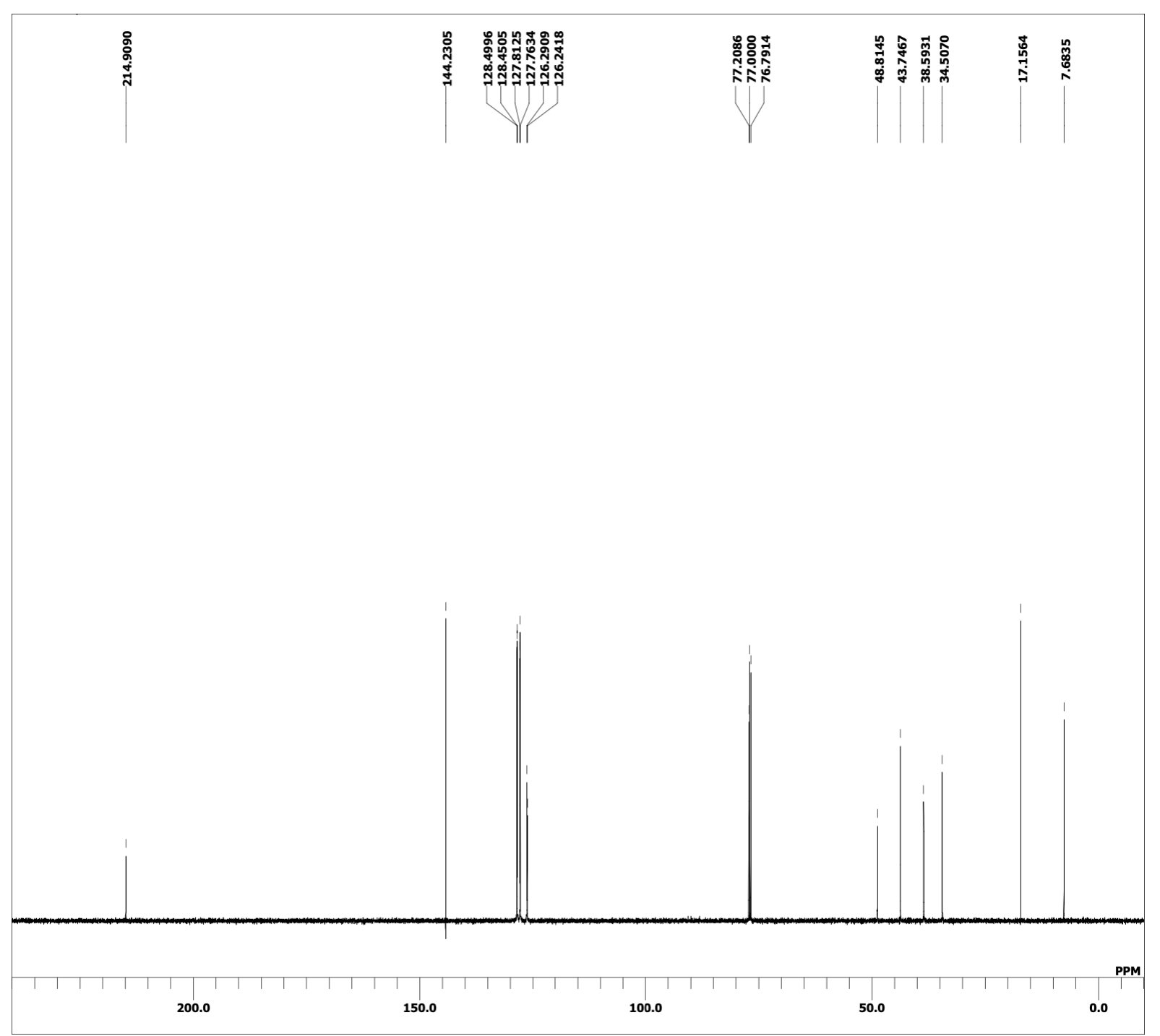




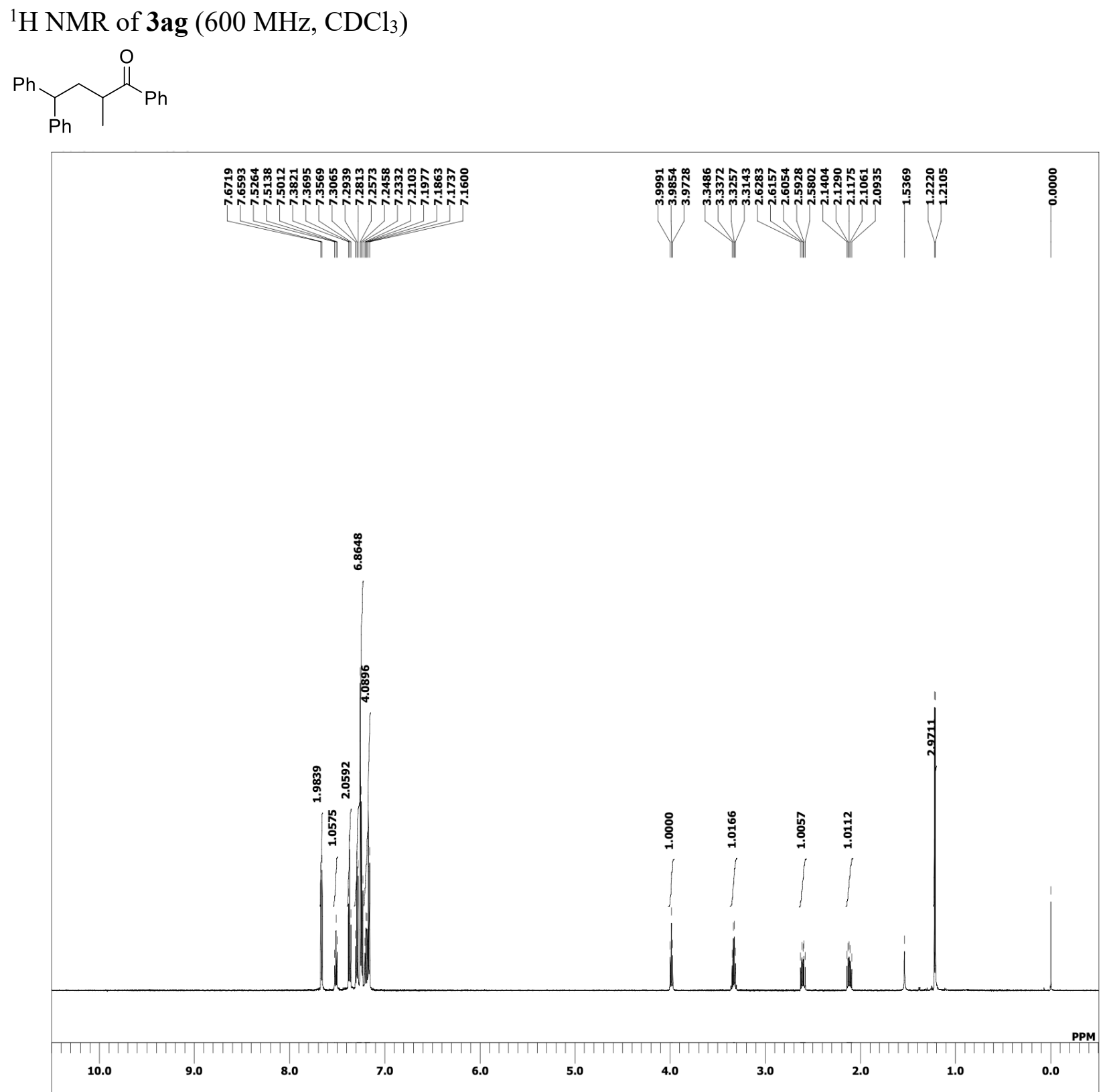


${ }^{13} \mathrm{C}$ NMR of 3 ag $\left(150 \mathrm{MHz}, \mathrm{CDCl}_{3}\right)$

${ }_{\mathrm{Ph}}^{\mathrm{O}}$

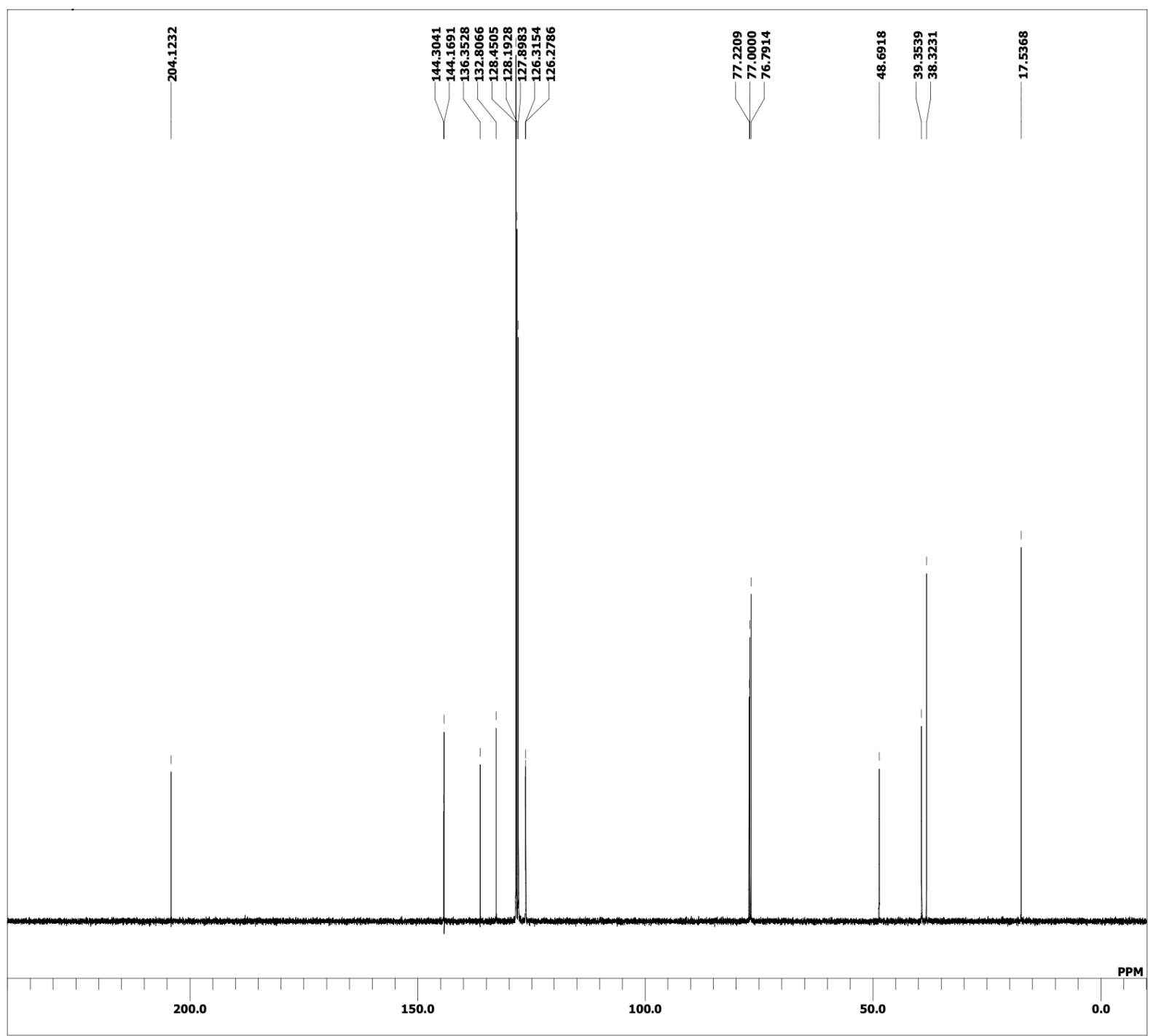

